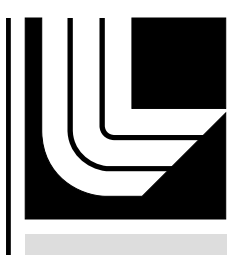

LAW RENCE LIVERMORE N A TIO N A L LABORATORY

Adjoint Error Estimation for Linear Advection

J. M. Connors, J. W. Banks, J. A. Hittinger, C. S. Woodward

March 31, 2011 
This document was prepared as an account of work sponsored by an agency of the United States government. Neither the United States government nor Lawrence Livermore National Security, LLC, nor any of their employees makes any warranty, expressed or implied, or assumes any legal liability or responsibility for the accuracy, completeness, or usefulness of any information, apparatus, product, or process disclosed, or represents that its use would not infringe privately owned rights. Reference herein to any specific commercial product, process, or service by trade name, trademark, manufacturer, or otherwise does not necessarily constitute or imply its endorsement, recommendation, or favoring by the United States government or Lawrence Livermore National Security, LLC. The views and opinions of authors expressed herein do not necessarily state or reflect those of the United States government or Lawrence Livermore National Security, LLC, and shall not be used for advertising or product endorsement purposes.

This work performed under the auspices of the U.S. Department of Energy by Lawrence Livermore National Laboratory under Contract DE-AC52-07NA27344. 


\title{
ADJOINT ERROR ESTIMATION FOR LINEAR ADVECTION
}

\author{
J. M. CONNORS, J. W. BANKS, J. A. HITTINGER, AND C. S. WOODWARD
}

Abstract. An a posteriori error formula is described when a statistical measurement of the solution to a hyperbolic conservation law in 1D is estimated by finite volume approximations. This is accomplished using adjoint error estimation. In contrast to previously studied methods, the adjoint problem is divorced from the finite volume method used to approximate the forward solution variables. An exact error formula and computable error estimate are derived based on an abstractly defined approximation of the adjoint solution. This framework allows the error to be computed to an arbitrary accuracy given a sufficiently well resolved approximation of the adjoint solution. The accuracy of the computable error estimate provably satisfies an a priori error bound for sufficiently smooth solutions of the forward and adjoint problems. The theory does not currently account for discontinuities. Computational examples are provided that show support of the theory for smooth solutions. The application to problems with discontinuities is also investigated computationally.

\section{Contents}

1. Introduction

2. The adjoint problem and error formula

2.1. Identification of an adjoint problem for the error

2.2. The error representation formula

3. Application to constant-coefficient linear advection

3.1. Reconstruction mappings for finite volume data

3.2. Asymptotic properties of the computable error estimate

4. Computational examples

4.1. Fourier coefficient estimation

4.2. Evaluation of the solution at a point

4.3. Solution moment of order zero

4.4. Solution moment of order one

4.5. Solution moment of order two

4.6. Solution energy

4.7. Solution average value over a patch

5. Conclusions and future work

References

Date: April 7, 2011. 


\section{INTRODUCTION}

The problem is to calculate directly the error in an estimate of $\mathcal{M}(u)$, where $u=u(x, t)$ solves

$$
\begin{aligned}
\partial_{t} u+\partial_{x} f(u) & =F(x, t),(x, t) \in \Omega_{T} \\
u(x, 0) & =u_{0}(x), x \in \Omega=\left(x_{L}, x_{R}\right),
\end{aligned}
$$

with periodic boundary conditions in space, and $\mathcal{M}(u)$ is a quantity of interest. Here $\Omega_{T}=\Omega \times(0, T]$. The quantity of interest is typically some statistical measurement of the solution. We assume the quantity of interest may be expressed in one of these two forms:

$$
\begin{aligned}
\mathcal{M}(u) & =\int_{\Omega_{T}} \psi(x, t) u(x, t) d x d t \\
\text { or } \mathcal{M}(u) & =\int_{\Omega} \psi(x) u(x, T) d x .
\end{aligned}
$$

These functionals are considered to be linear with respect to $u$ for simplicity. The theory in this report can be extended easily to the nonlinear case following the standard approach outlined in (e.g.) [6]. A computational example using a nonlinear quantity of interest (solution energy) is also provided in Section 4. The theory will be restricted to the case that $\psi$ is smooth. This requires some lieniency in defining a quantity of interest. An example is the average over a subset $S \subset \Omega_{T}$, for which the data $\psi$ would be

$$
\psi(x, t)=\frac{1}{|S|} \chi_{S}(x, t)
$$

The function $\chi_{S}$ is the characteristic function for the set $S$. Instead, one could simply replace the characteristic function with something that smoothly transitions between 0 and 1 near the boundary of $S$ and choose to study the error in that quantity of interest. Similarly, instead of solution value at a point, one could study a weighted average over a small region centered at the point of interest. In Section 4 computational examples are provided in the case that $\psi$ is not smooth, for reference.

It is assumed that a finite volume method has been applied to estimate the solution of (1.1)-(1.2), yielding a data set denoted by $\bar{v}$. The quantity of interest is then estimated in two steps:

(1) Define a pointwise reconstruction $\tilde{u}$ from the finite volume data set $\bar{v}$.

(2) Calculate $\mathcal{M}(\tilde{u})$; this is the approximation of $\mathcal{M}(u)$.

In practice, no method exists to calculate the error $\mathcal{M}(u)-\mathcal{M}(\tilde{u})$ exactly, since the true solution is not known. There are a number of techniques for estimating the error, (see e.g. $[19,20]$ ), one of which is adjoint error estimation. This technique requires deriving an auxiliary (adjoint) problem for the error in the quantity of interest. If the solution of the adjoint problem were known exactly, the error in the quantity of interest could be estimated to almost machine precision. The theory has been well developed using a variety of numerical methods for elliptic and parabolic problems, e.g. $[10,18]$. Numerous numerical studies exist for application to hyperbolic conservation laws, e.g. [3-7, 11, 13, 15-17, 22]. However, there are still few rigorous theoretical results in the context of hyperbolic conservation laws (mainly [18]), and none known to the authors that address shock problems estimated using finite volume methods. A thorough description of the current state 
of adjoint based error estimation techniques can be found in the review papers of Giles and Süli [14] as well as Fidkowski and Darmofal [12].

The desired application is to quantify uncertainty in computational models requiring complex black box codes to be run. Thus an emphasis is placed on developing a nonintrusive framework for a posteriori error estimation. This report introduces such a technique in Section 2, that may lead to adjoint error estimation codes that can be implemented as separate modules attached to a black box code. The only requirement is that the forward problem has a governing set of equations, initial condition and boundary conditions associated with it known to the developers of the adjoint code. The application to a simple linear advection problem is described in Section 3. A rigorous proof of convergence is provided for the computable error estimate in the case of smooth solutions of the forward and adjoint problems. In Section 4 the theory is explored in numerous computational examples, including cases involving discontinuities. Conclusions and suggested future work are provided in Section 5.

\section{The ADJOINT PROBlem AND ERROR FORMUlA}

This section introduces a modification of the approach previously taken in numerous studies, most notably [4-6], to develop an adjoint problem for the error in a quantity of interest when estimating the solution of a hyperbolic conservation law using a finite volume method. In these studies the forward problem is derived by proving an equivalency of the finite volume method with some finite element method, then enriching the trial and test spaces to extract a definition of a weak operator. A different approach is possible assuming solutions with regularity $u \in H^{1}\left(\Omega_{T}\right)$ and forcing $F \in L^{2}\left(\Omega_{T}\right)$. Consider developing a weak problem over trial and test spaces containing a large class of approximating function spaces and the solution itself. Reconstructions from finite volume data may be included by allowing jumps across cell interfaces in the trial space. Denote a set of cells with maximum cell width $h$ by $\mathcal{K}^{h}$, where the cells are denoted by

$$
\mathcal{K}_{i}^{h}=\left(x_{i-1 / 2}, x_{i+1 / 2}\right), i=1, \ldots, M,
$$

such that $x_{1 / 2}=x_{L}$ and $x_{M+1 / 2}=x_{R}$. Cell centers are denoted by $x_{i}, i=$ $1, \ldots, M$. The exact cell averages of the solution $u$ of (1.1)-(1.2) are denoted by $\left\{\bar{u}_{i}^{n}\right\}$, satisfying

$$
\bar{u}_{i}^{n}=\frac{1}{x_{i+1 / 2}-x_{i-1 / 2}} \int_{x_{i-1 / 2}}^{x_{i+1 / 2}} u\left(x, t^{n}\right) d x
$$

on each cell $\mathcal{K}_{i}^{h}$ at each discrete time $t^{n} \in\left\{0=t^{0}, t^{1}, \ldots, t^{N}=T\right\}$. These cell averages are approximated by a finite volume data set $\bar{v}=\left\{\bar{v}_{i}^{n}\right\}$. The desired function space is taken to be the broken space [6]

$$
\mathcal{V}^{B}=\left\{\left.v\right|_{\mathcal{K}_{i}^{h}} \in H^{1}\left(\mathcal{K}_{i}^{h} \times(0, T)\right), i=1, \ldots, M\right\} .
$$

The norm on $\mathcal{V}^{B}$ is

$$
\|v\|_{\mathcal{V}^{B}}=\left\{\sum_{i=1}^{M} \int_{\mathcal{K}_{i}^{h} \times(0, T)}|v(x, t)|^{2}+\left|\partial_{t} v(x, t)\right|^{2}+\left|\partial_{x} v(x, t)\right|^{2} d x d t\right\}^{1 / 2}, \forall v \in \mathcal{V}^{B} .
$$


The norm for $L^{2}\left(\Omega_{T}\right)$ is denoted in the usual way by $\|\cdot\|_{L^{2}\left(\Omega_{T}\right)}$. A trivial extension operator $T: v \in \mathcal{V}^{B} \rightarrow T(v) \in L^{2}\left(\Omega_{T}\right)$ exists, so for convenience the notation is suppressed by saying $\mathcal{V}^{B}=T\left(\mathcal{V}^{B}\right) \subset L^{2}\left(\Omega_{T}\right)$. At each cell interface define left and right values by

$$
\begin{aligned}
& v_{-}(x)=\lim _{y \rightarrow x^{-}} v(y), \\
& v_{+}(x)=\lim _{y \rightarrow x^{+}} v(y) .
\end{aligned}
$$

2.1. Identification of an adjoint problem for the error. First multiply (1.1) by $v \in \mathcal{V}^{B}$ and integrate by parts over $\mathcal{K}_{i}^{h} \times(0, T)$ :

$$
\begin{aligned}
\int_{\mathcal{K}_{i}^{h} \times(0, T)} \partial_{t} u v d x d t- & \int_{\mathcal{K}_{i}^{h} \times(0, T)} f(u) \partial_{x} v d x d t+\int_{0}^{T} f\left(u\left(x_{i+1 / 2}\right)\right) v_{-}\left(x_{i+1 / 2}\right) d t \\
& -\int_{0}^{T} f\left(u\left(x_{i-1 / 2}\right)\right) v_{+}\left(x_{i-1 / 2}\right) d t=\int_{\mathcal{K}_{i}^{h} \times(0, T)} F v d x d t .
\end{aligned}
$$

The development of the adjoint problem will require a precise description of what happens when we add a perturbation $w \in \mathcal{V}^{B}$ to $u$, and in this case the flux across the cell interfaces must be well defined. Hence we replace the flux in (2.5) with some rule based on the left and right values at the cell interface, denoted by $\hat{f}\left(w_{-}\left(x_{i-1 / 2}\right), w_{+}\left(x_{i-1 / 2}\right)\right)$, for $i=0,1, \ldots, M$. The rule must be consistent, so that for the true solution $u \in H^{1}\left(\Omega_{T}\right)$ :

$$
\hat{f}\left(u_{-}\left(x_{i-1 / 2}\right), u_{+}\left(x_{i-1 / 2}\right)=f\left(u\left(x_{i-1 / 2}\right)\right), i=1, \ldots, M+1 .\right.
$$

Inserting this flux rule into (2.5) and summing over $i=1, \ldots, M$,

$$
A(u, v)=\sum_{i=1}^{M} \int_{\mathcal{K}_{i}^{h} \times(0, T)} F v d x d t, \forall v \in \mathcal{V}^{B},
$$

where

$$
\begin{aligned}
A(u, v)= & \sum_{i=1}^{M} \int_{\mathcal{K}_{i}^{h} \times(0, T)} \partial_{t} u v d x d t-\sum_{i=1}^{M} \int_{\mathcal{K}_{i}^{h} \times(0, T)} f(u) \partial_{x} v d x d t \\
& +\sum_{i=1}^{M} \int_{0}^{T} \hat{f}\left(u_{-}\left(x_{i+1 / 2}\right), u_{+}\left(x_{i+1 / 2}\right)\right) v_{-}\left(x_{i+1 / 2}\right) d t \\
& -\sum_{i=1}^{M} \int_{0}^{T} \hat{f}\left(u_{-}\left(x_{i-1 / 2}\right), u_{+}\left(x_{i-1 / 2}\right)\right) v_{+}\left(x_{i-1 / 2}\right) d t .
\end{aligned}
$$

This method of extracting a weak operator consistent with the strong formulation for smooth solutions is equivalent to developing a local DG method as described by Cockburn et. al., $[8,9]$, and enriching the trial and test spaces. The adjoint problem is now derived by linearization about an approximation $\tilde{u} \in \mathcal{V}^{B}$ of the solution to 
(1.1). Indeed,

$$
\begin{gathered}
A(u, v)-A(\tilde{u}, v)=\int_{0}^{1} \frac{d}{d s} A(\tilde{u}+s(u-\tilde{u}), v) d s \\
=\sum_{i=1}^{M} \int_{\mathcal{K}_{i}^{h} \times(0, T)} \partial_{t}(u-\tilde{u}) v d x d t-\sum_{i=1}^{M} \int_{\mathcal{K}_{i}^{h} \times(0, T)} g(u, \tilde{u})(u-\tilde{u}) \partial_{x} v d x d t \\
+\left.\sum_{i=1}^{M} \int_{0}^{T}\{\hat{g}(u, \tilde{u})(u-\tilde{u})\}\right|_{x_{i+1 / 2}} v_{-}\left(x_{i+1 / 2}\right) d t \\
-\left.\sum_{i=1}^{M} \int_{0}^{T}\{\hat{g}(u, \tilde{u})(u-\tilde{u})\}\right|_{x_{i-1 / 2}} v_{+}\left(x_{i-1 / 2}\right) d t,
\end{gathered}
$$

where it is assumed the Fréchet derivatives $g, \hat{g}$ exist, satisfying:

$$
g(u, \tilde{u})(u-\tilde{u})=\left(\int_{0}^{1} \partial_{u} f(\tilde{u}+s(u-\tilde{u})) d s\right)(u-\tilde{u})
$$

and

$$
\begin{aligned}
& \left.\{\hat{g}(u, \tilde{u})(u-\tilde{u})\}\right|_{x_{i+1 / 2}} \\
& =\left.\int_{0}^{1}\left\{\partial_{u} \hat{f}\left((\tilde{u}+s(u-\tilde{u}))_{-},(\tilde{u}+s(u-\tilde{u}))_{+}\right)(u-\tilde{u})\right\}\right|_{x_{i+1 / 2}} d s .
\end{aligned}
$$

Consider $w \in \mathcal{V}^{B}$ to be an arbitrary perturbation inserted in place of $u-\tilde{u}$ in (2.9). The linearized variational form is

$$
\begin{aligned}
\bar{A}(u, \tilde{u}, w, v) & =\sum_{i=1}^{M} \int_{\mathcal{K}_{i}^{h} \times(0, T)} \partial_{t} w v d x d t-\sum_{i=1}^{M} \int_{\mathcal{K}_{i}^{h} \times(0, T)} g(u, \tilde{u}) w \partial_{x} v d x d t \\
& +\left.\sum_{i=1}^{M} \int_{0}^{T}\{\hat{g}(u, \tilde{u}) w\}\right|_{x_{i+1 / 2}} v_{-}\left(x_{i+1 / 2}\right) d t \\
& -\left.\sum_{i=1}^{M} \int_{0}^{T}\{\hat{g}(u, \tilde{u}) w\}\right|_{x_{i-1 / 2}} v_{+}\left(x_{i-1 / 2}\right) d t .
\end{aligned}
$$

Integrating (2.10) by parts one attempts to determine appropriate boundary conditions and a terminal time condition so that the adjoint problem is well-posed. We cannot describe this for the general case above, though Section 3 will provide a specific example. We leave the investigation of when this can be done as future work. In the case of periodic boundary conditions an adjoint variational form is derived, denoted here by

$$
\bar{A}^{*}(u, \tilde{u}, v, w)=\bar{A}(u, \tilde{u}, w, v)+\int_{\Omega} w(x, 0) v(x, 0) d x-\int_{\Omega} w(x, T) v(x, T) d x .
$$

Two cases are considered here corresponding to the two forms of the quantity of interest described in (1.3)-(1.4). In the case $\mathcal{M}(u)$ is of the form (1.3), the adjoint problem is to solve for $\phi \in \mathcal{V}^{B}$ satisfying

$$
\bar{A}^{*}(u, \tilde{u}, \phi, w)=\mathcal{M}(w), \forall w \in \mathcal{V}^{B}
$$

with periodic boundary conditions, subject to the terminal condition $\phi(x, T)=0$. If $\mathcal{M}(u)$ is of the form (1.4), the adjoint problem is to solve for $\phi \in \mathcal{V}^{B}$ satisfying

$$
\bar{A}^{*}(u, \tilde{u}, \phi, w)=0, \forall w \in \mathcal{V}^{B}
$$


with periodic boundary conditions, subject to the terminal condition $\phi(x, T)=\psi(x)$ in $\Omega$.

2.2. The error representation formula. The goal is to derive an error formula that can be decomposed into computable and noncomputable terms, where the noncomputable terms should be asymptotically small compared to the computable terms. This is accomplished by introducing some element $\tilde{\phi} \in \mathcal{V}^{B}$ that is considered to be an approximation of the adjoint solution $\phi$. If $\mathcal{M}(u)$ is of the form $(1.3)$, the error in the quantity of interest is

$$
\begin{gathered}
\mathcal{M}(u)-\mathcal{M}(\tilde{u})=\mathcal{M}(u-\tilde{u})=\bar{A}^{*}(u, \tilde{u}, \phi, u-\tilde{u}) \\
=\bar{A}(u, \tilde{u}, u-\tilde{u}, \phi)+\int_{\Omega}(u-\tilde{u})(x, 0) \phi(x, 0) d x \\
=\bar{A}(u, \tilde{u}, u-\tilde{u}, \phi-\tilde{\phi})+\int_{\Omega}(u-\tilde{u})(x, 0)(\phi-\tilde{\phi})(x, 0) d x \\
\quad+\int_{\Omega}(u-\tilde{u})(x, 0) \tilde{\phi}(x, 0) d x+\bar{A}(u, \tilde{u}, u-\tilde{u}, \tilde{\phi}) \\
=\bar{A}(u, \tilde{u}, u-\tilde{u}, \phi-\tilde{\phi})+\int_{\Omega}(u-\tilde{u})(x, 0)(\phi-\tilde{\phi})(x, 0) d x \\
\quad+\int_{\Omega}(u-\tilde{u})(x, 0) \tilde{\phi}(x, 0) d x+A(u, \tilde{\phi})-A(\tilde{u}, \tilde{\phi}) \\
=\bar{A}(u, \tilde{u}, u-\tilde{u}, \phi-\tilde{\phi})+\int_{\Omega}\left(u_{0}(x)-\tilde{u}(x, 0)\right)(\phi-\tilde{\phi})(x, 0) d x \\
+\int_{\Omega}\left(u_{0}(x)-\tilde{u}(x, 0)\right) \tilde{\phi}(x, 0) d x+\int_{\Omega_{T}} F(x, t) \tilde{\phi}(x, t) d x d t-A(\tilde{u}, \tilde{\phi}) .
\end{gathered}
$$

If $\mathcal{M}(u)$ is of the form (1.4), the same result is achieved using a slight modification of these arguments. In practice one estimates the error using only the computable terms:

$\mathcal{M}(u)-\mathcal{M}(\tilde{u}) \approx \int_{\Omega}\left(u_{0}(x)-\tilde{u}(x, 0)\right) \tilde{\phi}(x, 0) d x+\int_{\Omega_{T}} F(x, t) \tilde{\phi}(x, t) d x d t-A(\tilde{u}, \tilde{\phi})$.

The merit of the error approximation (2.15) lies in the asymptotic properties of the noncomputable terms. As an example, if the quantity of interest is determined by (1.3), the size of the true error is expected to be

$$
\mathcal{M}(u)-\mathcal{M}(\tilde{u}) \approx C_{1}\left(\Omega_{T}\right)\|u-\tilde{u}\|_{L^{p}\left(\Omega_{T}\right)}
$$

for some $p$ such that $1 \leq p \leq 2$. If a uniform bound on the size of the derivatives $g$ and $\hat{g}$ can be shown, the size of the noncomputable terms is expected to be

$$
\bar{A}(u, \tilde{u}, u-\tilde{u}, \phi-\tilde{\phi}) \approx C_{2}\left(\Omega_{T}\right)\|u-\tilde{u}\|_{\mathcal{V}^{B}}\|\phi-\tilde{\phi}\|_{\mathcal{V}^{B}} .
$$

Comparing (2.16) and (2.17), it is evident that the computable error estimate can achieve any desired accuracy by choosing $\tilde{\phi}$ to be a sufficiently close approximation of $\phi$ in the norm of $\mathcal{V}^{B}$.

Remark 2.1. The a posteriori error formula is usually used to derive local information regarding the size of the error to use in mesh refinement. In the current setting this is not possible as the required Galerkin orthogonality is lost. The advantage of the above construction is to separate the method of estimating the solution $u$ from 
the method of estimating the adjoint solution $\phi$. The advantage of this approach in terms of both computational efficiency and programmer effort is illustrated in the following sections.

\section{Application to COnStant-Coefficient linear adVEction}

An adjoint error estimate of the form (2.15) is derived in the case $u$ solves

$$
\begin{aligned}
\partial_{t} u+a \partial_{x} u & =0,(x, t) \in \Omega_{T} \\
u(x, 0) & =u_{0}(x), x \in \Omega,
\end{aligned}
$$

with periodic boundary conditions. The choice of periodic boundary conditions is just a first step, and we do not consider the issue of boundary conditions to be essential in 1D. The advection coefficient is assumed to be positive. The equation is unforced for simplicity, which does not effect the analysis. However, it makes the development of code for computational tests easier in Section 4. The condition $u_{0}(x) \in H^{1}(\Omega)$ is imposed, in which case the solution $u(x, t)=u_{0}(x-a t) \in$ $H^{1}\left(\Omega_{T}\right) \subset \mathcal{V}^{B}$. The flux function is simply $f(u)=a u$. The development of the adjoint problem is greatly simplified in this case by choosing the flux rule at cell interfaces for arbitrary $w \in \mathcal{V}^{B}$ to be the upwind flux (for $a>0$ ):

$$
\hat{f}\left(w_{-}\left(x_{i-1 / 2}\right), w_{+}\left(x_{i-1 / 2}\right)=a w_{-}\left(x_{i-1 / 2}\right), i=1, \ldots, M+1 .\right.
$$

The variational form (2.8) becomes

$$
\begin{aligned}
& A(u, v)=\sum_{i=1}^{M} \int_{\mathcal{K}_{i}^{h} \times(0, T)} \partial_{t} u v d x d t-\sum_{i=1}^{M} \int_{\mathcal{K}_{i}^{h} \times(0, T)} a u \partial_{x} v d x d t \\
&+\sum_{i=1}^{M} \int_{0}^{T} a u_{-}\left(x_{i+1 / 2}\right) \\
& v_{-}\left(x_{i+1 / 2}\right) d t \\
&-\sum_{i=1}^{M} \int_{0}^{T} a u_{-}\left(x_{i-1 / 2}\right) v_{+}\left(x_{i-1 / 2}\right) d t
\end{aligned}
$$

and if $u \in H^{1}\left(\Omega_{T}\right)$ solves (3.1), the variational form satisfies

$$
A(u, v)=0, \forall v \in \mathcal{V}^{B} .
$$

There is no need to perform any linearization. If $\tilde{u} \in \mathcal{V}^{B}$ is chosen arbitrarily and

$w=u-\tilde{u}$,

$$
\begin{gathered}
A(u, v)-A(\tilde{u}, v)=A(w, v)=\sum_{i=1}^{M} \int_{\mathcal{K}_{i}^{h} \times(0, T)} \partial_{t} w v d x d t-\sum_{i=1}^{M} \int_{\mathcal{K}_{i}^{h} \times(0, T)} a w \partial_{x} v d x d t \\
+\sum_{i=1}^{M} \int_{0}^{T} a w_{-}\left(x_{i+1 / 2}\right) v_{-}\left(x_{i+1 / 2}\right) d t-\sum_{i=1}^{M} \int_{0}^{T} a w_{-}\left(x_{i-1 / 2}\right) v_{+}\left(x_{i-1 / 2}\right) d t .
\end{gathered}
$$


Integrating by parts,

$$
\begin{gathered}
A(w, v)=\sum_{i=1}^{M} \int_{\mathcal{K}_{i}^{h} \times(0, T)}-\partial_{t} v w d x d t+\sum_{i=1}^{M} \int_{\mathcal{K}_{i}^{h} \times(0, T)} a v \partial_{x} w d x d t \\
\quad+\int_{\Omega} w(x, T) v(x, T) d x-\int_{\Omega} w(x, 0) v(x, 0) d x \\
+\sum_{i=1}^{M} \int_{0}^{T} a w_{+}\left(x_{i-1 / 2}\right) v_{+}\left(x_{i-1 / 2}\right) d t-\sum_{i=1}^{M} \int_{0}^{T} a w_{-}\left(x_{i-1 / 2}\right) v_{+}\left(x_{i-1 / 2}\right) d t
\end{gathered}
$$

The adjoint variational form is obtained in the standard way by dropping the terms integrated in space at the initial and final times. The boundary conditions for the adjoint variable are taken to be periodic. The reason for this will be made clear after first rewriting the sum over the cell interface terms and applying the periodic boundary conditions,

$$
\begin{aligned}
& \sum_{i=1}^{M} \int_{0}^{T} a w_{+}\left(x_{i-1 / 2}\right) v_{+}\left(x_{i-1 / 2}\right) d t \\
- & \sum_{i=1}^{M} \int_{0}^{T} a w_{-}\left(x_{i-1 / 2}\right) v_{+}\left(x_{i-1 / 2}\right) d t=\sum_{i=1}^{M} \int_{0}^{T} a w_{+}\left(x_{i-1 / 2}\right) v_{+}\left(x_{i-1 / 2}\right) d t \\
& -\sum_{i=1}^{M-1} \int_{0}^{T} a w_{-}\left(x_{i+1 / 2}\right) v_{+}\left(x_{i+1 / 2}\right) d t-\int_{0}^{T} a w_{-}\left(x_{1 / 2}\right) v_{+}\left(x_{1 / 2}\right) d t \\
= & \sum_{i=1}^{M} \int_{0}^{T} a w_{+}\left(x_{i-1 / 2}\right) v_{+}\left(x_{i-1 / 2}\right) d t-\sum_{i=1}^{M} \int_{0}^{T} a w_{-}\left(x_{i+1 / 2}\right) v_{+}\left(x_{i+1 / 2}\right) d t .
\end{aligned}
$$

The adjoint variational form is now

$$
\begin{gathered}
A^{*}(v, w)=\sum_{i=1}^{M} \int_{\mathcal{K}_{i}^{h} \times(0, T)}-\partial_{t} v w d x d t+\sum_{i=1}^{M} \int_{\mathcal{K}_{i}^{h} \times(0, T)} a v \partial_{x} w d x d t \\
+\sum_{i=1}^{M} \int_{0}^{T} a w_{+}\left(x_{i-1 / 2}\right) v_{+}\left(x_{i-1 / 2}\right) d t \\
\quad-\sum_{i=1}^{M} \int_{0}^{T} a w_{-}\left(x_{i+1 / 2}\right) v_{+}\left(x_{i+1 / 2}\right) d t .
\end{gathered}
$$

Throughout the remainder of this report, only quantities of interest of the form (1.4) are considered. The adjoint problem is to find $\phi \in \mathcal{V}^{B}$ satisfying

$$
A^{*}(\phi, w)=0, \forall w \in \mathcal{V}^{B},
$$

subject to periodic boundary conditions and the terminal condition $\phi(x, T)=\psi(x)$. The importance of this problem formulation lies in the observation that if $\psi(x) \in$ $H^{1}(\Omega)$, then $\phi(x, t) \in H^{1}\left(\Omega_{T}\right)$ satisfying the linear advection problem

$$
\begin{aligned}
-\partial_{t} \phi+\partial_{x} f_{a d j}(\phi) & =0,(x, t) \in \Omega_{T} \\
f_{a d j}(\phi) & =-a \phi, \\
\phi(x, T) & =\psi(x), x \in \Omega
\end{aligned}
$$


will also satisfy (3.10). This is proved by simply multiplying (3.11) by any $w \in \mathcal{V}^{B}$ and integrating the advection term by parts and applying the upwind flux rule

$$
\left.\hat{f}_{a d j}(-a v)\right|_{x_{i+1 / 2}}(t)=-a v_{+}\left(x_{i+1 / 2}, t\right) .
$$

Thus for a large class of quantities of interest the solvability of the adjoint problem is trivial. Also, less smooth choices of $\psi(x)$ that may be of interest, like $\delta$-functions or step functions, can easily be approximated by $\psi \approx \psi_{\epsilon} \in H^{1}(\Omega)$ or even smooth functions. Denote by $e_{\mathcal{M}}(\tilde{u})$ the error in the quantity of interest,

$$
e_{\mathcal{M}}(\tilde{u})=\mathcal{M}(u)-\mathcal{M}(\tilde{u}) .
$$

The error formula (2.14) simplifies to

$$
\begin{aligned}
e_{\mathcal{M}}(\tilde{u})=\mathcal{M}(u-\tilde{u})=A(u-\tilde{u}, \phi-\tilde{\phi}) & +\int_{\Omega}\left(u_{0}(x)-\tilde{u}(x, 0)\right)(\phi-\tilde{\phi})(x, 0) d x \\
& +\int_{\Omega}\left(u_{0}(x)-\tilde{u}(x, 0)\right) \tilde{\phi}(x, 0) d x-A(\tilde{u}, \tilde{\phi}) .
\end{aligned}
$$

The computable error estimate is denoted by $\tilde{e}_{\mathcal{M}}(\tilde{u}, \tilde{\phi})$ and is calculated using the rule

$$
e_{\mathcal{M}}(\tilde{u}) \approx \tilde{e}_{\mathcal{M}}(\tilde{u}, \tilde{\phi})=\int_{\Omega}\left(u_{0}(x)-\tilde{u}(x, 0)\right) \tilde{\phi}(x, 0) d x-A(\tilde{u}, \tilde{\phi}) .
$$

3.1. Reconstruction mappings for finite volume data. The approximation $\tilde{u}$ of $u$ could be constructed in many ways. A map from the finite volume data set $\bar{v}$ to a space-time approximation $\tilde{u} \in \mathcal{V}^{B}$ is constructed here to achieve an optimal order of approximation for the desired quantity of interest. First, pointwise values in space are defined at each discrete time on each cell, then a piecewise interpolant of the data in time is used to define $\tilde{u}$.

A common method of data reconstruction in space is used (see e.g. Shu, [21]), which yields piecewise polynomials, continuous on each cell with jumps across cell interfaces. The spatial reconstruction of the finite volume data $\bar{u}$ at time $t^{n}$ is denoted by $\tilde{u}^{n}(x)$, where $\left.\tilde{u}^{n}(x)\right|_{\mathcal{K}_{i}^{h}}=\tilde{u}_{i}^{n}(x)$ and the polynomial $\tilde{u}_{i}^{n}(x)$ satisfies

$$
\int_{\mathcal{K}_{j}^{h}} \tilde{u}_{i}^{n}(x) d x=\left(x_{j+1 / 2}-x_{j-1 / 2}\right) \bar{v}_{j}^{n}, j=i-r, i-r+1, \ldots, i-r+p .
$$

The shifting index $r, 0 \leq r \leq p$, is fixed independent of $i$ for convenience since the solution is periodic. In the presence of boundary conditions the stencil can be shifted toward the interior of the domain without affecting the asymptotic properties proved in this report. Given the reconstructions $\tilde{u}^{n}(x)$ for time indices $n=0,1, \ldots, N$, the final space-time reconstructed solution $\tilde{u}(x, t)$ is constructed by interpolation in time. If $x \in \mathcal{K}_{i}^{h}$ and $0 \leq n-r \leq N-p-1, \tilde{u}(x, t)$ is defined on $\left[t^{n}, t^{n+1}\right]$ as the unique polynomial interpolant of degree $p+1$ satisfying

$$
\tilde{u}\left(x, t^{j}\right)=\tilde{u}_{i}^{j}(x), j=n-r, n-r+1, \ldots, n-r+p+1 .
$$

In case the interval $\left[t^{n}, t^{n+1}\right]$ is near the initial or final times the value of $r$ is adjusted accordingly. The reason the interpolant in time is of degree $p+1$ will be clarified in Lemma 3.1.

Usually reconstruction operators are used to design finite volume methods of a desired accuracy. However, the proposed reconstruction process can be used 
to obtain information about a quantity of interest and the associated error to an optimal order of accuracy without knowing the original reconstruction process used in designing the finite volume method, assuming a smooth solution to the forward and adjoint problems. The polynomial degree $p$ can be chosen based on the accuracy of the finite volume method to ensure an optimal rate of convergence for smooth solutions. Since the accuracy of the computable error estimate satisfies (3.48), this means the first derivative in space and in time of the reconstruction should ideally converge at the same rate as the finite volume data set. This motivates the following result. In order to ease the proof, define a maximum cell width $\Delta x$ and time step size $\Delta t$

$$
\begin{aligned}
\Delta x & =\max _{i=1,2, \ldots, M}\left(\Delta x_{i}=x_{i+1 / 2}-x_{i-1 / 2}\right) \\
\Delta t & =\max _{n=0,1, \ldots, N-1}\left(\Delta t^{n+1}=t^{n+1}-t^{n}\right),
\end{aligned}
$$

and a CFL condition

$$
\frac{a \Delta t^{n+1}}{\Delta x_{i}} \leq \lambda
$$

for $\lambda \in(0,1)$, independent of $n$ and $i$.

Lemma 3.1. Let $\bar{w}_{j}^{n}$ be a finite volume data set approximating some smooth function $U(x, t)$ with periodic boundary conditions in space and denote the cell average errors by

$$
\bar{e}_{j}^{n}=\bar{U}_{j}^{n}-\bar{w}_{j}^{n}
$$

where $\bar{U}_{j}^{n}$ satisfies

$$
\bar{U}_{j}^{n}=\frac{1}{x_{j+1 / 2}-x_{j-1 / 2}} \int_{x_{j-1 / 2}}^{x_{j+1 / 2}} U\left(x, t^{n}\right) d x .
$$

Assume a regular series of nodes $\left\{x_{i-1 / 2}\right\}$ such that there exists $\alpha>0$ satisfying

$$
\begin{aligned}
\alpha \Delta x & \leq \min _{i=1,2, \ldots, M} \Delta x_{i} \\
\alpha \Delta t & \leq \min _{n=0,1, \ldots, N-1} \Delta t^{n+1}
\end{aligned}
$$

independent of $N$ or $M$, and that the following approximation properties hold for constants $C_{0}, C_{1}>0$ and $C_{2}>0$ independent of $N$ or $M$ :

$$
\begin{aligned}
\max _{n=1,2, \ldots, N} \max _{j=1,2, \ldots, M}\left|\bar{e}_{j}^{n}\right| & \leq C_{0} \Delta x^{s}, \\
\max _{n=1,2, \ldots, N} \max _{j=2,3, \ldots, M}\left|\bar{e}_{j}^{n}-\bar{e}_{j-1}^{n}\right| & \leq C_{1} \Delta x^{s+1}, \\
\text { and } \max _{n=0,2, \ldots, N-1} \max _{j=2,3, \ldots, M}\left|\bar{e}_{j}^{n+1}-\bar{e}_{j}^{n}\right| & \leq C_{2} \Delta x^{s+1} .
\end{aligned}
$$

Let $\tilde{U}$ be the space-time reconstruction of $U$ as defined in (3.16)-(3.17) using piecewise polynomials of degree $p$ in space and a stencil shift index $r$. Then the following 
approximation properties hold:

$$
\begin{aligned}
\|U-\tilde{U}\|_{L^{\infty}\left(\Omega_{T}\right)} & =\mathcal{O}\left(\Delta x^{p+1}+\Delta x^{s}\right), \\
\left\|\partial_{x}(U-\tilde{U})\right\|_{L^{\infty}\left(\Omega_{T}\right)} & =\mathcal{O}\left(\Delta x^{p}+\Delta x^{s}\right), \\
\left\|\partial_{t}(U-\tilde{U})\right\|_{L^{\infty}\left(\Omega_{T}\right)} & =\mathcal{O}\left(\Delta x^{p+1}+\Delta x^{s}\right), \\
\text { and } \max _{i=1, \ldots, M}\left|[U-\tilde{U}]\left(x_{i+1 / 2}\right)\right| & =\mathcal{O}\left(\Delta x^{p+1}+\Delta x^{s+1}\right) .
\end{aligned}
$$

Remark 3.1. The conditions (3.20)-(3.21) could be removed if the approximation properties (3.22)-(3.23) were localized. Also, condition (3.24) should follow automatically from (3.23) when employing finite volume methods with hyperbolic conservation laws due to the CFL condition. The conditions can be easily proved for many finite volume methods by writing the cell average errors in terms of the local truncation errors.

Proof. Uniform bounds will be derived on an arbitrary cell $\mathcal{K}_{i}^{h}$ and time $t^{n}$. Let $q_{i}^{n}(x)$ be the polynomial interpolant of degree $p+1$ satisfying

$$
\begin{aligned}
q_{i}^{n}\left(x_{i-r+j-1 / 2}\right)=\int_{x_{1 / 2}}^{x_{i-r+j-1 / 2}} U(\tau, & \left.t^{n}\right) d \tau \\
& =\sum_{k=1}^{i-r+j-1} \Delta x_{k} \bar{U}_{k}^{n}, j=0,1, \ldots, p+1 .
\end{aligned}
$$

Consider the approximate interpolant based on the finite volume data set

$$
\tilde{q}_{i}^{n}\left(x_{i-r+j-1 / 2}\right)=\sum_{k=1}^{i-r+j-1} \Delta x_{k} \bar{w}_{k}^{n}, j=0,1, \ldots, p+1 .
$$

Since $U$ is smooth, it is well known that $\partial_{x}^{(d)} U\left(x, t^{n}\right)-\partial_{x}^{(d+1)} q_{i}^{n}(x)=\mathcal{O}\left(\Delta x^{p+1-d}\right)$ for $d=0,1$. It will be shown that

$$
\max _{i=1,2, \ldots, M} \max _{x \in \mathcal{K}_{i}^{h}}\left(\partial_{x}^{(d)} q_{i}^{n}(x)-\partial_{x}^{(d)} \tilde{q}_{i}^{n}(x)\right)=\mathcal{O}\left(\Delta x^{s}\right)
$$

for $d=1,2$. Let the polynomial $e_{i}^{n}(x)$ interpolate the errors at each cell interface,

$$
\begin{aligned}
e_{i}^{n}\left(x_{i-r+j-1 / 2}\right)=q_{i}^{n}\left(x_{i-r+j-1 / 2}\right)- & \tilde{q}_{i}^{n}\left(x_{i-r+j-1 / 2}\right) \\
& =\sum_{k=1}^{i-r+j-1} \Delta x_{k} \bar{e}_{k}^{n}, j=0,1, \ldots, p+1 .
\end{aligned}
$$

The Newton divided difference form of the interpolant (Atkinson [2]) $e_{i}^{n}(x)$ is

$$
\begin{aligned}
& e_{i}^{n}(x)=e_{i}^{n}\left(x_{i-r-1 / 2}\right)+\left(x-x_{i-r-1 / 2}\right) e_{i}^{n}\left[x_{i-r-1 / 2}, x_{i-r+1 / 2}\right] \\
& \quad+\left(x-x_{i-r-1 / 2}\right)\left(x-x_{i-r+1 / 2}\right) e_{i}^{n}\left[x_{i-r-1 / 2}, x_{i-r+1 / 2}, x_{i-r+3 / 2}\right] \\
& \quad+\ldots+\prod_{m=0}^{p}\left(x-x_{i-r+m-1 / 2}\right) e_{i}^{n}\left[x_{i-r-1 / 2}, x_{i-r+1 / 2}, \ldots, x_{i-r+p+1 / 2}\right] .
\end{aligned}
$$

The divided difference notation $e_{i}^{n}\left[x_{i-r+l-1 / 2}, x_{i-r+l+1 / 2}, \ldots, x_{i-r+l+j+1 / 2}\right]$ for $j=$ $0,1, \ldots, p$ and $l=0, \ldots, p-j$ is defined first for case $j=0$ by setting

$$
e_{i}^{n}\left[x_{i-r+l-1 / 2}, x_{i-r+l+1 / 2}\right]=\frac{e_{i}^{n}\left(x_{i-r+l+1 / 2}\right)-e_{i}^{n}\left(x_{i-r+l-1 / 2}\right)}{x_{i-r+l+1 / 2}-x_{i-r+l-1 / 2}} \text { for } l=0,1, \ldots, p .
$$


Then inductively with respect to $j$,

$$
\begin{aligned}
& e_{i}^{n}\left[x_{i-r+l-1 / 2}, \ldots, x_{i-r+l+j+1 / 2}\right] \\
= & \frac{e_{i}^{n}\left[x_{i-r+l+1 / 2}, \ldots, x_{i-r+l+j+1 / 2}\right]-e_{i}^{n}\left[x_{i-r+l-1 / 2}, \ldots, x_{i-r+l+j-1 / 2}\right]}{x_{i-r+l+j+1 / 2}-x_{i-r+l-1 / 2}} \quad \text { for } l=0,1, \ldots, p-j .
\end{aligned}
$$

These expressions can be calculated in terms of the cell average errors using (3.32). In case $j=0$ one obtains

$$
e_{i}^{n}\left[x_{i-r+l-1 / 2}, x_{i-r+l+1 / 2}\right]=\bar{e}_{i-r+l}^{n} \text { for } l=0,1, \ldots, p .
$$

Inserting the result (3.35) into (3.34) in case $j=1$ yields

$$
\begin{aligned}
& e_{i}^{n}\left[x_{i-r+l-1 / 2}, x_{i-r+l+1 / 2}, x_{i-r+l+3 / 2}\right] \\
& =\frac{\bar{e}_{i-r+l+1}^{n}-\bar{e}_{i-r+l}^{n}}{x_{i-r+l+3 / 2}-x_{i-r+l-1 / 2}} \text { for } l=0,1, \ldots, p-1 .
\end{aligned}
$$

An upper bound is obtained using (3.20) and (3.23):

$$
\begin{aligned}
\left|e_{i}^{n}\left[x_{i-r+l-1 / 2}, x_{i-r+l+1 / 2}, x_{i-r+l+3 / 2}\right]\right| & \\
\leq & \frac{C_{1} \Delta x^{s+1}}{2 \alpha \Delta x} \text { for } l=0,1, \ldots, p-1 .
\end{aligned}
$$

Induction on $j$ is used to prove the upper bound

$$
\left|e_{i}^{n}\left[x_{i-r+l-1 / 2}, \ldots, x_{i-r+l+j+1 / 2}\right]\right| \leq \frac{2^{j-1} C_{1} \Delta x^{s+1-j}}{(j+1) ! \alpha^{j}}
$$

for $l=0,1, \ldots, p-j$. Case $j=1$ holds by (3.37). Use (3.34) to show that case $j$ implies case $j+1$. The derivative of the error function is

$$
\begin{aligned}
& \partial_{x} e_{i}^{n}(x)=e_{i}^{n}\left[x_{i-r-1 / 2}, x_{i-r+1 / 2}\right] \\
& \quad+\sum_{j=1}^{p} \sum_{\substack { q=0 \\
\begin{subarray}{c}{m=0 \\
m \neq q{ q = 0 \\
\begin{subarray} { c } { m = 0 \\
m \neq q } }\end{subarray}}^{j}\left(x-x_{i-r+m-1 / 2}\right) e_{i}^{n}\left[x_{i-r-1 / 2}, x_{i-r+1 / 2}, \ldots, x_{i-r+j+1 / 2}\right] .
\end{aligned}
$$

Insert (3.35), apply (3.38) with $l=0$ and use (3.22) to bound (3.39):

$$
\begin{aligned}
\left|\partial_{x} e_{i}^{n}(x)\right| \leq C_{0} \Delta x^{s}+\sum_{j=1}^{p} \sum_{q=0}^{j}(p & +1)^{j}(\Delta x)^{j} \frac{2^{j-1} C_{1} \Delta x^{s+1-j}}{(j+1) ! \alpha^{j}} \\
= & C_{0} \Delta x^{s}+C_{1} \Delta x^{s+1} \sum_{j=1}^{p}(p+1)^{j} \frac{2^{j-1}}{(j) ! \alpha^{j}} .
\end{aligned}
$$

Thus we have shown (3.31) in case $d=1$. Note that at the cell interface $x_{i+1 / 2}$,

$$
\begin{aligned}
& \left|\left[U\left(x_{i+1 / 2}, t^{n}\right)-\tilde{U}^{n}\left(x_{i+1 / 2}\right)\right]\right| \\
& \leq\left|U\left(x_{i+1 / 2}, t^{n}\right)-\partial_{x} q_{i+1}^{n}\left(x_{i+1 / 2}\right)\right|+\left|U\left(x_{i+1 / 2}, t^{n}\right)-\partial_{x} q_{i}^{n}\left(x_{i+1 / 2}\right)\right| \\
& +\left|\partial_{x}\left(q_{i+1}^{n}\left(x_{i+1 / 2}\right)-\tilde{q}_{i+1}^{n}\left(x_{i+1 / 2}\right)\right)-\partial_{x}\left(q_{i}^{n}\left(x_{i+1 / 2}\right)-\tilde{q}_{i}^{n}\left(x_{i+1 / 2}\right)\right)\right| \\
& =\left|U\left(x_{i+1 / 2}, t^{n}\right)-\partial_{x} q_{i+1}^{n}\left(x_{i+1 / 2}\right)\right|+\left|U\left(x_{i+1 / 2}, t^{n}\right)-\partial_{x} q_{i}^{n}\left(x_{i+1 / 2}\right)\right| \\
& +\left|\partial_{x} e_{i+1}^{n}\left(x_{i+1 / 2}\right)-\partial_{x} e_{i}^{n}\left(x_{i+1 / 2}\right)\right| \text {. }
\end{aligned}
$$


The last term on the right hand side of (3.41) is estimated using (3.39) and inserting (3.35):

$$
\begin{aligned}
& \left|\partial_{x} e_{i+1}^{n}\left(x_{i+1 / 2}\right)-\partial_{x} e_{i}^{n}\left(x_{i+1 / 2}\right)\right| \leq\left|\bar{e}_{i-r+1}^{n}-\bar{e}_{i-r}^{n}\right| \\
& +\left|\sum_{j=1}^{p} \sum_{q=0}^{j} \prod_{\substack{m=0 \\
m \neq q}}^{j}\left(x-x_{i-r+m+1 / 2}\right) e_{i+1}^{n}\left[x_{i-r+1 / 2}, x_{i-r+3 / 2}, \ldots, x_{i-r+j+3 / 2}\right]\right| \\
& +\left|\sum_{j=1}^{p} \sum_{q=0}^{j} \prod_{\substack{m=0 \\
m \neq q}}^{j}\left(x-x_{i-r+m-1 / 2}\right) e_{i}^{n}\left[x_{i-r-1 / 2}, x_{i-r+1 / 2}, \ldots, x_{i-r+j+1 / 2}\right]\right| .
\end{aligned}
$$

A rough bound analogous to (3.40) is found by inserting (3.23), from which it follows

$$
\left|\partial_{x} e_{i+1}^{n}\left(x_{i+1 / 2}\right)-\partial_{x} e_{i}^{n}\left(x_{i+1 / 2}\right)\right| \leq C_{1} \Delta x^{s+1} \sum_{j=0}^{p}(p+1)^{j} \frac{2^{j}}{(j) ! \alpha^{j}} .
$$

The result (3.28) follows from (3.41) and (3.43). The second derivative of the error function is

$$
\begin{aligned}
& \partial_{x}^{(2)} e_{i}^{n}(x)=2 e_{i}^{n}\left[x_{i-r-1 / 2}, x_{i-r+1 / 2}, x_{i-r+3 / 2}\right] \\
& +\sum_{j=2}^{p} \sum_{l=0}^{j} \sum_{\substack{q=0 \\
q \neq l}}^{j} \prod_{\substack{m=0 \\
m \neq q, l}}^{j}\left(x-x_{i-r+m-1 / 2}\right) e_{i}^{n}\left[x_{i-r-1 / 2}, x_{i-r+1 / 2}, \ldots, x_{i-r+j+1 / 2}\right] .
\end{aligned}
$$

It follows

$$
\left|\partial_{x}^{(2)} e_{i}^{n}(x)\right| \leq C_{1} \Delta x^{s} \sum_{j=1}^{p}(p+1)^{j-1} \frac{2^{j-1}}{(j-1) ! \alpha^{j}} .
$$

This proves (3.31) in case $d=2$. At a fixed $x \in \mathcal{K}_{i}^{h}$ for any $i=1,2, \ldots, M$ the time interpolation process is applied to define value in time on an arbitrary interval $\left[t^{n}, t^{n+1}\right]$. An analogous approach is used with (3.21), the CFL requirement and the triangle inequality to obtain the desired results (3.25)-(3.26). The result (3.27) follows from (3.24) and because the interpolant in time is of degree $p+1$.

3.2. Asymptotic properties of the computable error estimate. The rate at which the computable error estimate for a quantity of interest converges to the true error (see (3.15)) will be derived, given smooth data for the problems (3.1) and (3.11). The solution $u(x, t)$ of (3.1) is approximated using the reconstruction $\tilde{u}(x, t)$ and the solution $\phi(x, t)$ of (3.11) is approximated using the reconstruction $\tilde{\phi}(x, t)$ as defined in Section 3.1 above. The reconstructed solution $\tilde{u}$ consists of piece-wise polynomials of degree $p_{u}$, based on finite volume data $\bar{v}=\left\{\bar{v}_{i}^{n}\right\}$. The exact cell average data is $\left\{\bar{u}_{i}^{n}\right\}$ and the errors in the finite volume data are denoted by

$$
\left(\bar{e}_{u}\right)_{i}^{n}=\bar{u}_{i}^{n}-\bar{v}_{i}^{n} .
$$

The adjoint solution $\phi$ is reconstructed using piece-wise polynomials of degree $p_{\phi}$, based on finite volume data $\bar{w}=\left\{\bar{w}_{i}^{n}\right\}$. The exact cell average data is $\left\{\bar{\phi}_{i}^{n}\right\}$ and 
the errors in the finite volume data are denoted by

$$
\left(\bar{e}_{\phi}\right)_{i}^{n}=\bar{\phi}_{i}^{n}-\bar{w}_{i}^{n}
$$

Theorem 3.1. Let the solution $u(x, t)$ of $(3.1)$ and the solution $\phi(x, t)$ of $(3.11)$ be smooth. If the errors (3.46) satisfy (3.22) - (3.24) with $s=s_{u} \in \mathbb{N}$, and the errors (3.47) satisfy (3.22) - (3.24) with $s=s_{\phi} \in \mathbb{N}$, then under the assumptions of Lemma 3.1 it follows

$$
\begin{aligned}
e_{\mathcal{M}}(\tilde{u}) & =\tilde{e}_{\mathcal{M}}(\tilde{u}, \tilde{\phi})+\mathcal{O}\left(\Delta x^{q}\right) \\
q & =\min \left\{p_{u}+p_{\phi}+1, p_{u}+s_{\phi}+1, s_{u}+p_{\phi}, s_{u}+s_{\phi}\right\}
\end{aligned}
$$

Remark 3.2. The analysis could be extended easily to solutions in $H^{2}\left(\Omega_{T}\right)$ by looking at the order of approximation on functions in $C^{2}\left(\Omega_{T}\right)$. It is expected in this case that the minimum order of convergence will be $q=2$, e.g., if $u, \phi \in$ $H^{2}\left(\Omega_{T}\right) \backslash H^{3}\left(\Omega_{T}\right)$.

Remark 3.3. Since the error, computable error estimate and quantity of interest are all defined as integrals, and the reconstruction operators are designed to estimate the integral of the function being reconstructed, there are many opportunities to observe superconvergence in practice. Some examples are seen in Section 4.

Remark 3.4. In practice the choice of reconstruction polynomial should be chosen based upon the convergence rate of the finite volume schemes being used. The most efficient choice should normally be to set $p_{u} \geq s_{u}-1$ and $p_{\phi} \geq s_{\phi}$. This will be discussed further in Section 4.

Proof. It follows from (3.4), (3.14) and (3.15) that

$$
\begin{gathered}
3.50) e_{\mathcal{M}}(\tilde{u})-\tilde{e}_{\mathcal{M}}(\tilde{u}, \tilde{\phi})=A(u-\tilde{u}, \phi-\tilde{\phi})+\int_{\Omega}\left(u_{0}(x)-\tilde{u}(x, 0)\right)(\phi-\tilde{\phi})(x, 0) d x \\
=\sum_{i=1}^{M} \int_{\mathcal{K}_{i}^{h} \times(0, T)} \partial_{t}(u-\tilde{u})(\phi-\tilde{\phi}) d x d t-\sum_{i=1}^{M} \int_{\mathcal{K}_{i}^{h} \times(0, T)} a(u-\tilde{u}) \partial_{x}(\phi-\tilde{\phi}) d x d t \\
+\sum_{i=1}^{M} \int_{0}^{T} a(u-\tilde{u})_{-}\left(x_{i+1 / 2}\right)(\phi-\tilde{\phi})_{-}\left(x_{i+1 / 2}\right) d t \\
-\sum_{i=1}^{M} \int_{0}^{T} a(u-\tilde{u})_{-}\left(x_{i-1 / 2}\right)(\phi-\tilde{\phi})_{+}\left(x_{i-1 / 2}\right) d t \\
\quad+\sum_{i=1}^{M} \int_{\mathcal{K}_{i}^{h}}\left(u_{0}(x)-\tilde{u}(x, 0)\right)(\phi-\tilde{\phi})(x, 0) d x
\end{gathered}
$$


Due to the periodic boundary conditions, the terms evaluated at cell interfaces can be regrouped to yield

$$
e_{\mathcal{M}}(\tilde{u})-\tilde{e}_{\mathcal{M}}(\tilde{u}, \tilde{\phi})=\sum_{i=1}^{M} \int_{\mathcal{K}_{i}^{h} \times(0, T)} \partial_{t}(u-\tilde{u})(\phi-\tilde{\phi}) d x d t
$$

$$
\begin{aligned}
-\sum_{i=1}^{M} \int_{\mathcal{K}_{i}^{h} \times(0, T)} a(u-\tilde{u}) \partial_{x}(\phi-\tilde{\phi}) d x d t & -\sum_{i=1}^{M} \int_{0}^{T} a(u-\tilde{u})_{-}\left(x_{i+1 / 2}\right)\left[(\phi-\tilde{\phi})\left(x_{i+1 / 2}\right)\right] d t \\
& +\sum_{i=1}^{M} \int_{\mathcal{K}_{i}^{h}}\left(u_{0}(x)-\tilde{u}(x, 0)\right)(\phi-\tilde{\phi})(x, 0) d x .
\end{aligned}
$$

Proceeding term by term, (3.51) can be bounded using Lemma 3.1. The first term on the right hand side is bounded using (3.25) and (3.27):

$$
\begin{aligned}
& \left|\sum_{i=1}^{M} \int_{\mathcal{K}_{i}^{h} \times(0, T)} \partial_{t}(u-\tilde{u})(\phi-\tilde{\phi}) d x d t\right| \\
& \leq\left|\Omega_{T}\right|\left\|\partial_{t}(u-\tilde{u})\right\|_{L^{\infty}\left(\Omega_{T}\right)}\|\phi-\tilde{\phi}\|_{L^{\infty}\left(\Omega_{T}\right)} \\
& =\mathcal{O}\left(\left(\Delta x^{p_{u}+1}+\Delta x^{s_{u}}\right)\left(\Delta x^{p_{\phi}+1}+\Delta x^{s_{\phi}}\right)\right) .
\end{aligned}
$$

Similarly,

$$
\begin{aligned}
\left|\sum_{i=1}^{M} \int_{\mathcal{K}_{i}^{h} \times(0, T)} a(u-\tilde{u}) \partial_{x}(\phi-\tilde{\phi}) d x d t\right| & \\
& =\mathcal{O}\left(\left(\Delta x^{p_{u}+1}+\Delta x^{s_{u}}\right)\left(\Delta x^{p_{\phi}}+\Delta x^{s_{\phi}}\right)\right) .
\end{aligned}
$$

The next term on the right hand side of (3.51) satisfies

$$
\begin{aligned}
& \mid \sum_{i=1}^{M} \int_{0}^{T} a(u-\tilde{u})_{-}\left(x_{i+1 / 2}\right)\left[(\phi-\tilde{\phi})\left(x_{i+1 / 2}\right)\right] d t \mid \\
& \leq T M\left\{\max _{0 \leq t \leq T} \max _{i=1, \ldots, M}\left[(\phi-\tilde{\phi})\left(x_{i+1 / 2}, t\right)\right]\right\}\|u-\tilde{u}\|_{L^{\infty}\left(\Omega_{T}\right)} .
\end{aligned}
$$

Apply (3.28) and (3.21):

$$
\begin{aligned}
\left|\sum_{i=1}^{M} \int_{0}^{T} a(u-\tilde{u})_{-}\left(x_{i+1 / 2}\right)\left[(\phi-\tilde{\phi})\left(x_{i+1 / 2}\right)\right] d t\right| & \\
\leq \frac{\left|\Omega_{T}\right|}{\alpha \Delta x} \mathcal{O}\left(\left(\Delta x^{p_{u}+1}+\right.\right. & \left.\left.\Delta x^{s_{u}}\right)\left(\Delta x^{p_{\phi}+1}+\Delta x^{s_{\phi}+1}\right)\right) \\
& =\mathcal{O}\left(\left(\Delta x^{p_{u}+1}+\Delta x^{s_{u}}\right)\left(\Delta x^{p_{\phi}}+\Delta x^{s_{\phi}}\right)\right) .
\end{aligned}
$$

The last term on the right hand side of (3.51) satisfies

$$
\begin{aligned}
\mid \sum_{i=1}^{M} \int_{\mathcal{K}_{i}^{h}}\left(u_{0}(x)-\tilde{u}(x, 0)\right)(\phi & -\tilde{\phi})(x, 0) d x \mid \\
& =\mathcal{O}\left(\left(\Delta x^{p_{u}+1}+\Delta x^{s_{u}}\right)\left(\Delta x^{p_{\phi}+1}+\Delta x^{s_{\phi}}\right)\right) .
\end{aligned}
$$


It follows that

$$
e_{\mathcal{M}}(\tilde{u})-\tilde{e}_{\mathcal{M}}(\tilde{u}, \tilde{\phi})=\mathcal{O}\left(\Delta x^{p_{u}+s_{\phi}+1}+\Delta x^{s_{u}+s_{\phi}}+\Delta x^{s_{u}+p_{\phi}}+\Delta x^{p_{u}+p_{\phi}+1}\right),
$$

which is the desired result.

\section{Computational examples}

The various initial conditions, final time conditions, solver types and reconstruction types tested are described below. A system for labeling individual tests will be defined. The space-time domain is taken to be $[0,1] \times[0,1]$, and the advection coefficient is $a=1$. The initial conditions for the forward problem are denoted by $g_{j}(x)$ for $j=1,2, \ldots, 5$. In descending order of smoothness they are

$$
\begin{aligned}
g_{1}(x) & =\sin (2 \pi(x-1 / 4)) \\
\left(C^{7} \text {-pulse) } g_{2}(x)\right. & =\left\{\begin{aligned}
\cos ^{8}(2 \pi|x-0.5|), & \text { if }|x-0.5|<1 / 4 \\
0, & \text { otherwise }
\end{aligned}\right. \\
\left(C^{1} \text {-pulse) } g_{3}(x)\right. & =\left\{\begin{aligned}
\cos ^{2}(2 \pi|x-0.5|), & \text { if }|x-0.5|<1 / 4 \\
0, & \text { otherwise }
\end{aligned}\right. \\
\text { (triangle pulse) } g_{4}(x) & =\left\{\begin{aligned}
4 x-1, & \text { if } 1 / 4<x \leq 1 / 2 \\
3-4 x, & \text { if } 1 / 2<x \leq 3 / 4 \\
0, & \text { otherwise }
\end{aligned}\right. \\
\text { (square pulse) } g_{5}(x) & =\left\{\begin{aligned}
1, & \text { if }|x-0.5|<1 / 4 \\
0, & \text { otherwise. }
\end{aligned}\right.
\end{aligned}
$$

The quantities of interest are defined as final time conditions for the adjoint problem, denoted by $\psi_{j}(x)$ for $j=1,2, \ldots, 5$. Some are constructed by first defining a function centered at $x=0$, then recentering about $x^{*}=(1+\pi / 16) / 2 \in(1 / 2,1)$. This is done to avoid important points like maxima or the point of function evaluation to be located at any cell centers or interfaces. Specifically,

$$
\mathcal{M}(u)=\int_{0}^{1} \psi_{j}(x) u(x, t=1) d x
$$

for $j=1,2, \ldots, 6$, where

(4.6) (Fourier coefficient) $\psi_{1}(x)=\exp (-i 2 \pi x)$

(4.7) (evaluate at $\left.x=x^{*}\right) \psi_{2}(x)=\delta\left(x-x^{*}\right)$

(4.8) (moment, order 0) $\psi_{3}(x)=1$

(4.9) (moment, order 1) $\psi_{4}(x)=x$

(4.10) (moment, order 2) $\psi_{5}(x)=x^{2}$

$$
\begin{aligned}
\text { (solution energy) } \psi_{6}(x) & =\frac{1}{2} u(x, T) \\
\text { (patch average) } \psi_{7}(x) & =2, \text { if } 0.25<x<0.75, \text { o.w. } \phi_{7}(x)=0 .
\end{aligned}
$$

The sixth quantity of interest is nonlinear with respect to the forward solution and thus must be linearized. We follow the standard approach and linearize about the reconstructed solution $\tilde{u}$. Normally one computes a Fréchet derivative as part of the 
linearization process but for the solution energy some algebraic arguments suffice to derive the same approximation:

$$
\begin{aligned}
\mathcal{M}(u)-\mathcal{M}(\tilde{u}) & =\frac{1}{2} \int_{0}^{1}|u(x, T)|^{2} d x-\frac{1}{2} \int_{0}^{1}|\tilde{u}(x, T)|^{2} d x \\
& =\frac{1}{2} \int_{0}^{1}(u(x, T)+\tilde{u}(x, T))(u(x, T)-\tilde{u}(x, T)) d x \\
& \approx \int_{0}^{1} \tilde{u}(x, T)(u(x, T)-\tilde{u}(x, T)) d x .
\end{aligned}
$$

The adjoint data $\psi_{6}(x)$ is replaced by $\bar{\psi}_{6}(x)=\tilde{u}(x, T)$. The additional error committed by this approximation is

$$
\begin{aligned}
(\mathcal{M}(u)-\mathcal{M}(\tilde{u}))-\int_{0}^{1} \bar{\psi}_{6}(x)(u(x, T)- & \tilde{u}(x, T)) d x \\
& =\frac{1}{2} \int_{0}^{1}|u(x, T)-\tilde{u}(x, T)|^{2} d x .
\end{aligned}
$$

Three finite volume solver methods are employed. The first solver is the first order upwind scheme:

$$
\bar{u}_{j}^{n+1}=\bar{u}_{j}^{n}-a \frac{\Delta t}{\Delta x}\left(\bar{u}_{j}^{n}-\bar{u}_{j-1}^{n}\right) .
$$

The second solver is a second order upwind scheme. The scheme is derived by taking a piece-wise linear representation of the data on each cell $\mathcal{K}_{j}^{h}$ at time $t^{n}$ with cell average $\bar{u}_{j}^{n}$ and slope $\sigma_{j}^{n}$ defined below, then exactly solving the Riemann problem at each cell interface up to time $t^{n+1}$. The result is averaged over cell $\mathcal{K}_{j}^{h}$ to define $\bar{u}_{j}^{n+1}$. The update rule is derived by integrating the conservation law over $\mathcal{K}_{j}^{h} \times\left[t^{n}, t^{n+1}\right]$ and using the data on each cell interface at $x_{j+1 / 2}$ from the solution of the Riemann problem:

$$
\begin{aligned}
\bar{u}_{j}^{n+1} & =\bar{u}_{j}^{n}-a \frac{\Delta t}{\Delta x}\left(\bar{u}_{j}^{n}-\bar{u}_{j-1}^{n}+\frac{\Delta x-a \Delta t}{2}\left(\sigma_{j}^{n}-\sigma_{j-1}^{n}\right)\right), \\
\sigma_{j}^{n} & =\frac{\bar{u}_{j+1}^{n}-\bar{u}_{j-1}^{n}}{2 \Delta x} .
\end{aligned}
$$

The third solver is a high-fidelity, total variation diminishing (TVD) scheme. The scheme is derived the same way as second order upwind scheme, except that the slopes are chosen using the minimum modulus rule. The updates are given by

$$
\begin{aligned}
\bar{u}_{j}^{n+1} & =\bar{u}_{j}^{n}-a \frac{\Delta t}{\Delta x}\left(\bar{u}_{j}^{n}-\bar{u}_{j-1}^{n}+\frac{\Delta x-a \Delta t}{2}\left(\mu_{j}^{n}-\mu_{j-1}^{n}\right)\right), \\
\mu_{j}^{n} & =\frac{1}{\Delta x} \operatorname{MinMod}\left(\bar{u}_{j+1}^{n}-\bar{u}_{j}^{n}, \bar{u}_{j}^{n}-\bar{u}_{j-1}^{n}\right), \\
\operatorname{MinMod}\left(\eta_{1}, \eta_{2}\right) & =\left\{\begin{array}{cl}
\eta_{1}, & \text { if }\left|\eta_{1}\right|<\left|\eta_{2}\right|, \text { and } \eta_{1} \eta_{2}>0, \\
\eta_{2}, & \text { if }\left|\eta_{2}\right|<\left|\eta_{1}\right|, \text { and } \eta_{1} \eta_{2}>0, \\
0, & \text { otherwise. }
\end{array}\right.
\end{aligned}
$$

It is now possible to define a system of labeling the numerical tests. Let the approximation $\tilde{u}$ of the solution $u$ of (3.1) be constructed by taking finite volume data generated by one of the above methods and performing a space-time reconstruction of the data as described in Section 3.1. Given one of the quantities of 
TABLE 1. Convention for labeling initial conditions.

\begin{tabular}{|r|r|r|r|r|r|}
\hline Initial condition & IC1 & IC2 & IC3 & IC4 & IC5 \\
Equation & $(4.1)$ & $(4.2)$ & $(4.3)$ & $(4.4)$ & $(4.5)$ \\
\hline
\end{tabular}

TABLE 2. Convention for labeling quantities of interest.

\begin{tabular}{|r|r|r|r|r|r|r|r|}
\hline QOI & Q1 & Q2 & Q3 & Q4 & Q5 & Q6 & Q7 \\
Equation & $(4.6)$ & $(4.7)$ & $(4.8)$ & $(4.9)$ & $(4.10)$ & $(4.11)$ & $(4.12)$ \\
\hline
\end{tabular}

TABLE 3. Convention for labeling finite volume solvers.

\begin{tabular}{|r|r|r|r|}
\hline Label & FS1 or AS1 & FS2 or AS2 & FS3 or AS3 \\
Solver & order 1 upwind & order 2 upwind & high fidelity (MinMod) \\
\hline
\end{tabular}

interest implied by (4.6)-(4.11), let $\tilde{\phi}$ be constructed in an analogous way as an approximation to the solution $\phi$ of (3.11). The stencil shift index is taken to be $r=1$ (Section 3.1) in the tests, for both linear and quadratic reconstructions. Then there is an associated error in the quantity of interest, $e_{\mathcal{M}}(\tilde{u})$, and a computable estimate of this error, $\tilde{e}_{\mathcal{M}}(\tilde{u}, \tilde{\phi})$, as defined in (3.15). The accuracy of this error estimate will be denoted by

$$
\operatorname{acc}(\tilde{u}, \tilde{\phi})=e_{\mathcal{M}}(\tilde{u})-\tilde{e}_{\mathcal{M}}(\tilde{u}, \tilde{\phi}) .
$$

In each case there are six choices to be made:

- Initial conditions for (3.1), denoted by IC $k$ for $k=1,2, \ldots, 5$

- Terminal conditions for (3.11), denoted by $\mathrm{Q} k$ for $k=1,2, \ldots, 6$

- Forward finite volume solver, denoted by FS $k$ for $k=1,2,3$

- Adjoint finite volume solver, denoted by AS $k$ for $k=1,2,3$

- Spatial reconstruction, denoted by $\mathrm{R} k$ for $k=1,2$, for both forward and adjoint solutions.

Tables 1-3 makes the definitions precise. Each test is performed by first making these six choice and performing a convergence analysis for the quantities $e_{\mathcal{M}}(\tilde{u})$, $\tilde{e}_{\mathcal{M}}(\tilde{u}, \tilde{\phi})$ and $\operatorname{acc}(\tilde{u}, \tilde{\phi})$. The following example describes the labeling of the tests. The test label IC2-Q1-FS3R2-AS1R1 corresponds to choosing:

- Initial condition defined by (4.2)

- Adjoint terminal condition defined by (4.6)

- High-fidelity solver with piece-wise quadratic spatial reconstruction for the forward solution

- First order upwind solver with piece-wise linear spatial reconstruction for the forward solution

4.1. Fourier coefficient estimation. The results in this section pertain to the first non-constant Fourier coefficient quantity of interest, (4.6). This is an example where the solution of the adjoint problem is smooth. Then the results of Theorem 3.1 apply directly in this case for initial condition IC1 and also IC2, which is sufficiently smooth for the tests performed. Also, the theory can be extended to hold for IC3-IC4, understanding the convergence rate $q$ in Theorem 3.1 will be decreased according to the standard interpolation results with solutions in $H^{1}\left(\Omega_{T}\right)$. 
The convergence rate $q$ in Theorem 3.1 corresponds to the right-most convergence rate column in the tables.

The fifth initial condition, containing discontinuities, is included as computational evidence of how well the adjoint error estimation technique developed herein extends to the case of nonsmooth solutions. However, many methods of data reconstruction exist that address, in some form, the issue of discontinuities, e.g. [21], unlike the reconstructions used in the tests of this report. As an example, if the exact location (say $z$ ) of a discontinuity is known, one could define a grid with a cell interface at that point. The reconstruction stencil could be shifted for the cells on either side so as not to include the point $z$. Ways of identifying where discontinuities may be are possible using divided differences, as defined in Lemma 3.1. The application of such ideas should be explored in the current context.

4.1.1. Smooth initial condition with first order upwind. Tables 4-11 display the convergence results with a smooth forward and adjoint problem using the first-order upwind finite volume method for the forward problem. In Table 4 it is expected that $q=2$, as seen for the accuracy of the real part of the error estimate. A superconvergence result is observed for the imaginary part of the error. This case supports Theorem 3.1, as do all subsequent cases to which the theory applies directly. However, some important limitations of the theory are illuminated in the test cases. Though the imaginary part of the error is estimated reasonably well in Table 4, this could not be predicted since superconvergence results for $e_{\mathcal{M}}$ need not translate to similar results for $\operatorname{acc}(\tilde{u}, \tilde{\phi})$. We will return to this point later in Section 4.1.2.

In Tables 5, 6 and 7, combinations of first and second order reconstructions are employed for the adjoint and forward solution data. Since the solvers are both first order, the theory predicts that there need not be any appreciable increase in accuracy, as observed. Interestingly, in Table 8 the theory predicts $q=2$, while the values of $\operatorname{acc}(\tilde{u}, \tilde{\phi})$ are observed to converge at rate 3 , in spite of a seemingly suboptimal choice for the reconstruction of the adjoint data. It will be shown that this is not the case in general, also in Section 4.1.2.

The reconstruction of the adjoint data is quadratic in Table 9, resulting in an improvement in accuracy of the real part of the computable error estimate compared with Table 8. Table 10 is an example where the reconstruction of the adjoint data is theoretically suboptimal and the reconstruction of the forward solution is superoptimal. The theory is not violated in this case, but fails to predict the observed improvement in this case over test IC1 - Q1 - FS1 R1 - AS2 R1. The results in Table 11, perhaps surprisingly, do not yield a significant difference compared with tests IC1 - Q1 - FS1 R2 - AS2 R1 or IC1 - Q1 - FS1 R1 - AS2 R2. Even though the latter tests involve one linear and one quadratic reconstruction, both resulting in improvement over test IC1 - Q1 - FS1 R1 - AS2 R1, using quadratic reconstruction for both the forward and adjoint solutions yields no further improvement. 
TABle 4. Test: IC1 - Q1 - FS1 R1 - AS1 R1

\begin{tabular}{|r|r|rc|rc|rc|}
\hline Time steps & Cells & $\operatorname{Re}\left\{e_{\mathcal{M}}(\tilde{u})\right\}$ & Rate & $\operatorname{Re}\left\{\tilde{e}_{\mathcal{M}}(\tilde{u}, \tilde{\phi})\right\}$ & Rate & $\operatorname{Re}\{a c c(\tilde{u}, \tilde{\phi})\}$ & Rate \\
\hline 35 & 30 & $-4.4836 \mathrm{e}-02$ & & $-3.9500 \mathrm{e}-02$ & & $-5.3354 \mathrm{e}-03$ & \\
70 & 60 & $-2.2951 \mathrm{e}-02$ & 0.966 & $-2.1986 \mathrm{e}-02$ & 0.845 & $-9.6505 \mathrm{e}-04$ & 2.467 \\
140 & 120 & $-1.1612 \mathrm{e}-02$ & 0.983 & $-1.1421 \mathrm{e}-02$ & 0.945 & $-1.9098 \mathrm{e}-04$ & 2.337 \\
280 & 240 & $-5.8403 \mathrm{e}-03$ & 0.991 & $-5.7991 \mathrm{e}-03$ & 0.978 & $-4.1209 \mathrm{e}-05$ & 2.212 \\
560 & 480 & $-2.9288 \mathrm{e}-03$ & 0.996 & $-2.9193 \mathrm{e}-03$ & 0.990 & $-9.4703 \mathrm{e}-06$ & 2.121 \\
1120 & 960 & $-1.4665 \mathrm{e}-03$ & 0.998 & $-1.4643 \mathrm{e}-03$ & 0.995 & $-2.2626 \mathrm{e}-06$ & 2.065 \\
\hline \hline Time steps & Cells & $I m\left\{e_{\mathcal{M}}(\tilde{u})\right\}$ & Rate & $I m\left\{\tilde{e}_{\mathcal{M}}(\tilde{u}, \tilde{\phi})\right\}$ & Rate & $I m\{a c c(\tilde{u}, \tilde{\phi})\}$ & Rate \\
\hline 35 & 30 & $-2.3088 \mathrm{e}-03$ & & $-2.3810 \mathrm{e}-03$ & & $7.2240 \mathrm{e}-05$ & \\
70 & 60 & $-5.8195 \mathrm{e}-04$ & 1.988 & $-5.7316 \mathrm{e}-04$ & 2.055 & $-8.7861 \mathrm{e}-06$ & 3.039 \\
140 & 120 & $-1.4600 \mathrm{e}-04$ & 1.995 & $-1.4375 \mathrm{e}-04$ & 1.995 & $-2.2575 \mathrm{e}-06$ & 1.961 \\
280 & 240 & $-3.6562 \mathrm{e}-05$ & 1.998 & $-3.6206 \mathrm{e}-05$ & 1.989 & $-3.5586 \mathrm{e}-07$ & 2.665 \\
560 & 480 & $-9.1477 \mathrm{e}-06$ & 1.999 & $-9.0986 \mathrm{e}-06$ & 1.993 & $-4.9121 \mathrm{e}-08$ & 2.857 \\
1120 & 960 & $-2.2878 \mathrm{e}-06$ & 1.999 & $-2.2814 \mathrm{e}-06$ & 1.996 & $-6.4312 \mathrm{e}-09$ & 2.933 \\
\hline
\end{tabular}

TABLE 5. Test: IC1 - Q1 - FS1 R1 - AS1 R2

\begin{tabular}{|r|r|rc|rc|rc|}
\hline Time steps & Cells & $\operatorname{Re}\left\{e_{\mathcal{M}}(\tilde{u})\right\}$ & Rate & $\operatorname{Re}\left\{\tilde{e}_{\mathcal{M}}(\tilde{u}, \tilde{\phi})\right\}$ & Rate & $\operatorname{Re}\{a c c(\tilde{u}, \tilde{\phi})\}$ & Rate \\
\hline 35 & 30 & $-4.4836 \mathrm{e}-02$ & & $-4.2713 \mathrm{e}-02$ & & $-2.1226 \mathrm{e}-03$ & \\
70 & 60 & $-2.2951 \mathrm{e}-02$ & 0.966 & $-2.2415 \mathrm{e}-02$ & 0.930 & $-5.3646 \mathrm{e}-04$ & 1.984 \\
140 & 120 & $-1.1612 \mathrm{e}-02$ & 0.983 & $-1.1476 \mathrm{e}-02$ & 0.966 & $-1.3593 \mathrm{e}-04$ & 1.981 \\
280 & 240 & $-5.8403 \mathrm{e}-03$ & 0.991 & $-5.8061 \mathrm{e}-03$ & 0.983 & $-3.4243 \mathrm{e}-05$ & 1.989 \\
560 & 480 & $-2.9288 \mathrm{e}-03$ & 0.996 & $-2.9202 \mathrm{e}-03$ & 0.992 & $-8.5944 \mathrm{e}-06$ & 1.994 \\
1120 & 960 & $-1.4665 \mathrm{e}-03$ & 0.998 & $-1.4644 \mathrm{e}-03$ & 0.996 & $-2.1528 \mathrm{e}-06$ & 1.997 \\
\hline \hline Time steps & Cells & $I m\left\{e_{\mathcal{M}}(\tilde{u})\right\}$ & Rate & $I m\left\{\tilde{e}_{\mathcal{M}}(\tilde{u}, \tilde{\phi})\right\}$ & Rate & $\operatorname{Im}\{a c c(\tilde{u}, \tilde{\phi})\}$ & Rate \\
\hline 35 & 30 & $-2.3088 \mathrm{e}-03$ & & $-1.7847 \mathrm{e}-03$ & & $-5.2409 \mathrm{e}-04$ & \\
70 & 60 & $-5.8195 \mathrm{e}-04$ & 1.988 & $-5.3415 \mathrm{e}-04$ & 1.740 & $-4.7795 \mathrm{e}-05$ & 3.455 \\
140 & 120 & $-1.4600 \mathrm{e}-04$ & 1.995 & $-1.4126 \mathrm{e}-04$ & 1.919 & $-4.7437 \mathrm{e}-06$ & 3.333 \\
280 & 240 & $-3.6562 \mathrm{e}-05$ & 1.998 & $-3.6049 \mathrm{e}-05$ & 1.970 & $-5.1265 \mathrm{e}-07$ & 3.210 \\
560 & 480 & $-9.1477 \mathrm{e}-06$ & 1.999 & $-9.0887 \mathrm{e}-06$ & 1.988 & $-5.8963 \mathrm{e}-08$ & 3.120 \\
1120 & 960 & $-2.2878 \mathrm{e}-06$ & 1.999 & $-2.2808 \mathrm{e}-06$ & 1.995 & $-7.0475 \mathrm{e}-09$ & 3.065 \\
\hline
\end{tabular}


TABle 6. Test: IC1 - Q1 - FS1 R2 - AS1 R1

\begin{tabular}{|r|r|rc|rc|rc|}
\hline Time steps & Cells & $\operatorname{Re}\left\{e_{\mathcal{M}}(\tilde{u})\right\}$ & Rate & $\operatorname{Re}\left\{\tilde{e}_{\mathcal{M}}(\tilde{u}, \tilde{\phi})\right\}$ & Rate & $\operatorname{Re}\{a c c(\tilde{u}, \tilde{\phi})\}$ & Rate \\
\hline 35 & 30 & $-4.4834 \mathrm{e}-02$ & & $-4.2767 \mathrm{e}-02$ & & $-2.0667 \mathrm{e}-03$ & \\
70 & 60 & $-2.2951 \mathrm{e}-02$ & 0.966 & $-2.2417 \mathrm{e}-02$ & 0.932 & $-5.3465 \mathrm{e}-04$ & 1.951 \\
140 & 120 & $-1.1612 \mathrm{e}-02$ & 0.983 & $-1.1476 \mathrm{e}-02$ & 0.966 & $-1.3587 \mathrm{e}-04$ & 1.976 \\
280 & 240 & $-5.8403 \mathrm{e}-03$ & 0.991 & $-5.8061 \mathrm{e}-03$ & 0.983 & $-3.4241 \mathrm{e}-05$ & 1.988 \\
560 & 480 & $-2.9288 \mathrm{e}-03$ & 0.996 & $-2.9202 \mathrm{e}-03$ & 0.992 & $-8.5943 \mathrm{e}-06$ & 1.994 \\
1120 & 960 & $-1.4665 \mathrm{e}-03$ & 0.998 & $-1.4644 \mathrm{e}-03$ & 0.996 & $-2.1528 \mathrm{e}-06$ & 1.997 \\
\hline \hline Time steps & Cells & $I m\left\{e_{\mathcal{M}}(\tilde{u})\right\}$ & Rate & $I m\left\{\tilde{e}_{\mathcal{M}}(\tilde{u}, \tilde{\phi})\right\}$ & Rate & $I m\{a c c(\tilde{u}, \tilde{\phi})\}$ & Rate \\
\hline 35 & 30 & $-2.1357 \mathrm{e}-03$ & & $-2.0966 \mathrm{e}-03$ & & $-3.9058 \mathrm{e}-05$ & \\
70 & 60 & $-5.5916 \mathrm{e}-04$ & 1.933 & $-5.4349 \mathrm{e}-04$ & 1.948 & $-1.5667 \mathrm{e}-05$ & 1.318 \\
140 & 120 & $-1.4308 \mathrm{e}-04$ & 1.966 & $-1.4040 \mathrm{e}-04$ & 1.953 & $-2.6838 \mathrm{e}-06$ & 2.545 \\
280 & 240 & $-3.6192 \mathrm{e}-05$ & 1.983 & $-3.5810 \mathrm{e}-05$ & 1.971 & $-3.8236 \mathrm{e}-07$ & 2.811 \\
560 & 480 & $-9.1012 \mathrm{e}-06$ & 1.992 & $-9.0505 \mathrm{e}-06$ & 1.984 & $-5.0773 \mathrm{e}-08$ & 2.913 \\
1120 & 960 & $-2.2820 \mathrm{e}-06$ & 1.996 & $-2.2755 \mathrm{e}-06$ & 1.992 & $-6.5343 \mathrm{e}-09$ & 2.958 \\
\hline
\end{tabular}

TABle 7. Test: IC1 - Q1 - FS1 R2 - AS1 R2

\begin{tabular}{|r|r|rc|rc|rr|}
\hline Time steps & Cells & $\operatorname{Re}\left\{e_{\mathcal{M}}(\tilde{u})\right\}$ & Rate & $\operatorname{Re}\left\{\tilde{e}_{\mathcal{M}}(\tilde{u}, \tilde{\phi})\right\}$ & Rate & $\operatorname{Re}\{a c c(\tilde{u}, \tilde{\phi})\}$ & Rate \\
\hline 35 & 30 & $-4.4834 \mathrm{e}-02$ & & $-4.2747 \mathrm{e}-02$ & & $-2.0862 \mathrm{e}-03$ & \\
70 & 60 & $-2.2951 \mathrm{e}-02$ & 0.966 & $-2.2416 \mathrm{e}-02$ & 0.931 & $-5.3528 \mathrm{e}-04$ & 1.963 \\
140 & 120 & $-1.1612 \mathrm{e}-02$ & 0.983 & $-1.1476 \mathrm{e}-02$ & 0.966 & $-1.3589 \mathrm{e}-04$ & 1.978 \\
280 & 240 & $-5.8403 \mathrm{e}-03$ & 0.991 & $-5.8061 \mathrm{e}-03$ & 0.983 & $-3.4242 \mathrm{e}-05$ & 1.989 \\
560 & 480 & $-2.9288 \mathrm{e}-03$ & 0.996 & $-2.9202 \mathrm{e}-03$ & 0.992 & $-8.5944 \mathrm{e}-06$ & 1.994 \\
1120 & 960 & $-1.4665 \mathrm{e}-03$ & 0.998 & $-1.4644 \mathrm{e}-03$ & 0.996 & $-2.1528 \mathrm{e}-06$ & 1.997 \\
\hline \hline Time steps & Cells & $I m\left\{e_{\mathcal{M}}(\tilde{u})\right\}$ & Rate & $I m\left\{\tilde{e}_{\mathcal{M}}(\tilde{u}, \tilde{\phi})\right\}$ & Rate & $I m\{a c c(\tilde{u}, \tilde{\phi})\}$ & Rate \\
\hline 35 & 30 & $-2.1357 \mathrm{e}-03$ & & $-1.9367 \mathrm{e}-03$ & & $-1.9900 \mathrm{e}-04$ & \\
70 & 60 & $-5.5916 \mathrm{e}-04$ & 1.933 & $-5.3291 \mathrm{e}-04$ & 1.862 & $-2.6248 \mathrm{e}-05$ & 2.923 \\
140 & 120 & $-1.4308 \mathrm{e}-04$ & 1.966 & $-1.3972 \mathrm{e}-04$ & 1.931 & $-3.3618 \mathrm{e}-06$ & 2.965 \\
280 & 240 & $-3.6192 \mathrm{e}-05$ & 1.983 & $-3.5767 \mathrm{e}-05$ & 1.966 & $-4.2523 \mathrm{e}-07$ & 2.983 \\
560 & 480 & $-9.1012 \mathrm{e}-06$ & 1.992 & $-9.0478 \mathrm{e}-06$ & 1.983 & $-5.3467 \mathrm{e}-08$ & 2.992 \\
1120 & 960 & $-2.2820 \mathrm{e}-06$ & 1.996 & $-2.2753 \mathrm{e}-06$ & 1.992 & $-6.7031 \mathrm{e}-09$ & 2.996 \\
\hline
\end{tabular}


TABle 8. Test: IC1 - Q1 - FS1 R1 - AS2 R1

\begin{tabular}{|r|r|rc|rc|rc|}
\hline Time steps & Cells & $\operatorname{Re}\left\{e_{\mathcal{M}}(\tilde{u})\right\}$ & Rate & $\operatorname{Re}\left\{\tilde{e}_{\mathcal{M}}(\tilde{u}, \tilde{\phi})\right\}$ & Rate & $\operatorname{Re}\{a c c(\tilde{u}, \tilde{\phi})\}$ & Rate \\
\hline 35 & 30 & $-4.4836 \mathrm{e}-02$ & & $-4.1386 \mathrm{e}-02$ & & $-3.4497 \mathrm{e}-03$ & \\
70 & 60 & $-2.2951 \mathrm{e}-02$ & 0.966 & $-2.2509 \mathrm{e}-02$ & 0.879 & $-4.4215 \mathrm{e}-04$ & 2.964 \\
140 & 120 & $-1.1612 \mathrm{e}-02$ & 0.983 & $-1.1556 \mathrm{e}-02$ & 0.962 & $-5.5853 \mathrm{e}-05$ & 2.985 \\
280 & 240 & $-5.8403 \mathrm{e}-03$ & 0.991 & $-5.8333 \mathrm{e}-03$ & 0.986 & $-7.0150 \mathrm{e}-06$ & 2.993 \\
560 & 480 & $-2.9288 \mathrm{e}-03$ & 0.996 & $-2.9279 \mathrm{e}-03$ & 0.994 & $-8.7887 \mathrm{e}-07$ & 2.997 \\
1120 & 960 & $-1.4665 \mathrm{e}-03$ & 0.998 & $-1.4664 \mathrm{e}-03$ & 0.998 & $-1.0998 \mathrm{e}-07$ & 2.998 \\
\hline \hline Time steps & Cells & $I m\left\{e_{\mathcal{M}}(\tilde{u})\right\}$ & Rate & $I m\left\{\tilde{e}_{\mathcal{M}}(\tilde{u}, \tilde{\phi})\right\}$ & Rate & $\operatorname{Im}\{a c c(\tilde{u}, \tilde{\phi})\}$ & Rate \\
\hline 35 & 30 & $-2.3088 \mathrm{e}-03$ & & $-2.6503 \mathrm{e}-03$ & & $3.4154 \mathrm{e}-04$ & \\
70 & 60 & $-5.8195 \mathrm{e}-04$ & 1.988 & $-6.0725 \mathrm{e}-04$ & 2.126 & $2.5306 \mathrm{e}-05$ & 3.755 \\
140 & 120 & $-1.4600 \mathrm{e}-04$ & 1.995 & $-1.4803 \mathrm{e}-04$ & 2.036 & $2.0261 \mathrm{e}-06$ & 3.643 \\
280 & 240 & $-3.6562 \mathrm{e}-05$ & 1.998 & $-3.6742 \mathrm{e}-05$ & 2.010 & $1.8083 \mathrm{e}-07$ & 3.486 \\
560 & 480 & $-9.1477 \mathrm{e}-06$ & 1.999 & $-9.1657 \mathrm{e}-06$ & 2.003 & $1.8037 \mathrm{e}-08$ & 3.326 \\
1120 & 960 & $-2.2878 \mathrm{e}-06$ & 1.999 & $-2.2898 \mathrm{e}-06$ & 2.001 & $1.9681 \mathrm{e}-09$ & 3.196 \\
\hline
\end{tabular}

TABle 9. Test: IC1 - Q1 - FS1 R1 - AS2 R2

\begin{tabular}{|r|r|rc|rc|rc|}
\hline Time steps & Cells & $\operatorname{Re}\left\{e_{\mathcal{M}}(\tilde{u})\right\}$ & Rate & $\operatorname{Re}\left\{\tilde{e}_{\mathcal{M}}(\tilde{u}, \tilde{\phi})\right\}$ & Rate & $\operatorname{Re}\{a c c(\tilde{u}, \tilde{\phi})\}$ & Rate \\
\hline 35 & 30 & $-4.4836 \mathrm{e}-02$ & & $-4.4756 \mathrm{e}-02$ & & $-7.9588 \mathrm{e}-05$ & \\
70 & 60 & $-2.2951 \mathrm{e}-02$ & 0.966 & $-2.2948 \mathrm{e}-02$ & 0.964 & $-3.2952 \mathrm{e}-06$ & 4.594 \\
140 & 120 & $-1.1612 \mathrm{e}-02$ & 0.983 & $-1.1612 \mathrm{e}-02$ & 0.983 & $-1.5063 \mathrm{e}-07$ & 4.451 \\
280 & 240 & $-5.8403 \mathrm{e}-03$ & 0.991 & $-5.8403 \mathrm{e}-03$ & 0.991 & $-7.6481 \mathrm{e}-09$ & 4.300 \\
560 & 480 & $-2.9288 \mathrm{e}-03$ & 0.996 & $-2.9288 \mathrm{e}-03$ & 0.996 & $-4.2232 \mathrm{e}-10$ & 4.179 \\
1120 & 960 & $-1.4665 \mathrm{e}-03$ & 0.998 & $-1.4665 \mathrm{e}-03$ & 0.998 & $-2.4660 \mathrm{e}-11$ & 4.098 \\
\hline \hline Time steps & Cells & $I m\left\{e_{\mathcal{M}}(\tilde{u})\right\}$ & Rate & $I m\left\{\tilde{e}_{\mathcal{M}}(\tilde{u}, \tilde{\phi})\right\}$ & Rate & $I m\{a c c(\tilde{u}, \tilde{\phi})\}$ & Rate \\
\hline 35 & 30 & $-2.3088 \mathrm{e}-03$ & & $-2.0373 \mathrm{e}-03$ & & $-2.7147 \mathrm{e}-04$ & \\
70 & 60 & $-5.8195 \mathrm{e}-04$ & 1.988 & $-5.6770 \mathrm{e}-04$ & 1.843 & $-1.4244 \mathrm{e}-05$ & 4.252 \\
140 & 120 & $-1.4600 \mathrm{e}-04$ & 1.995 & $-1.4553 \mathrm{e}-04$ & 1.964 & $-4.7721 \mathrm{e}-07$ & 4.900 \\
280 & 240 & $-3.6562 \mathrm{e}-05$ & 1.998 & $-3.6585 \mathrm{e}-05$ & 1.992 & $2.3503 \mathrm{e}-08$ & 4.344 \\
560 & 480 & $-9.1477 \mathrm{e}-06$ & 1.999 & $-9.1559 \mathrm{e}-06$ & 1.998 & $8.1789 \mathrm{e}-09$ & 1.523 \\
1120 & 960 & $-2.2878 \mathrm{e}-06$ & 1.999 & $-2.2892 \mathrm{e}-06$ & 2.000 & $1.3511 \mathrm{e}-09$ & 2.598 \\
\hline
\end{tabular}


TABLE 10. Test: IC1 - Q1 - FS1 R2 - AS2 R1

\begin{tabular}{|r|r|rc|rc|rc|}
\hline Time steps & Cells & $\operatorname{Re}\left\{e_{\mathcal{M}}(\tilde{u})\right\}$ & Rate & $\operatorname{Re}\left\{\tilde{e}_{\mathcal{M}}(\tilde{u}, \tilde{\phi})\right\}$ & Rate & $\operatorname{Re}\{a c c(\tilde{u}, \tilde{\phi})\}$ & Rate \\
\hline 35 & 30 & $-4.4834 \mathrm{e}-02$ & & $-4.4812 \mathrm{e}-02$ & & $-2.1677 \mathrm{e}-05$ & \\
70 & 60 & $-2.2951 \mathrm{e}-02$ & 0.966 & $-2.2950 \mathrm{e}-02$ & 0.965 & $-1.4316 \mathrm{e}-06$ & 3.920 \\
140 & 120 & $-1.1612 \mathrm{e}-02$ & 0.983 & $-1.1612 \mathrm{e}-02$ & 0.983 & $-9.1689 \mathrm{e}-08$ & 3.965 \\
280 & 240 & $-5.8403 \mathrm{e}-03$ & 0.991 & $-5.8403 \mathrm{e}-03$ & 0.991 & $-5.7965 \mathrm{e}-09$ & 3.983 \\
560 & 480 & $-2.9288 \mathrm{e}-03$ & 0.996 & $-2.9288 \mathrm{e}-03$ & 0.996 & $-3.6429 \mathrm{e}-10$ & 3.992 \\
1120 & 960 & $-1.4665 \mathrm{e}-03$ & 0.998 & $-1.4665 \mathrm{e}-03$ & 0.998 & $-2.2840 \mathrm{e}-11$ & 3.995 \\
\hline \hline Time steps & Cells & $I m\left\{e_{\mathcal{M}}(\tilde{u})\right\}$ & Rate & $I m\left\{\tilde{e}_{\mathcal{M}}(\tilde{u}, \tilde{\phi})\right\}$ & Rate & $I m\{a c c(\tilde{u}, \tilde{\phi})\}$ & Rate \\
\hline 35 & 30 & $-2.1357 \mathrm{e}-03$ & & $-2.3560 \mathrm{e}-03$ & & $2.2029 \mathrm{e}-04$ & \\
70 & 60 & $-5.5916 \mathrm{e}-04$ & 1.933 & $-5.7672 \mathrm{e}-04$ & 2.030 & $1.7559 \mathrm{e}-05$ & 3.649 \\
140 & 120 & $-1.4308 \mathrm{e}-04$ & 1.966 & $-1.4462 \mathrm{e}-04$ & 1.996 & $1.5377 \mathrm{e}-06$ & 3.513 \\
280 & 240 & $-3.6192 \mathrm{e}-05$ & 1.983 & $-3.6342 \mathrm{e}-05$ & 1.993 & $1.5019 \mathrm{e}-07$ & 3.356 \\
560 & 480 & $-9.1012 \mathrm{e}-06$ & 1.992 & $-9.1174 \mathrm{e}-06$ & 1.995 & $1.6119 \mathrm{e}-08$ & 3.220 \\
1120 & 960 & $-2.2820 \mathrm{e}-06$ & 1.996 & $-2.2838 \mathrm{e}-06$ & 1.997 & $1.8481 \mathrm{e}-09$ & 3.125 \\
\hline
\end{tabular}

TABle 11. Test: IC1 - Q1 - FS1 R2 - AS2 R2

\begin{tabular}{|r|r|rc|rc|rr|}
\hline Time steps & Cells & $\operatorname{Re}\left\{e_{\mathcal{M}}(\tilde{u})\right\}$ & Rate & $\operatorname{Re}\left\{\tilde{e}_{\mathcal{M}}(\tilde{u}, \tilde{\phi})\right\}$ & Rate & $\operatorname{Re}\{a c c(\tilde{u}, \tilde{\phi})\}$ & Rate \\
\hline 35 & 30 & $-4.4834 \mathrm{e}-02$ & & $-4.4792 \mathrm{e}-02$ & & $-4.1579 \mathrm{e}-05$ & \\
70 & 60 & $-2.2951 \mathrm{e}-02$ & 0.966 & $-2.2949 \mathrm{e}-02$ & 0.965 & $-2.0721 \mathrm{e}-06$ & 4.327 \\
140 & 120 & $-1.1612 \mathrm{e}-02$ & 0.983 & $-1.1612 \mathrm{e}-02$ & 0.983 & $-1.1195 \mathrm{e}-07$ & 4.210 \\
280 & 240 & $-5.8403 \mathrm{e}-03$ & 0.991 & $-5.8403 \mathrm{e}-03$ & 0.991 & $-6.4329 \mathrm{e}-09$ & 4.121 \\
560 & 480 & $-2.9288 \mathrm{e}-03$ & 0.996 & $-2.9288 \mathrm{e}-03$ & 0.996 & $-3.8419 \mathrm{e}-10$ & 4.066 \\
1120 & 960 & $-1.4665 \mathrm{e}-03$ & 0.998 & $-1.4665 \mathrm{e}-03$ & 0.998 & $-2.3393 \mathrm{e}-11$ & 4.038 \\
\hline \hline Time steps & Cells & $I m\left\{e_{\mathcal{M}}(\tilde{u})\right\}$ & Rate & $\operatorname{Im}\left\{\tilde{e}_{\mathcal{M}}(\tilde{u}, \tilde{\phi})\right\}$ & Rate & $\operatorname{Im}\{a c c(\tilde{u}, \tilde{\phi})\}$ & Rate \\
\hline 35 & 30 & $-2.1357 \mathrm{e}-03$ & & $-2.1882 \mathrm{e}-03$ & & $5.2532 \mathrm{e}-05$ & \\
70 & 60 & $-5.5916 \mathrm{e}-04$ & 1.933 & $-5.6588 \mathrm{e}-04$ & 1.951 & $6.7242 \mathrm{e}-06$ & 2.966 \\
140 & 120 & $-1.4308 \mathrm{e}-04$ & 1.966 & $-1.4394 \mathrm{e}-04$ & 1.975 & $8.5172 \mathrm{e}-07$ & 2.981 \\
280 & 240 & $-3.6192 \mathrm{e}-05$ & 1.983 & $-3.6299 \mathrm{e}-05$ & 1.987 & $1.0707 \mathrm{e}-07$ & 2.992 \\
560 & 480 & $-9.1012 \mathrm{e}-06$ & 1.992 & $-9.1146 \mathrm{e}-06$ & 1.994 & $1.3417 \mathrm{e}-08$ & 2.996 \\
1120 & 960 & $-2.2820 \mathrm{e}-06$ & 1.996 & $-2.2837 \mathrm{e}-06$ & 1.997 & $1.6790 \mathrm{e}-09$ & 2.998 \\
\hline
\end{tabular}

4.1.2. Smooth initial condition with second order upwind. The second order upwind finite volume method was applied for the tests represented in Tables $12-19$. The test IC1 - Q1 - FS2 R1 - AS1 R1 in Table 12 is an important example where the accuracy of the real part of the computable error estimate is converging at the rate 3 , consistent with Theorem 3.1, but the accuracy is poor relative to the size of the true error. The theory would be strengthened if a method were to be developed with a provable estimate of the form

$$
\left|e_{\mathcal{M}}(\tilde{u})-\tilde{e}_{\mathcal{M}}(\tilde{u}, \tilde{\phi})\right| \leq C \Delta x^{q}\left|e_{\mathcal{M}}(\tilde{u})\right| .
$$

In the a posteriori literature for elliptic type problems there is a measurement of quality for the computable error estimate known at the effectivity index, (see e.g. [1]), being the size of the ratio of the computable error estimate to the true 
error. More precisely, if $\epsilon$ is the effectivity index, then

$$
\epsilon=\frac{\left|\tilde{e}_{\mathcal{M}}(\tilde{u}, \tilde{\phi})\right|}{\left|e_{\mathcal{M}}(\tilde{u})\right|} .
$$

It is desirable to be able to say $\epsilon \rightarrow 1$ asymptotically. Clearly, this would follow from the result (4.22). Such a result is not currently known to exist in the literature for hyperbolic conservation laws. However, test IC1 - Q1 - FS2 R1 - AS1 R1 does not represent a cause for concern in practice, since the reconstruction of the forward solution chosen is suboptimal. This is fixed in test IC1 - Q1 - FS2 R2 - AS1 R1, as seen in Table 14, and the accuracy is improved. Similar improvement is also observed in Tables 13 and 15.

The improvement in the real part of the error estimate of Table 13 compared to Table 12 is unexpected and not understood, as it is not clear why a higher order reconstruction of data from a first order finite volume method should yield an improvement in accuracy. It may be related to the fact that the quantity of interest and computable error estimate all take the form of integrals, and the reconstruction operation is based on approximating the integral of the function being reconstructed. There could be some cancellation of error related to integration taken advantage of by the higher order reconstruction.

In Tables 16 - 19 both finite volume solvers are second order. A phenomenon is observed similar to that of Tables 12 - 15, where the test with only linear reconstructions agrees with Theorem 3.1, but fails to yield an error estimate with a relative accuracy that is decreasing with the grid size. Upon using quadratic reconstruction for either the forward or adjoint data, the result is greatly improved. In Tables 17 - 19 the convergence rates for the accuracy are higher than the lower bound predicted by Theorem 3.1. A difference with this set of tests versus the former set using a first order adjoint solver is that using quadratic reconstruction for both the forward and adjoint data is seen to yield the best accuracy on the finest grid. This implies that, in the terminology of Theorem 3.1, one should choose reconstruction operators to follow the rule $p_{u} \geq s_{u}$ and $p_{\phi} \geq s_{\phi}$.

TABLE 12. Test: IC1 - Q1 - FS2 R1 - AS1 R1

\begin{tabular}{|r|r|rc|rc|rr|}
\hline Time steps & Cells & $\operatorname{Re}\left\{e_{\mathcal{M}}(\tilde{u})\right\}$ & Rate & $\operatorname{Re}\left\{\tilde{e}_{\mathcal{M}}(\tilde{u}, \tilde{\phi})\right\}$ & Rate & $\operatorname{Re}\{a c c(\tilde{u}, \tilde{\phi})\}$ & Rate \\
\hline 35 & 30 & $-4.6629 \mathrm{e}-04$ & & $2.9809 \mathrm{e}-03$ & & $-3.4472 \mathrm{e}-03$ & \\
70 & 60 & $-5.7522 \mathrm{e}-05$ & 3.019 & $3.8455 \mathrm{e}-04$ & 2.954 & $-4.4207 \mathrm{e}-04$ & 2.963 \\
140 & 120 & $-7.1318 \mathrm{e}-06$ & 3.012 & $4.8719 \mathrm{e}-05$ & 2.981 & $-5.5851 \mathrm{e}-05$ & 2.985 \\
280 & 240 & $-8.8752 \mathrm{e}-07$ & 3.006 & $6.1274 \mathrm{e}-06$ & 2.991 & $-7.0150 \mathrm{e}-06$ & 2.993 \\
560 & 480 & $-1.1068 \mathrm{e}-07$ & 3.003 & $7.6818 \mathrm{e}-07$ & 2.996 & $-8.7887 \mathrm{e}-07$ & 2.997 \\
1120 & 960 & $-1.3819 \mathrm{e}-08$ & 3.002 & $9.6161 \mathrm{e}-08$ & 2.998 & $-1.0998 \mathrm{e}-07$ & 2.998 \\
\hline \hline Time steps & Cells & $I m\left\{e_{\mathcal{M}}(\tilde{u})\right\}$ & Rate & $\operatorname{Im}\left\{\tilde{e}_{\mathcal{M}}(\tilde{u}, \tilde{\phi})\right\}$ & Rate & $\operatorname{Im}\{a c c(\tilde{u}, \tilde{\phi})\}$ & Rate \\
\hline 35 & 30 & $9.4943 \mathrm{e}-04$ & & $6.4163 \mathrm{e}-04$ & & $3.0780 \mathrm{e}-04$ & \\
70 & 60 & $2.6707 \mathrm{e}-04$ & 1.830 & $2.4395 \mathrm{e}-04$ & 1.395 & $2.3118 \mathrm{e}-05$ & 3.735 \\
140 & 120 & $7.0125 \mathrm{e}-05$ & 1.929 & $6.8238 \mathrm{e}-05$ & 1.838 & $1.8874 \mathrm{e}-06$ & 3.615 \\
280 & 240 & $1.7928 \mathrm{e}-05$ & 1.968 & $1.7756 \mathrm{e}-05$ & 1.942 & $1.7210 \mathrm{e}-07$ & 3.455 \\
560 & 480 & $4.5302 \mathrm{e}-06$ & 1.985 & $4.5127 \mathrm{e}-06$ & 1.976 & $1.7490 \mathrm{e}-08$ & 3.299 \\
1120 & 960 & $1.1385 \mathrm{e}-06$ & 1.992 & $1.1365 \mathrm{e}-06$ & 1.989 & $1.9338 \mathrm{e}-09$ & 3.177 \\
\hline
\end{tabular}


TABle 13. Test: IC1 - Q1 - FS2 R1 - AS1 R2

\begin{tabular}{|r|r|rc|rc|rr|}
\hline Time steps & Cells & $\operatorname{Re}\left\{e_{\mathcal{M}}(\tilde{u})\right\}$ & Rate & $\operatorname{Re}\left\{\tilde{e}_{\mathcal{M}}(\tilde{u}, \tilde{\phi})\right\}$ & Rate & $\operatorname{Re}\{a c c(\tilde{u}, \tilde{\phi})\}$ & Rate \\
\hline 35 & 30 & $-4.6629 \mathrm{e}-04$ & & $-3.8783 \mathrm{e}-04$ & & $-7.8461 \mathrm{e}-05$ & \\
70 & 60 & $-5.7522 \mathrm{e}-05$ & 3.019 & $-5.4264 \mathrm{e}-05$ & 2.837 & $-3.2584 \mathrm{e}-06$ & 4.590 \\
140 & 120 & $-7.1318 \mathrm{e}-06$ & 3.012 & $-6.9824 \mathrm{e}-06$ & 2.958 & $-1.4946 \mathrm{e}-07$ & 4.446 \\
280 & 240 & $-8.8752 \mathrm{e}-07$ & 3.006 & $-8.7991 \mathrm{e}-07$ & 2.988 & $-7.6112 \mathrm{e}-09$ & 4.295 \\
560 & 480 & $-1.1068 \mathrm{e}-07$ & 3.003 & $-1.1026 \mathrm{e}-07$ & 2.996 & $-4.2116 \mathrm{e}-10$ & 4.176 \\
1120 & 960 & $-1.3819 \mathrm{e}-08$ & 3.002 & $-1.3794 \mathrm{e}-08$ & 2.999 & $-2.4625 \mathrm{e}-11$ & 4.096 \\
\hline \hline Time steps & Cells & $I m\left\{e_{\mathcal{M}}(\tilde{u})\right\}$ & Rate & $I m\left\{\tilde{e}_{\mathcal{M}}(\tilde{u}, \tilde{\phi})\right\}$ & Rate & $I m\{a c c(\tilde{u}, \tilde{\phi})\}$ & Rate \\
\hline 35 & 30 & $9.4943 \mathrm{e}-04$ & & $1.2378 \mathrm{e}-03$ & & $-2.8835 \mathrm{e}-04$ & \\
70 & 60 & $2.6707 \mathrm{e}-04$ & 1.830 & $2.8241 \mathrm{e}-04$ & 2.132 & $-1.5338 \mathrm{e}-05$ & 4.233 \\
140 & 120 & $7.0125 \mathrm{e}-05$ & 1.929 & $7.0672 \mathrm{e}-05$ & 1.999 & $-5.4659 \mathrm{e}-07$ & 4.810 \\
280 & 240 & $1.7928 \mathrm{e}-05$ & 1.968 & $1.7909 \mathrm{e}-05$ & 1.980 & $1.9137 \mathrm{e}-08$ & 4.836 \\
560 & 480 & $4.5302 \mathrm{e}-06$ & 1.985 & $4.5223 \mathrm{e}-06$ & 1.986 & $7.9052 \mathrm{e}-09$ & 1.276 \\
1120 & 960 & $1.1385 \mathrm{e}-06$ & 1.992 & $1.1371 \mathrm{e}-06$ & 1.992 & $1.3340 \mathrm{e}-09$ & 2.567 \\
\hline
\end{tabular}

TABle 14. Test: IC1 - Q1 - FS2 R2 - AS1 R1

\begin{tabular}{|r|r|rc|rc|rr|}
\hline Time steps & Cells & $\operatorname{Re}\left\{e_{\mathcal{M}}(\tilde{u})\right\}$ & Rate & $\operatorname{Re}\left\{\tilde{e}_{\mathcal{M}}(\tilde{u}, \tilde{\phi})\right\}$ & Rate & $\operatorname{Re}\{a c c(\tilde{u}, \tilde{\phi})\}$ & Rate \\
\hline 35 & 30 & $-4.6540 \mathrm{e}-04$ & & $-4.4509 \mathrm{e}-04$ & & $-2.0314 \mathrm{e}-05$ & \\
70 & 60 & $-5.7453 \mathrm{e}-05$ & 3.018 & $-5.6066 \mathrm{e}-05$ & 2.989 & $-1.3871 \mathrm{e}-06$ & 3.872 \\
140 & 120 & $-7.1271 \mathrm{e}-06$ & 3.011 & $-7.0368 \mathrm{e}-06$ & 2.994 & $-9.0276 \mathrm{e}-08$ & 3.942 \\
280 & 240 & $-8.8721 \mathrm{e}-07$ & 3.006 & $-8.8146 \mathrm{e}-07$ & 2.997 & $-5.7520 \mathrm{e}-09$ & 3.972 \\
560 & 480 & $-1.1066 \mathrm{e}-07$ & 3.003 & $-1.1030 \mathrm{e}-07$ & 2.998 & $-3.6290 \mathrm{e}-10$ & 3.986 \\
1120 & 960 & $-1.3818 \mathrm{e}-08$ & 3.002 & $-1.3795 \mathrm{e}-08$ & 2.999 & $-2.2796 \mathrm{e}-11$ & 3.993 \\
\hline \hline Time steps & Cells & $I m\left\{e_{\mathcal{M}}(\tilde{u})\right\}$ & Rate & $\operatorname{Im}\left\{\tilde{e}_{\mathcal{M}}(\tilde{u}, \tilde{\phi})\right\}$ & Rate & $I m\{a c c(\tilde{u}, \tilde{\phi})\}$ & Rate \\
\hline 35 & 30 & $1.1394 \mathrm{e}-03$ & & $9.3597 \mathrm{e}-04$ & & $2.0343 \mathrm{e}-04$ & \\
70 & 60 & $2.9095 \mathrm{e}-04$ & 1.969 & $2.7449 \mathrm{e}-04$ & 1.770 & $1.6466 \mathrm{e}-05$ & 3.627 \\
140 & 120 & $7.3114 \mathrm{e}-05$ & 1.993 & $7.1646 \mathrm{e}-05$ & 1.938 & $1.4684 \mathrm{e}-06$ & 3.487 \\
280 & 240 & $1.8302 \mathrm{e}-05$ & 1.998 & $1.8156 \mathrm{e}-05$ & 1.980 & $1.4582 \mathrm{e}-07$ & 3.332 \\
560 & 480 & $4.5769 \mathrm{e}-06$ & 2.000 & $4.5611 \mathrm{e}-06$ & 1.993 & $1.5845 \mathrm{e}-08$ & 3.202 \\
1120 & 960 & $1.1443 \mathrm{e}-06$ & 2.000 & $1.1425 \mathrm{e}-06$ & 1.997 & $1.8309 \mathrm{e}-09$ & 3.113 \\
\hline
\end{tabular}


TABle 15. Test: IC1 - Q1 - FS2 R2 - AS1 R2

\begin{tabular}{|r|r|rc|rc|rr|}
\hline Time steps & Cells & $\operatorname{Re}\left\{e_{\mathcal{M}}(\tilde{u})\right\}$ & Rate & $\operatorname{Re}\left\{\tilde{e}_{\mathcal{M}}(\tilde{u}, \tilde{\phi})\right\}$ & Rate & $\operatorname{Re}\{a c c(\tilde{u}, \tilde{\phi})\}$ & Rate \\
\hline 35 & 30 & $-4.6540 \mathrm{e}-04$ & & $-4.2382 \mathrm{e}-04$ & & $-4.1579 \mathrm{e}-05$ & \\
70 & 60 & $-5.7453 \mathrm{e}-05$ & 3.018 & $-5.5381 \mathrm{e}-05$ & 2.936 & $-2.0721 \mathrm{e}-06$ & 4.327 \\
140 & 120 & $-7.1271 \mathrm{e}-06$ & 3.011 & $-7.0151 \mathrm{e}-06$ & 2.981 & $-1.1195 \mathrm{e}-07$ & 4.210 \\
280 & 240 & $-8.8721 \mathrm{e}-07$ & 3.006 & $-8.8077 \mathrm{e}-07$ & 2.994 & $-6.4329 \mathrm{e}-09$ & 4.121 \\
560 & 480 & $-1.1066 \mathrm{e}-07$ & 3.003 & $-1.1028 \mathrm{e}-07$ & 2.998 & $-3.8419 \mathrm{e}-10$ & 4.066 \\
1120 & 960 & $-1.3818 \mathrm{e}-08$ & 3.002 & $-1.3794 \mathrm{e}-08$ & 2.999 & $-2.3393 \mathrm{e}-11$ & 4.038 \\
\hline \hline Time steps & Cells & $I m\left\{e_{\mathcal{M}}(\tilde{u})\right\}$ & Rate & $I m\left\{\tilde{e}_{\mathcal{M}}(\tilde{u}, \tilde{\phi})\right\}$ & Rate & $I m\{a c c(\tilde{u}, \tilde{\phi})\}$ & Rate \\
\hline 35 & 30 & $1.1394 \mathrm{e}-03$ & & $1.0869 \mathrm{e}-03$ & & $5.2532 \mathrm{e}-05$ & \\
70 & 60 & $2.9095 \mathrm{e}-04$ & 1.969 & $2.8423 \mathrm{e}-04$ & 1.935 & $6.7242 \mathrm{e}-06$ & 2.966 \\
140 & 120 & $7.3114 \mathrm{e}-05$ & 1.993 & $7.2263 \mathrm{e}-05$ & 1.976 & $8.5172 \mathrm{e}-07$ & 2.981 \\
280 & 240 & $1.8302 \mathrm{e}-05$ & 1.998 & $1.8195 \mathrm{e}-05$ & 1.990 & $1.0707 \mathrm{e}-07$ & 2.992 \\
560 & 480 & $4.5769 \mathrm{e}-06$ & 2.000 & $4.5635 \mathrm{e}-06$ & 1.995 & $1.3417 \mathrm{e}-08$ & 2.996 \\
1120 & 960 & $1.1443 \mathrm{e}-06$ & 2.000 & $1.1426 \mathrm{e}-06$ & 1.998 & $1.6790 \mathrm{e}-09$ & 2.998 \\
\hline
\end{tabular}

TABle 16. Test: IC1 - Q1 - FS2 R1 - AS2 R1

\begin{tabular}{|r|r|rc|rc|rr|}
\hline Time steps & Cells & $\operatorname{Re}\left\{e_{\mathcal{M}}(\tilde{u})\right\}$ & Rate & $\operatorname{Re}\left\{\tilde{e}_{\mathcal{M}}(\tilde{u}, \tilde{\phi})\right\}$ & Rate & $\operatorname{Re}\{a c c(\tilde{u}, \tilde{\phi})\}$ & Rate \\
\hline 35 & 30 & $-4.6629 \mathrm{e}-04$ & & $3.1196 \mathrm{e}-03$ & & $-3.5859 \mathrm{e}-03$ & \\
70 & 60 & $-5.7522 \mathrm{e}-05$ & 3.019 & $3.9342 \mathrm{e}-04$ & 2.987 & $-4.5095 \mathrm{e}-04$ & 2.991 \\
140 & 120 & $-7.1318 \mathrm{e}-06$ & 3.012 & $4.9278 \mathrm{e}-05$ & 2.997 & $-5.6410 \mathrm{e}-05$ & 2.999 \\
280 & 240 & $-8.8752 \mathrm{e}-07$ & 3.006 & $6.1625 \mathrm{e}-06$ & 2.999 & $-7.0500 \mathrm{e}-06$ & 3.000 \\
560 & 480 & $-1.1068 \mathrm{e}-07$ & 3.003 & $7.7038 \mathrm{e}-07$ & 3.000 & $-8.8106 \mathrm{e}-07$ & 3.000 \\
1120 & 960 & $-1.3819 \mathrm{e}-08$ & 3.002 & $9.6298 \mathrm{e}-08$ & 3.000 & $-1.1012 \mathrm{e}-07$ & 3.000 \\
\hline \hline Time steps & Cells & $I m\left\{e_{\mathcal{M}}(\tilde{u})\right\}$ & Rate & $\operatorname{Im}\left\{\tilde{e}_{\mathcal{M}}(\tilde{u}, \tilde{\phi})\right\}$ & Rate & $\operatorname{Im}\{a c c(\tilde{u}, \tilde{\phi})\}$ & Rate \\
\hline 35 & 30 & $9.4943 \mathrm{e}-04$ & & $6.7391 \mathrm{e}-04$ & & $2.7551 \mathrm{e}-04$ & \\
70 & 60 & $2.6707 \mathrm{e}-04$ & 1.830 & $2.4950 \mathrm{e}-04$ & 1.434 & $1.7570 \mathrm{e}-05$ & 3.971 \\
140 & 120 & $7.0125 \mathrm{e}-05$ & 1.929 & $6.9018 \mathrm{e}-05$ & 1.854 & $1.1072 \mathrm{e}-06$ & 3.988 \\
280 & 240 & $1.7928 \mathrm{e}-05$ & 1.968 & $1.7859 \mathrm{e}-05$ & 1.950 & $6.9455 \mathrm{e}-08$ & 3.995 \\
560 & 480 & $4.5302 \mathrm{e}-06$ & 1.985 & $4.5259 \mathrm{e}-06$ & 1.980 & $4.3484 \mathrm{e}-09$ & 3.998 \\
1120 & 960 & $1.1385 \mathrm{e}-06$ & 1.992 & $1.1382 \mathrm{e}-06$ & 1.991 & $2.7200 \mathrm{e}-10$ & 3.999 \\
\hline
\end{tabular}


TABle 17. Test: IC1 - Q1 - FS2 R1 - AS2 R2

\begin{tabular}{|r|r|rc|rc|rc|}
\hline Time steps & Cells & $\operatorname{Re}\left\{e_{\mathcal{M}}(\tilde{u})\right\}$ & Rate & $\operatorname{Re}\left\{\tilde{e}_{\mathcal{M}}(\tilde{u}, \tilde{\phi})\right\}$ & Rate & $\operatorname{Re}\{a c c(\tilde{u}, \tilde{\phi})\}$ & Rate \\
\hline 35 & 30 & $-4.6629 \mathrm{e}-04$ & & $-4.1128 \mathrm{e}-04$ & & $-5.5011 \mathrm{e}-05$ & \\
70 & 60 & $-5.7522 \mathrm{e}-05$ & 3.019 & $-5.5821 \mathrm{e}-05$ & 2.881 & $-1.7015 \mathrm{e}-06$ & 5.015 \\
140 & 120 & $-7.1318 \mathrm{e}-06$ & 3.012 & $-7.0811 \mathrm{e}-06$ & 2.979 & $-5.0735 \mathrm{e}-08$ & 5.068 \\
280 & 240 & $-8.8752 \mathrm{e}-07$ & 3.006 & $-8.8610 \mathrm{e}-07$ & 2.998 & $-1.4205 \mathrm{e}-09$ & 5.158 \\
560 & 480 & $-1.1068 \mathrm{e}-07$ & 3.003 & $-1.1065 \mathrm{e}-07$ & 3.001 & $-3.3975 \mathrm{e}-11$ & 5.386 \\
1120 & 960 & $-1.3819 \mathrm{e}-08$ & 3.002 & $-1.3819 \mathrm{e}-08$ & 3.001 & $-4.2334 \mathrm{e}-13$ & 6.327 \\
\hline \hline Time steps & Cells & $I m\left\{e_{\mathcal{M}}(\tilde{u})\right\}$ & Rate & $I m\left\{\tilde{e}_{\mathcal{M}}(\tilde{u}, \tilde{\phi})\right\}$ & Rate & $I m\{a c c(\tilde{u}, \tilde{\phi})\}$ & Rate \\
\hline 35 & 30 & $9.4943 \mathrm{e}-04$ & & $1.2861 \mathrm{e}-03$ & & $-3.3668 \mathrm{e}-04$ & \\
70 & 60 & $2.6707 \mathrm{e}-04$ & 1.830 & $2.8847 \mathrm{e}-04$ & 2.156 & $-2.1404 \mathrm{e}-05$ & 3.975 \\
140 & 120 & $7.0125 \mathrm{e}-05$ & 1.929 & $7.1468 \mathrm{e}-05$ & 2.013 & $-1.3431 \mathrm{e}-06$ & 3.994 \\
280 & 240 & $1.7928 \mathrm{e}-05$ & 1.968 & $1.8012 \mathrm{e}-05$ & 1.988 & $-8.4022 \mathrm{e}-08$ & 3.999 \\
560 & 480 & $4.5302 \mathrm{e}-06$ & 1.985 & $4.5355 \mathrm{e}-06$ & 1.990 & $-5.2524 \mathrm{e}-09$ & 4.000 \\
1120 & 960 & $1.1385 \mathrm{e}-06$ & 1.992 & $1.1388 \mathrm{e}-06$ & 1.994 & $-3.2828 \mathrm{e}-10$ & 4.000 \\
\hline
\end{tabular}

TABle 18. Test: IC1 - Q1 - FS2 R2 - AS2 R1

\begin{tabular}{|r|r|rc|rc|rr|}
\hline Time steps & Cells & $\operatorname{Re}\left\{e_{\mathcal{M}}(\tilde{u})\right\}$ & Rate & $\operatorname{Re}\left\{\tilde{e}_{\mathcal{M}}(\tilde{u}, \tilde{\phi})\right\}$ & Rate & $\operatorname{Re}\{a c c(\tilde{u}, \tilde{\phi})\}$ & Rate \\
\hline 35 & 30 & $-4.6540 \mathrm{e}-04$ & & $-4.7075 \mathrm{e}-04$ & & $5.3451 \mathrm{e}-06$ & \\
70 & 60 & $-5.7453 \mathrm{e}-05$ & 3.018 & $-5.7677 \mathrm{e}-05$ & 3.029 & $2.2426 \mathrm{e}-07$ & 4.575 \\
140 & 120 & $-7.1271 \mathrm{e}-06$ & 3.011 & $-7.1370 \mathrm{e}-06$ & 3.015 & $9.8985 \mathrm{e}-09$ & 4.502 \\
280 & 240 & $-8.8721 \mathrm{e}-07$ & 3.006 & $-8.8769 \mathrm{e}-07$ & 3.007 & $4.8007 \mathrm{e}-10$ & 4.366 \\
560 & 480 & $-1.1066 \mathrm{e}-07$ & 3.003 & $-1.1069 \mathrm{e}-07$ & 3.004 & $2.5519 \mathrm{e}-11$ & 4.234 \\
1120 & 960 & $-1.3818 \mathrm{e}-08$ & 3.002 & $-1.3819 \mathrm{e}-08$ & 3.002 & $1.4415 \mathrm{e}-12$ & 4.146 \\
\hline \hline Time steps & Cells & $I m\left\{e_{\mathcal{M}}(\tilde{u})\right\}$ & Rate & $I m\left\{\tilde{e}_{\mathcal{M}}(\tilde{u}, \tilde{\phi})\right\}$ & Rate & $I m\{a c c(\tilde{u}, \tilde{\phi})\}$ & Rate \\
\hline 35 & 30 & $1.1394 \mathrm{e}-03$ & & $9.7840 \mathrm{e}-04$ & & $1.6100 \mathrm{e}-04$ & \\
70 & 60 & $2.9095 \mathrm{e}-04$ & 1.969 & $2.8092 \mathrm{e}-04$ & 1.800 & $1.0035 \mathrm{e}-05$ & 4.004 \\
140 & 120 & $7.3114 \mathrm{e}-05$ & 1.993 & $7.2489 \mathrm{e}-05$ & 1.954 & $6.2541 \mathrm{e}-07$ & 4.004 \\
280 & 240 & $1.8302 \mathrm{e}-05$ & 1.998 & $1.8263 \mathrm{e}-05$ & 1.989 & $3.9020 \mathrm{e}-08$ & 4.003 \\
560 & 480 & $4.5769 \mathrm{e}-06$ & 2.000 & $4.5745 \mathrm{e}-06$ & 1.997 & $2.4365 \mathrm{e}-09$ & 4.001 \\
1120 & 960 & $1.1443 \mathrm{e}-06$ & 2.000 & $1.1442 \mathrm{e}-06$ & 1.999 & $1.5220 \mathrm{e}-10$ & 4.001 \\
\hline
\end{tabular}


TABle 19. Test: IC1 - Q1 - FS2 R2 - AS2 R2

\begin{tabular}{|r|r|rc|rc|rc|}
\hline Time steps & Cells & $\operatorname{Re}\left\{e_{\mathcal{M}}(\tilde{u})\right\}$ & Rate & $\operatorname{Re}\left\{\tilde{e}_{\mathcal{M}}(\tilde{u}, \tilde{\phi})\right\}$ & Rate & $\operatorname{Re}\{a c c(\tilde{u}, \tilde{\phi})\}$ & Rate \\
\hline 35 & 30 & $-4.6540 \mathrm{e}-04$ & & $-4.4903 \mathrm{e}-04$ & & $-1.6376 \mathrm{e}-05$ & \\
70 & 60 & $-5.7453 \mathrm{e}-05$ & 3.018 & $-5.6985 \mathrm{e}-05$ & 2.978 & $-4.6813 \mathrm{e}-07$ & 5.129 \\
140 & 120 & $-7.1271 \mathrm{e}-06$ & 3.011 & $-7.1152 \mathrm{e}-06$ & 3.002 & $-1.1891 \mathrm{e}-08$ & 5.299 \\
280 & 240 & $-8.8721 \mathrm{e}-07$ & 3.006 & $-8.8700 \mathrm{e}-07$ & 3.004 & $-2.0278 \mathrm{e}-10$ & 5.874 \\
560 & 480 & $-1.1066 \mathrm{e}-07$ & 3.003 & $-1.1067 \mathrm{e}-07$ & 3.003 & $4.1911 \mathrm{e}-12$ & 5.596 \\
1120 & 960 & $-1.3818 \mathrm{e}-08$ & 3.002 & $-1.3819 \mathrm{e}-08$ & 3.002 & $8.4486 \mathrm{e}-13$ & 2.311 \\
\hline \hline Time steps & Cells & $I m\left\{e_{\mathcal{M}}(\tilde{u})\right\}$ & Rate & $\operatorname{Im}\left\{\tilde{e}_{\mathcal{M}}(\tilde{u}, \tilde{\phi})\right\}$ & Rate & $\operatorname{Im}\{a c c(\tilde{u}, \tilde{\phi})\}$ & Rate \\
\hline 35 & 30 & $1.1394 \mathrm{e}-03$ & & $1.1366 \mathrm{e}-03$ & & $2.8316 \mathrm{e}-06$ & \\
70 & 60 & $2.9095 \mathrm{e}-04$ & 1.969 & $2.9089 \mathrm{e}-04$ & 1.966 & $6.1749 \mathrm{e}-08$ & 5.519 \\
140 & 120 & $7.3114 \mathrm{e}-05$ & 1.993 & $7.3113 \mathrm{e}-05$ & 1.992 & $1.4886 \mathrm{e}-09$ & 5.374 \\
280 & 240 & $1.8302 \mathrm{e}-05$ & 1.998 & $1.8302 \mathrm{e}-05$ & 1.998 & $3.9477 \mathrm{e}-11$ & 5.237 \\
560 & 480 & $4.5769 \mathrm{e}-06$ & 2.000 & $4.5769 \mathrm{e}-06$ & 2.000 & $1.1228 \mathrm{e}-12$ & 5.136 \\
1120 & 960 & $1.1443 \mathrm{e}-06$ & 2.000 & $1.1443 \mathrm{e}-06$ & 2.000 & $3.3502 \mathrm{e}-14$ & 5.067 \\
\hline
\end{tabular}

4.1.3. Smooth initial condition with TVD method. The results in Tables 20 - 27 correspond to tests using the high-fidelity TVD method for the forward problem. This is a nonlinear method and loses accuracy at solution extrema. The converence rate of the cell averages may be considered as $s_{u}=4 / 3$, which has been verified computationally. The results show that for the quantity of interest Q1, the convergence rate of the error $e_{\mathcal{M}}(\tilde{u})$ is always 2 , in contrast to the results using second order upwind for the forward problem which were sometimes as high as 3 . An interesting byproduct is that the computable error estimates using only linear reconstruction both in Table 20 and Table 24 are more accurate relative to the size of the true error than when the second order upwind method was used for the forward solution.

Again, the results in these two tables are not as pessimistic as predicted by Theorem 3.1. This is possibly related to the particular choices of initial condition for the forward problem and the quantity of interest. Other choices are explored later. The results, otherwise, are as observed in Section 4.1.2 in that when the adjoint method is first order there is not a significant difference in the results using a linear reconstruction for one of the forward or adjoint solution data versus quadratic for the other, or using quadratic for both, as long as they are not both chosen to be linear. However, when the adjoint method is second order, the best result comes from using quadratic reconstruction for both the forward and adjoint data. 
TABLE 20. Test: IC1 - Q1 - FS3 R1 - AS1 R1

\begin{tabular}{|r|r|rc|rr|rr|}
\hline Time steps & Cells & $\operatorname{Re}\left\{e_{\mathcal{M}}(\tilde{u})\right\}$ & Rate & $\operatorname{Re}\left\{\tilde{e}_{\mathcal{M}}(\tilde{u}, \tilde{\phi})\right\}$ & Rate & $\operatorname{Re}\{a c c(\tilde{u}, \tilde{\phi})\}$ & Rate \\
\hline 35 & 30 & $-3.8256 \mathrm{e}-03$ & & $-2.4090 \mathrm{e}-04$ & & $-3.5847 \mathrm{e}-03$ & \\
70 & 60 & $-8.6746 \mathrm{e}-04$ & 2.141 & $-4.0721 \mathrm{e}-04$ & -0.757 & $-4.6025 \mathrm{e}-04$ & 2.961 \\
140 & 120 & $-2.0588 \mathrm{e}-04$ & 2.075 & $-1.4773 \mathrm{e}-04$ & 1.463 & $-5.8146 \mathrm{e}-05$ & 2.985 \\
280 & 240 & $-5.0152 \mathrm{e}-05$ & 2.037 & $-4.2849 \mathrm{e}-05$ & 1.786 & $-7.3024 \mathrm{e}-06$ & 2.993 \\
560 & 480 & $-1.2382 \mathrm{e}-05$ & 2.018 & $-1.1467 \mathrm{e}-05$ & 1.902 & $-9.1481 \mathrm{e}-07$ & 2.997 \\
1120 & 960 & $-3.0769 \mathrm{e}-06$ & 2.009 & $-2.9624 \mathrm{e}-06$ & 1.953 & $-1.1447 \mathrm{e}-07$ & 2.998 \\
\hline \hline Time steps & Cells & $I m\left\{e_{\mathcal{M}}(\tilde{u})\right\}$ & Rate & $\operatorname{Im}\left\{\tilde{e}_{\mathcal{M}}(\tilde{u}, \tilde{\phi})\right\}$ & Rate & $\operatorname{Im}\{a c c(\tilde{u}, \tilde{\phi})\}$ & Rate \\
\hline 35 & 30 & $6.7291 \mathrm{e}-04$ & & $3.8310 \mathrm{e}-04$ & & $2.8981 \mathrm{e}-04$ & \\
70 & 60 & $2.3527 \mathrm{e}-04$ & 1.516 & $2.1329 \mathrm{e}-04$ & 0.845 & $2.1983 \mathrm{e}-05$ & 3.721 \\
140 & 120 & $6.6322 \mathrm{e}-05$ & 1.827 & $6.4506 \mathrm{e}-05$ & 1.725 & $1.8162 \mathrm{e}-06$ & 3.597 \\
280 & 240 & $1.7463 \mathrm{e}-05$ & 1.925 & $1.7295 \mathrm{e}-05$ & 1.899 & $1.6764 \mathrm{e}-07$ & 3.437 \\
560 & 480 & $4.4725 \mathrm{e}-06$ & 1.965 & $4.4553 \mathrm{e}-06$ & 1.957 & $1.7211 \mathrm{e}-08$ & 3.284 \\
1120 & 960 & $1.1313 \mathrm{e}-06$ & 1.983 & $1.1294 \mathrm{e}-06$ & 1.980 & $1.9163 \mathrm{e}-09$ & 3.167 \\
\hline
\end{tabular}

TABle 21. Test: IC1 - Q1 - FS3 R1 - AS1 R2

\begin{tabular}{|r|r|rc|rc|rr|}
\hline Time steps & Cells & $\operatorname{Re}\left\{e_{\mathcal{M}}(\tilde{u})\right\}$ & Rate & $\operatorname{Re}\left\{\tilde{e}_{\mathcal{M}}(\tilde{u}, \tilde{\phi})\right\}$ & Rate & $\operatorname{Re}\{a c c(\tilde{u}, \tilde{\phi})\}$ & Rate \\
\hline 35 & 30 & $-3.8256 \mathrm{e}-03$ & & $-3.5982 \mathrm{e}-03$ & & $-2.2732 \mathrm{e}-04$ & \\
70 & 60 & $-8.6746 \mathrm{e}-04$ & 2.141 & $-8.4567 \mathrm{e}-04$ & 2.089 & $-2.1788 \mathrm{e}-05$ & 3.383 \\
140 & 120 & $-2.0588 \mathrm{e}-04$ & 2.075 & $-2.0342 \mathrm{e}-04$ & 2.056 & $-2.4559 \mathrm{e}-06$ & 3.149 \\
280 & 240 & $-5.0152 \mathrm{e}-05$ & 2.037 & $-4.9856 \mathrm{e}-05$ & 2.029 & $-2.9538 \mathrm{e}-07$ & 3.056 \\
560 & 480 & $-1.2382 \mathrm{e}-05$ & 2.018 & $-1.2345 \mathrm{e}-05$ & 2.014 & $-3.6369 \mathrm{e}-08$ & 3.022 \\
1120 & 960 & $-3.0769 \mathrm{e}-06$ & 2.009 & $-3.0723 \mathrm{e}-06$ & 2.007 & $-4.5176 \mathrm{e}-09$ & 3.009 \\
\hline \hline Time steps & Cells & $I m\left\{e_{\mathcal{M}}(\tilde{u})\right\}$ & Rate & $\operatorname{Im}\left\{\tilde{e}_{\mathcal{M}}(\tilde{u}, \tilde{\phi})\right\}$ & Rate & $I m\{a c c(\tilde{u}, \tilde{\phi})\}$ & Rate \\
\hline 35 & 30 & $6.7291 \mathrm{e}-04$ & & $9.7938 \mathrm{e}-04$ & & $-3.0647 \mathrm{e}-04$ & \\
70 & 60 & $2.3527 \mathrm{e}-04$ & 1.516 & $2.5177 \mathrm{e}-04$ & 1.960 & $-1.6494 \mathrm{e}-05$ & 4.216 \\
140 & 120 & $6.6322 \mathrm{e}-05$ & 1.827 & $6.6941 \mathrm{e}-05$ & 1.911 & $-6.1870 \mathrm{e}-07$ & 4.737 \\
280 & 240 & $1.7463 \mathrm{e}-05$ & 1.925 & $1.7448 \mathrm{e}-05$ & 1.940 & $1.4644 \mathrm{e}-08$ & 5.401 \\
560 & 480 & $4.4725 \mathrm{e}-06$ & 1.965 & $4.4649 \mathrm{e}-06$ & 1.966 & $7.6248 \mathrm{e}-09$ & 0.942 \\
1120 & 960 & $1.1313 \mathrm{e}-06$ & 1.983 & $1.1300 \mathrm{e}-06$ & 1.982 & $1.3165 \mathrm{e}-09$ & 2.534 \\
\hline
\end{tabular}


TABLE 22. Test: IC1 - Q1 - FS3 R2 - AS1 R1

\begin{tabular}{|r|r|rc|rc|rc|}
\hline Time steps & Cells & $\operatorname{Re}\left\{e_{\mathcal{M}}(\tilde{u})\right\}$ & Rate & $\operatorname{Re}\left\{\tilde{e}_{\mathcal{M}}(\tilde{u}, \tilde{\phi})\right\}$ & Rate & $\operatorname{Re}\{a c c(\tilde{u}, \tilde{\phi})\}$ & Rate \\
\hline 35 & 30 & $-3.8246 \mathrm{e}-03$ & & $-3.6552 \mathrm{e}-03$ & & $-1.6934 \mathrm{e}-04$ & \\
70 & 60 & $-8.6739 \mathrm{e}-04$ & 2.141 & $-8.4747 \mathrm{e}-04$ & 2.109 & $-1.9919 \mathrm{e}-05$ & 3.088 \\
140 & 120 & $-2.0587 \mathrm{e}-04$ & 2.075 & $-2.0347 \mathrm{e}-04$ & 2.058 & $-2.3968 \mathrm{e}-06$ & 3.055 \\
280 & 240 & $-5.0152 \mathrm{e}-05$ & 2.037 & $-4.9858 \mathrm{e}-05$ & 2.029 & $-2.9352 \mathrm{e}-07$ & 3.030 \\
560 & 480 & $-1.2382 \mathrm{e}-05$ & 2.018 & $-1.2345 \mathrm{e}-05$ & 2.014 & $-3.6311 \mathrm{e}-08$ & 3.015 \\
1120 & 960 & $-3.0769 \mathrm{e}-06$ & 2.009 & $-3.0723 \mathrm{e}-06$ & 2.007 & $-4.5158 \mathrm{e}-09$ & 3.007 \\
\hline \hline Time steps & Cells & $I m\left\{e_{\mathcal{M}}(\tilde{u})\right\}$ & Rate & $I m\left\{\tilde{e}_{\mathcal{M}}(\tilde{u}, \tilde{\phi})\right\}$ & Rate & $I m\{a c c(\tilde{u}, \tilde{\phi})\}$ & Rate \\
\hline 35 & 30 & $8.6160 \mathrm{e}-04$ & & $6.7674 \mathrm{e}-04$ & & $1.8486 \mathrm{e}-04$ & \\
70 & 60 & $2.5912 \mathrm{e}-04$ & 1.733 & $2.4380 \mathrm{e}-04$ & 1.473 & $1.5322 \mathrm{e}-05$ & 3.593 \\
140 & 120 & $6.9310 \mathrm{e}-05$ & 1.902 & $6.7913 \mathrm{e}-05$ & 1.844 & $1.3970 \mathrm{e}-06$ & 3.455 \\
280 & 240 & $1.7836 \mathrm{e}-05$ & 1.958 & $1.7695 \mathrm{e}-05$ & 1.940 & $1.4136 \mathrm{e}-07$ & 3.305 \\
560 & 480 & $4.5193 \mathrm{e}-06$ & 1.981 & $4.5037 \mathrm{e}-06$ & 1.974 & $1.5566 \mathrm{e}-08$ & 3.183 \\
1120 & 960 & $1.1371 \mathrm{e}-06$ & 1.991 & $1.1353 \mathrm{e}-06$ & 1.988 & $1.8134 \mathrm{e}-09$ & 3.102 \\
\hline
\end{tabular}

TABle 23. Test: IC1 - Q1 - FS3 R2 - AS1 R2

\begin{tabular}{|r|r|rc|rc|rr|}
\hline Time steps & Cells & $\operatorname{Re}\left\{e_{\mathcal{M}}(\tilde{u})\right\}$ & Rate & $\operatorname{Re}\left\{\tilde{e}_{\mathcal{M}}(\tilde{u}, \tilde{\phi})\right\}$ & Rate & $\operatorname{Re}\{a c c(\tilde{u}, \tilde{\phi})\}$ & Rate \\
\hline 35 & 30 & $-3.8246 \mathrm{e}-03$ & & $-3.6341 \mathrm{e}-03$ & & $-1.9047 \mathrm{e}-04$ & \\
70 & 60 & $-8.6739 \mathrm{e}-04$ & 2.141 & $-8.4679 \mathrm{e}-04$ & 2.102 & $-2.0602 \mathrm{e}-05$ & 3.209 \\
140 & 120 & $-2.0587 \mathrm{e}-04$ & 2.075 & $-2.0345 \mathrm{e}-04$ & 2.057 & $-2.4184 \mathrm{e}-06$ & 3.091 \\
280 & 240 & $-5.0152 \mathrm{e}-05$ & 2.037 & $-4.9857 \mathrm{e}-05$ & 2.029 & $-2.9420 \mathrm{e}-07$ & 3.039 \\
560 & 480 & $-1.2382 \mathrm{e}-05$ & 2.018 & $-1.2345 \mathrm{e}-05$ & 2.014 & $-3.6332 \mathrm{e}-08$ & 3.017 \\
1120 & 960 & $-3.0769 \mathrm{e}-06$ & 2.009 & $-3.0723 \mathrm{e}-06$ & 2.007 & $-4.5164 \mathrm{e}-09$ & 3.008 \\
\hline \hline Time steps & Cells & $I m\left\{e_{\mathcal{M}}(\tilde{u})\right\}$ & Rate & $\operatorname{Im}\left\{\tilde{e}_{\mathcal{M}}(\tilde{u}, \tilde{\phi})\right\}$ & Rate & $I m\{a c c(\tilde{u}, \tilde{\phi})\}$ & Rate \\
\hline 35 & 30 & $8.6160 \mathrm{e}-04$ & & $8.2834 \mathrm{e}-04$ & & $3.3258 \mathrm{e}-05$ & \\
70 & 60 & $2.5912 \mathrm{e}-04$ & 1.733 & $2.5357 \mathrm{e}-04$ & 1.708 & $5.5501 \mathrm{e}-06$ & 2.583 \\
140 & 120 & $6.9310 \mathrm{e}-05$ & 1.902 & $6.8531 \mathrm{e}-05$ & 1.888 & $7.7933 \mathrm{e}-07$ & 2.832 \\
280 & 240 & $1.7836 \mathrm{e}-05$ & 1.958 & $1.7734 \mathrm{e}-05$ & 1.950 & $1.0257 \mathrm{e}-07$ & 2.926 \\
560 & 480 & $4.5193 \mathrm{e}-06$ & 1.981 & $4.5061 \mathrm{e}-06$ & 1.977 & $1.3136 \mathrm{e}-08$ & 2.965 \\
1120 & 960 & $1.1371 \mathrm{e}-06$ & 1.991 & $1.1355 \mathrm{e}-06$ & 1.989 & $1.6615 \mathrm{e}-09$ & 2.983 \\
\hline
\end{tabular}


TABLE 24. Test: IC1 - Q1 - FS3 R1 - AS2 R1

\begin{tabular}{|r|r|rc|rr|rr|}
\hline Time steps & Cells & $\operatorname{Re}\left\{e_{\mathcal{M}}(\tilde{u})\right\}$ & Rate & $\operatorname{Re}\left\{\tilde{e}_{\mathcal{M}}(\tilde{u}, \tilde{\phi})\right\}$ & Rate & $\operatorname{Re}\{a c c(\tilde{u}, \tilde{\phi})\}$ & Rate \\
\hline 35 & 30 & $-3.8256 \mathrm{e}-03$ & & $-2.4955 \mathrm{e}-04$ & & $-3.5760 \mathrm{e}-03$ & \\
70 & 60 & $-8.6746 \mathrm{e}-04$ & 2.141 & $-4.1682 \mathrm{e}-04$ & -0.740 & $-4.5064 \mathrm{e}-04$ & 2.988 \\
140 & 120 & $-2.0588 \mathrm{e}-04$ & 2.075 & $-1.4947 \mathrm{e}-04$ & 1.480 & $-5.6400 \mathrm{e}-05$ & 2.998 \\
280 & 240 & $-5.0152 \mathrm{e}-05$ & 2.037 & $-4.3102 \mathrm{e}-05$ & 1.794 & $-7.0497 \mathrm{e}-06$ & 3.000 \\
560 & 480 & $-1.2382 \mathrm{e}-05$ & 2.018 & $-1.1501 \mathrm{e}-05$ & 1.906 & $-8.8106 \mathrm{e}-07$ & 3.000 \\
1120 & 960 & $-3.0769 \mathrm{e}-06$ & 2.009 & $-2.9667 \mathrm{e}-06$ & 1.955 & $-1.1012 \mathrm{e}-07$ & 3.000 \\
\hline \hline Time steps & Cells & $I m\left\{e_{\mathcal{M}}(\tilde{u})\right\}$ & Rate & $I m\left\{\tilde{e}_{\mathcal{M}}(\tilde{u}, \tilde{\phi})\right\}$ & Rate & $\operatorname{Im}\{a c c(\tilde{u}, \tilde{\phi})\}$ & Rate \\
\hline 35 & 30 & $6.7291 \mathrm{e}-04$ & & $3.9247 \mathrm{e}-04$ & & $2.8043 \mathrm{e}-04$ & \\
70 & 60 & $2.3527 \mathrm{e}-04$ & 1.516 & $2.1743 \mathrm{e}-04$ & 0.852 & $1.7840 \mathrm{e}-05$ & 3.975 \\
140 & 120 & $6.6322 \mathrm{e}-05$ & 1.827 & $6.5199 \mathrm{e}-05$ & 1.738 & $1.1228 \mathrm{e}-06$ & 3.990 \\
280 & 240 & $1.7463 \mathrm{e}-05$ & 1.925 & $1.7392 \mathrm{e}-05$ & 1.906 & $7.0391 \mathrm{e}-08$ & 3.996 \\
560 & 480 & $4.4725 \mathrm{e}-06$ & 1.965 & $4.4681 \mathrm{e}-06$ & 1.961 & $4.4057 \mathrm{e}-09$ & 3.998 \\
1120 & 960 & $1.1313 \mathrm{e}-06$ & 1.983 & $1.1310 \mathrm{e}-06$ & 1.982 & $2.7554 \mathrm{e}-10$ & 3.999 \\
\hline
\end{tabular}

TABle 25. Test: IC1 - Q1 - FS3 R1 - AS2 R2

\begin{tabular}{|r|r|rc|rc|rr|}
\hline Time steps & Cells & $\operatorname{Re}\left\{e_{\mathcal{M}}(\tilde{u})\right\}$ & Rate & $\operatorname{Re}\left\{\tilde{e}_{\mathcal{M}}(\tilde{u}, \tilde{\phi})\right\}$ & Rate & $\operatorname{Re}\{a c c(\tilde{u}, \tilde{\phi})\}$ & Rate \\
\hline 35 & 30 & $-3.8256 \mathrm{e}-03$ & & $-3.7687 \mathrm{e}-03$ & & $-5.6836 \mathrm{e}-05$ & \\
70 & 60 & $-8.6746 \mathrm{e}-04$ & 2.141 & $-8.6570 \mathrm{e}-04$ & 2.122 & $-1.7573 \mathrm{e}-06$ & 5.015 \\
140 & 120 & $-2.0588 \mathrm{e}-04$ & 2.075 & $-2.0582 \mathrm{e}-04$ & 2.072 & $-5.2437 \mathrm{e}-08$ & 5.067 \\
280 & 240 & $-5.0152 \mathrm{e}-05$ & 2.037 & $-5.0150 \mathrm{e}-05$ & 2.037 & $-1.4729 \mathrm{e}-09$ & 5.154 \\
560 & 480 & $-1.2382 \mathrm{e}-05$ & 2.018 & $-1.2382 \mathrm{e}-05$ & 2.018 & $-3.5601 \mathrm{e}-11$ & 5.371 \\
1120 & 960 & $-3.0769 \mathrm{e}-06$ & 2.009 & $-3.0769 \mathrm{e}-06$ & 2.009 & $-4.7301 \mathrm{e}-13$ & 6.234 \\
\hline \hline Time steps & Cells & $I m\left\{e_{\mathcal{M}}(\tilde{u})\right\}$ & Rate & $\operatorname{Im}\left\{\tilde{e}_{\mathcal{M}}(\tilde{u}, \tilde{\phi})\right\}$ & Rate & $I m\{a c c(\tilde{u}, \tilde{\phi})\}$ & Rate \\
\hline 35 & 30 & $6.7291 \mathrm{e}-04$ & & $1.0048 \mathrm{e}-03$ & & $-3.3194 \mathrm{e}-04$ & \\
70 & 60 & $2.3527 \mathrm{e}-04$ & 1.516 & $2.5643 \mathrm{e}-04$ & 1.970 & $-2.1155 \mathrm{e}-05$ & 3.972 \\
140 & 120 & $6.6322 \mathrm{e}-05$ & 1.827 & $6.7651 \mathrm{e}-05$ & 1.922 & $-1.3284 \mathrm{e}-06$ & 3.993 \\
280 & 240 & $1.7463 \mathrm{e}-05$ & 1.925 & $1.7546 \mathrm{e}-05$ & 1.947 & $-8.3118 \mathrm{e}-08$ & 3.998 \\
560 & 480 & $4.4725 \mathrm{e}-06$ & 1.965 & $4.4777 \mathrm{e}-06$ & 1.970 & $-5.1962 \mathrm{e}-09$ & 4.000 \\
1120 & 960 & $1.1313 \mathrm{e}-06$ & 1.983 & $1.1316 \mathrm{e}-06$ & 1.984 & $-3.2478 \mathrm{e}-10$ & 4.000 \\
\hline
\end{tabular}


TABLE 26. Test: IC1 - Q1 - FS3 R2 - AS2 R1

\begin{tabular}{|r|r|rc|rc|rr|}
\hline Time steps & Cells & $\operatorname{Re}\left\{e_{\mathcal{M}}(\tilde{u})\right\}$ & Rate & $\operatorname{Re}\left\{\tilde{e}_{\mathcal{M}}(\tilde{u}, \tilde{\phi})\right\}$ & Rate & $\operatorname{Re}\{a c c(\tilde{u}, \tilde{\phi})\}$ & Rate \\
\hline 35 & 30 & $-3.8246 \mathrm{e}-03$ & & $-3.8279 \mathrm{e}-03$ & & $3.3371 \mathrm{e}-06$ & \\
70 & 60 & $-8.6739 \mathrm{e}-04$ & 2.141 & $-8.6756 \mathrm{e}-04$ & 2.142 & $1.6623 \mathrm{e}-07$ & 4.327 \\
140 & 120 & $-2.0587 \mathrm{e}-04$ & 2.075 & $-2.0588 \mathrm{e}-04$ & 2.075 & $8.1668 \mathrm{e}-09$ & 4.347 \\
280 & 240 & $-5.0152 \mathrm{e}-05$ & 2.037 & $-5.0152 \mathrm{e}-05$ & 2.037 & $4.2723 \mathrm{e}-10$ & 4.257 \\
560 & 480 & $-1.2382 \mathrm{e}-05$ & 2.018 & $-1.2382 \mathrm{e}-05$ & 2.018 & $2.3887 \mathrm{e}-11$ & 4.161 \\
1120 & 960 & $-3.0769 \mathrm{e}-06$ & 2.009 & $-3.0769 \mathrm{e}-06$ & 2.009 & $1.3914 \mathrm{e}-12$ & 4.102 \\
\hline \hline Time steps & Cells & $I m\left\{e_{\mathcal{M}}(\tilde{u})\right\}$ & Rate & $I m\left\{\tilde{e}_{\mathcal{M}}(\tilde{u}, \tilde{\phi})\right\}$ & Rate & $I m\{a c c(\tilde{u}, \tilde{\phi})\}$ & Rate \\
\hline 35 & 30 & $8.6160 \mathrm{e}-04$ & & $6.9625 \mathrm{e}-04$ & & $1.6535 \mathrm{e}-04$ & \\
70 & 60 & $2.5912 \mathrm{e}-04$ & 1.733 & $2.4882 \mathrm{e}-04$ & 1.484 & $1.0297 \mathrm{e}-05$ & 4.005 \\
140 & 120 & $6.9310 \mathrm{e}-05$ & 1.902 & $6.8670 \mathrm{e}-05$ & 1.857 & $6.4092 \mathrm{e}-07$ & 4.006 \\
280 & 240 & $1.7836 \mathrm{e}-05$ & 1.958 & $1.7796 \mathrm{e}-05$ & 1.948 & $3.9955 \mathrm{e}-08$ & 4.004 \\
560 & 480 & $4.5193 \mathrm{e}-06$ & 1.981 & $4.5168 \mathrm{e}-06$ & 1.978 & $2.4937 \mathrm{e}-09$ & 4.002 \\
1120 & 960 & $1.1371 \mathrm{e}-06$ & 1.991 & $1.1370 \mathrm{e}-06$ & 1.990 & $1.5574 \mathrm{e}-10$ & 4.001 \\
\hline
\end{tabular}

TABLE 27. Test: IC1 - Q1 - FS3 R2 - AS2 R2

\begin{tabular}{|r|r|rc|rc|rr|}
\hline Time steps & Cells & $\operatorname{Re}\left\{e_{\mathcal{M}}(\tilde{u})\right\}$ & Rate & $\operatorname{Re}\left\{\tilde{e}_{\mathcal{M}}(\tilde{u}, \tilde{\phi})\right\}$ & Rate & $\operatorname{Re}\{a c c(\tilde{u}, \tilde{\phi})\}$ & Rate \\
\hline 35 & 30 & $-3.8246 \mathrm{e}-03$ & & $-3.8063 \mathrm{e}-03$ & & $-1.8240 \mathrm{e}-05$ & \\
70 & 60 & $-8.6739 \mathrm{e}-04$ & 2.141 & $-8.6687 \mathrm{e}-04$ & 2.135 & $-5.2426 \mathrm{e}-07$ & 5.121 \\
140 & 120 & $-2.0587 \mathrm{e}-04$ & 2.075 & $-2.0586 \mathrm{e}-04$ & 2.074 & $-1.3596 \mathrm{e}-08$ & 5.269 \\
280 & 240 & $-5.0152 \mathrm{e}-05$ & 2.037 & $-5.0151 \mathrm{e}-05$ & 2.037 & $-2.5522 \mathrm{e}-10$ & 5.735 \\
560 & 480 & $-1.2382 \mathrm{e}-05$ & 2.018 & $-1.2382 \mathrm{e}-05$ & 2.018 & $2.5653 \mathrm{e}-12$ & 6.636 \\
1120 & 960 & $-3.0769 \mathrm{e}-06$ & 2.009 & $-3.0769 \mathrm{e}-06$ & 2.009 & $7.9479 \mathrm{e}-13$ & 1.690 \\
\hline \hline Time steps & Cells & $I m\left\{e_{\mathcal{M}}(\tilde{u})\right\}$ & Rate & $\operatorname{Im}\left\{\tilde{e}_{\mathcal{M}}(\tilde{u}, \tilde{\phi})\right\}$ & Rate & $\operatorname{Im}\{a c c(\tilde{u}, \tilde{\phi})\}$ & Rate \\
\hline 35 & 30 & $8.6160 \mathrm{e}-04$ & & $8.5516 \mathrm{e}-04$ & & $6.4388 \mathrm{e}-06$ & \\
70 & 60 & $2.5912 \mathrm{e}-04$ & 1.733 & $2.5882 \mathrm{e}-04$ & 1.724 & $2.9318 \mathrm{e}-07$ & 4.457 \\
140 & 120 & $6.9310 \mathrm{e}-05$ & 1.902 & $6.9294 \mathrm{e}-05$ & 1.901 & $1.5930 \mathrm{e}-08$ & 4.202 \\
280 & 240 & $1.7836 \mathrm{e}-05$ & 1.958 & $1.7835 \mathrm{e}-05$ & 1.958 & $9.3912 \mathrm{e}-10$ & 4.084 \\
560 & 480 & $4.5193 \mathrm{e}-06$ & 1.981 & $4.5192 \mathrm{e}-06$ & 1.981 & $5.7240 \mathrm{e}-11$ & 4.036 \\
1120 & 960 & $1.1371 \mathrm{e}-06$ & 1.991 & $1.1371 \mathrm{e}-06$ & 1.991 & $3.5376 \mathrm{e}-12$ & 4.016 \\
\hline
\end{tabular}

4.1.4. Some results using a $C^{7}$ initial condition. The behavior in these tests seems to be the same as with the smooth initial condition. The tests are included here for reference. The similarity of these tests with the case where the initial condition is smooth is not surprising, since the methods we are looking at do not distinguish between a $C^{7}$ and smooth function. 
TABLE 28. Test: IC2 - Q1 - FS1 R1 - AS1 R1

\begin{tabular}{|r|r|rc|rc|rc|}
\hline Time steps & Cells & $\operatorname{Re}\left\{e_{\mathcal{M}}(\tilde{u})\right\}$ & Rate & $\operatorname{Re}\left\{\tilde{e}_{\mathcal{M}}(\tilde{u}, \tilde{\phi})\right\}$ & Rate & $\operatorname{Re}\{a c c(\tilde{u}, \tilde{\phi})\}$ & Rate \\
\hline 35 & 30 & $-1.1599 \mathrm{e}-02$ & & $-1.0218 \mathrm{e}-02$ & & $-1.3802 \mathrm{e}-03$ & \\
70 & 60 & $-5.9373 \mathrm{e}-03$ & 0.966 & $-5.6876 \mathrm{e}-03$ & 0.845 & $-2.4965 \mathrm{e}-04$ & 2.467 \\
140 & 120 & $-3.0039 \mathrm{e}-03$ & 0.983 & $-2.9545 \mathrm{e}-03$ & 0.945 & $-4.9404 \mathrm{e}-05$ & 2.337 \\
280 & 240 & $-1.5108 \mathrm{e}-03$ & 0.991 & $-1.5002 \mathrm{e}-03$ & 0.978 & $-1.0660 \mathrm{e}-05$ & 2.212 \\
560 & 480 & $-7.5764 \mathrm{e}-04$ & 0.996 & $-7.5519 \mathrm{e}-04$ & 0.990 & $-2.4499 \mathrm{e}-06$ & 2.121 \\
1120 & 960 & $-3.7938 \mathrm{e}-04$ & 0.998 & $-3.7879 \mathrm{e}-04$ & 0.995 & $-5.8532 \mathrm{e}-07$ & 2.065 \\
\hline \hline Time steps & Cells & $I m\left\{e_{\mathcal{M}}(\tilde{u})\right\}$ & Rate & $I m\left\{\tilde{e}_{\mathcal{M}}(\tilde{u}, \tilde{\phi})\right\}$ & Rate & $I m\{a c c(\tilde{u}, \tilde{\phi})\}$ & Rate \\
\hline 35 & 30 & $-5.9726 \mathrm{e}-04$ & & $-6.1595 \mathrm{e}-04$ & & $1.8688 \mathrm{e}-05$ & \\
70 & 60 & $-1.5054 \mathrm{e}-04$ & 1.988 & $-1.4827 \mathrm{e}-04$ & 2.055 & $-2.2729 \mathrm{e}-06$ & 3.039 \\
140 & 120 & $-3.7770 \mathrm{e}-05$ & 1.995 & $-3.7186 \mathrm{e}-05$ & 1.995 & $-5.8399 \mathrm{e}-07$ & 1.961 \\
280 & 240 & $-9.4581 \mathrm{e}-06$ & 1.998 & $-9.3660 \mathrm{e}-06$ & 1.989 & $-9.2057 \mathrm{e}-08$ & 2.665 \\
560 & 480 & $-2.3664 \mathrm{e}-06$ & 1.999 & $-2.3537 \mathrm{e}-06$ & 1.993 & $-1.2707 \mathrm{e}-08$ & 2.857 \\
1120 & 960 & $-5.9184 \mathrm{e}-07$ & 1.999 & $-5.9017 \mathrm{e}-07$ & 1.996 & $-1.6637 \mathrm{e}-09$ & 2.933 \\
\hline
\end{tabular}

TABle 29. Test: IC2 - Q1 - FS1 R1 - AS1 R2

\begin{tabular}{|r|r|rc|rc|rr|}
\hline Time steps & Cells & $\operatorname{Re}\left\{e_{\mathcal{M}}(\tilde{u})\right\}$ & Rate & $\operatorname{Re}\left\{\tilde{e}_{\mathcal{M}}(\tilde{u}, \tilde{\phi})\right\}$ & Rate & $\operatorname{Re}\{a c c(\tilde{u}, \tilde{\phi})\}$ & Rate \\
\hline 35 & 30 & $-1.1599 \mathrm{e}-02$ & & $-1.1049 \mathrm{e}-02$ & & $-5.4909 \mathrm{e}-04$ & \\
70 & 60 & $-5.9373 \mathrm{e}-03$ & 0.966 & $-5.7985 \mathrm{e}-03$ & 0.930 & $-1.3878 \mathrm{e}-04$ & 1.984 \\
140 & 120 & $-3.0039 \mathrm{e}-03$ & 0.983 & $-2.9687 \mathrm{e}-03$ & 0.966 & $-3.5163 \mathrm{e}-05$ & 1.981 \\
280 & 240 & $-1.5108 \mathrm{e}-03$ & 0.991 & $-1.5020 \mathrm{e}-03$ & 0.983 & $-8.8584 \mathrm{e}-06$ & 1.989 \\
560 & 480 & $-7.5764 \mathrm{e}-04$ & 0.996 & $-7.5542 \mathrm{e}-04$ & 0.992 & $-2.2233 \mathrm{e}-06$ & 1.994 \\
1120 & 960 & $-3.7938 \mathrm{e}-04$ & 0.998 & $-3.7882 \mathrm{e}-04$ & 0.996 & $-5.5691 \mathrm{e}-07$ & 1.997 \\
\hline \hline Time steps & Cells & $I m\left\{e_{\mathcal{M}}(\tilde{u})\right\}$ & Rate & $I m\left\{\tilde{e}_{\mathcal{M}}(\tilde{u}, \tilde{\phi})\right\}$ & Rate & $I m\{a c c(\tilde{u}, \tilde{\phi})\}$ & Rate \\
\hline 35 & 30 & $-5.9726 \mathrm{e}-04$ & & $-4.6168 \mathrm{e}-04$ & & $-1.3558 \mathrm{e}-04$ & \\
70 & 60 & $-1.5054 \mathrm{e}-04$ & 1.988 & $-1.3818 \mathrm{e}-04$ & 1.740 & $-1.2364 \mathrm{e}-05$ & 3.455 \\
140 & 120 & $-3.7770 \mathrm{e}-05$ & 1.995 & $-3.6543 \mathrm{e}-05$ & 1.919 & $-1.2271 \mathrm{e}-06$ & 3.333 \\
280 & 240 & $-9.4581 \mathrm{e}-06$ & 1.998 & $-9.3255 \mathrm{e}-06$ & 1.970 & $-1.3262 \mathrm{e}-07$ & 3.210 \\
560 & 480 & $-2.3664 \mathrm{e}-06$ & 1.999 & $-2.3512 \mathrm{e}-06$ & 1.988 & $-1.5253 \mathrm{e}-08$ & 3.120 \\
1120 & 960 & $-5.9184 \mathrm{e}-07$ & 1.999 & $-5.9001 \mathrm{e}-07$ & 1.995 & $-1.8231 \mathrm{e}-09$ & 3.065 \\
\hline
\end{tabular}


TABle 30. Test: IC2 - Q1 - FS1 R2 - AS1 R1

\begin{tabular}{|r|r|rc|rc|rc|}
\hline Time steps & Cells & $\operatorname{Re}\left\{e_{\mathcal{M}}(\tilde{u})\right\}$ & Rate & $\operatorname{Re}\left\{\tilde{e}_{\mathcal{M}}(\tilde{u}, \tilde{\phi})\right\}$ & Rate & $\operatorname{Re}\{a c c(\tilde{u}, \tilde{\phi})\}$ & Rate \\
\hline 35 & 30 & $-1.1598 \mathrm{e}-02$ & & $-1.1063 \mathrm{e}-02$ & & $-5.3463 \mathrm{e}-04$ & \\
70 & 60 & $-5.9372 \mathrm{e}-03$ & 0.966 & $-5.7989 \mathrm{e}-03$ & 0.932 & $-1.3831 \mathrm{e}-04$ & 1.951 \\
140 & 120 & $-3.0039 \mathrm{e}-03$ & 0.983 & $-2.9687 \mathrm{e}-03$ & 0.966 & $-3.5149 \mathrm{e}-05$ & 1.976 \\
280 & 240 & $-1.5108 \mathrm{e}-03$ & 0.991 & $-1.5020 \mathrm{e}-03$ & 0.983 & $-8.8579 \mathrm{e}-06$ & 1.988 \\
560 & 480 & $-7.5764 \mathrm{e}-04$ & 0.996 & $-7.5542 \mathrm{e}-04$ & 0.992 & $-2.2233 \mathrm{e}-06$ & 1.994 \\
1120 & 960 & $-3.7938 \mathrm{e}-04$ & 0.998 & $-3.7882 \mathrm{e}-04$ & 0.996 & $-5.5691 \mathrm{e}-07$ & 1.997 \\
\hline \hline Time steps & Cells & $I m\left\{e_{\mathcal{M}}(\tilde{u})\right\}$ & Rate & $I m\left\{\tilde{e}_{\mathcal{M}}(\tilde{u}, \tilde{\phi})\right\}$ & Rate & $I m\{a c c(\tilde{u}, \tilde{\phi})\}$ & Rate \\
\hline 35 & 30 & $-5.5248 \mathrm{e}-04$ & & $-5.4238 \mathrm{e}-04$ & & $-1.0104 \mathrm{e}-05$ & \\
70 & 60 & $-1.4465 \mathrm{e}-04$ & 1.933 & $-1.4060 \mathrm{e}-04$ & 1.948 & $-4.0528 \mathrm{e}-06$ & 1.318 \\
140 & 120 & $-3.7015 \mathrm{e}-05$ & 1.966 & $-3.6320 \mathrm{e}-05$ & 1.953 & $-6.9427 \mathrm{e}-07$ & 2.545 \\
280 & 240 & $-9.3625 \mathrm{e}-06$ & 1.983 & $-9.2636 \mathrm{e}-06$ & 1.971 & $-9.8914 \mathrm{e}-08$ & 2.811 \\
560 & 480 & $-2.3544 \mathrm{e}-06$ & 1.992 & $-2.3413 \mathrm{e}-06$ & 1.984 & $-1.3135 \mathrm{e}-08$ & 2.913 \\
1120 & 960 & $-5.9033 \mathrm{e}-07$ & 1.996 & $-5.8864 \mathrm{e}-07$ & 1.992 & $-1.6903 \mathrm{e}-09$ & 2.958 \\
\hline
\end{tabular}

TABle 31. Test: IC2 - Q1 - FS1 R2 - AS1 R2

\begin{tabular}{|r|r|rc|rc|rr|}
\hline Time steps & Cells & $\operatorname{Re}\left\{e_{\mathcal{M}}(\tilde{u})\right\}$ & Rate & $\operatorname{Re}\left\{\tilde{e}_{\mathcal{M}}(\tilde{u}, \tilde{\phi})\right\}$ & Rate & $\operatorname{Re}\{a c c(\tilde{u}, \tilde{\phi})\}$ & Rate \\
\hline 35 & 30 & $-1.1598 \mathrm{e}-02$ & & $-1.1058 \mathrm{e}-02$ & & $-5.3969 \mathrm{e}-04$ & \\
70 & 60 & $-5.9372 \mathrm{e}-03$ & 0.966 & $-5.7988 \mathrm{e}-03$ & 0.931 & $-1.3847 \mathrm{e}-04$ & 1.963 \\
140 & 120 & $-3.0039 \mathrm{e}-03$ & 0.983 & $-2.9687 \mathrm{e}-03$ & 0.966 & $-3.5154 \mathrm{e}-05$ & 1.978 \\
280 & 240 & $-1.5108 \mathrm{e}-03$ & 0.991 & $-1.5020 \mathrm{e}-03$ & 0.983 & $-8.8581 \mathrm{e}-06$ & 1.989 \\
560 & 480 & $-7.5764 \mathrm{e}-04$ & 0.996 & $-7.5542 \mathrm{e}-04$ & 0.992 & $-2.2233 \mathrm{e}-06$ & 1.994 \\
1120 & 960 & $-3.7938 \mathrm{e}-04$ & 0.998 & $-3.7882 \mathrm{e}-04$ & 0.996 & $-5.5691 \mathrm{e}-07$ & 1.997 \\
\hline \hline Time steps & Cells & $I m\left\{e_{\mathcal{M}}(\tilde{u})\right\}$ & Rate & $I m\left\{\tilde{e}_{\mathcal{M}}(\tilde{u}, \tilde{\phi})\right\}$ & Rate & $I m\{a c c(\tilde{u}, \tilde{\phi})\}$ & Rate \\
\hline 35 & 30 & $-5.5248 \mathrm{e}-04$ & & $-5.0100 \mathrm{e}-04$ & & $-5.1480 \mathrm{e}-05$ & \\
70 & 60 & $-1.4465 \mathrm{e}-04$ & 1.933 & $-1.3786 \mathrm{e}-04$ & 1.862 & $-6.7901 \mathrm{e}-06$ & 2.923 \\
140 & 120 & $-3.7015 \mathrm{e}-05$ & 1.966 & $-3.6145 \mathrm{e}-05$ & 1.931 & $-8.6965 \mathrm{e}-07$ & 2.965 \\
280 & 240 & $-9.3625 \mathrm{e}-06$ & 1.983 & $-9.2525 \mathrm{e}-06$ & 1.966 & $-1.1000 \mathrm{e}-07$ & 2.983 \\
560 & 480 & $-2.3544 \mathrm{e}-06$ & 1.992 & $-2.3406 \mathrm{e}-06$ & 1.983 & $-1.3831 \mathrm{e}-08$ & 2.992 \\
1120 & 960 & $-5.9033 \mathrm{e}-07$ & 1.996 & $-5.8859 \mathrm{e}-07$ & 1.992 & $-1.7340 \mathrm{e}-09$ & 2.996 \\
\hline
\end{tabular}


TABle 32. Test: IC2 - Q1 - FS1 R1 - AS2 R1

\begin{tabular}{|r|r|rc|rc|rc|}
\hline Time steps & Cells & $\operatorname{Re}\left\{e_{\mathcal{M}}(\tilde{u})\right\}$ & Rate & $\operatorname{Re}\left\{\tilde{e}_{\mathcal{M}}(\tilde{u}, \tilde{\phi})\right\}$ & Rate & $\operatorname{Re}\{a c c(\tilde{u}, \tilde{\phi})\}$ & Rate \\
\hline 35 & 30 & $-1.1599 \mathrm{e}-02$ & & $-1.0706 \mathrm{e}-02$ & & $-8.9239 \mathrm{e}-04$ & \\
70 & 60 & $-5.9373 \mathrm{e}-03$ & 0.966 & $-5.8229 \mathrm{e}-03$ & 0.879 & $-1.1438 \mathrm{e}-04$ & 2.964 \\
140 & 120 & $-3.0039 \mathrm{e}-03$ & 0.983 & $-2.9894 \mathrm{e}-03$ & 0.962 & $-1.4449 \mathrm{e}-05$ & 2.985 \\
280 & 240 & $-1.5108 \mathrm{e}-03$ & 0.991 & $-1.5090 \mathrm{e}-03$ & 0.986 & $-1.8147 \mathrm{e}-06$ & 2.993 \\
560 & 480 & $-7.5764 \mathrm{e}-04$ & 0.996 & $-7.5741 \mathrm{e}-04$ & 0.994 & $-2.2735 \mathrm{e}-07$ & 2.997 \\
1120 & 960 & $-3.7938 \mathrm{e}-04$ & 0.998 & $-3.7935 \mathrm{e}-04$ & 0.998 & $-2.8451 \mathrm{e}-08$ & 2.998 \\
\hline \hline Time steps & Cells & $I m\left\{e_{\mathcal{M}}(\tilde{u})\right\}$ & Rate & $I m\left\{\tilde{e}_{\mathcal{M}}(\tilde{u}, \tilde{\phi})\right\}$ & Rate & $I m\{a c c(\tilde{u}, \tilde{\phi})\}$ & Rate \\
\hline 35 & 30 & $-5.9726 \mathrm{e}-04$ & & $-6.8561 \mathrm{e}-04$ & & $8.8354 \mathrm{e}-05$ & \\
70 & 60 & $-1.5054 \mathrm{e}-04$ & 1.988 & $-1.5709 \mathrm{e}-04$ & 2.126 & $6.5463 \mathrm{e}-06$ & 3.755 \\
140 & 120 & $-3.7770 \mathrm{e}-05$ & 1.995 & $-3.8294 \mathrm{e}-05$ & 2.036 & $5.2414 \mathrm{e}-07$ & 3.643 \\
280 & 240 & $-9.4581 \mathrm{e}-06$ & 1.998 & $-9.5049 \mathrm{e}-06$ & 2.010 & $4.6779 \mathrm{e}-08$ & 3.486 \\
560 & 480 & $-2.3664 \mathrm{e}-06$ & 1.999 & $-2.3711 \mathrm{e}-06$ & 2.003 & $4.6661 \mathrm{e}-09$ & 3.326 \\
1120 & 960 & $-5.9184 \mathrm{e}-07$ & 1.999 & $-5.9234 \mathrm{e}-07$ & 2.001 & $5.0912 \mathrm{e}-10$ & 3.196 \\
\hline
\end{tabular}

TABle 33. Test: IC2 - Q1 - FS1 R1 - AS2 R2

\begin{tabular}{|r|r|rc|rc|rr|}
\hline Time steps & Cells & $\operatorname{Re}\left\{e_{\mathcal{M}}(\tilde{u})\right\}$ & Rate & $\operatorname{Re}\left\{\tilde{e}_{\mathcal{M}}(\tilde{u}, \tilde{\phi})\right\}$ & Rate & $\operatorname{Re}\{a c c(\tilde{u}, \tilde{\phi})\}$ & Rate \\
\hline 35 & 30 & $-1.1599 \mathrm{e}-02$ & & $-1.1578 \mathrm{e}-02$ & & $-2.0589 \mathrm{e}-05$ & \\
70 & 60 & $-5.9373 \mathrm{e}-03$ & 0.966 & $-5.9364 \mathrm{e}-03$ & 0.964 & $-8.5245 \mathrm{e}-07$ & 4.594 \\
140 & 120 & $-3.0039 \mathrm{e}-03$ & 0.983 & $-3.0039 \mathrm{e}-03$ & 0.983 & $-3.8966 \mathrm{e}-08$ & 4.451 \\
280 & 240 & $-1.5108 \mathrm{e}-03$ & 0.991 & $-1.5108 \mathrm{e}-03$ & 0.991 & $-1.9785 \mathrm{e}-09$ & 4.300 \\
560 & 480 & $-7.5764 \mathrm{e}-04$ & 0.996 & $-7.5764 \mathrm{e}-04$ & 0.996 & $-1.0925 \mathrm{e}-10$ & 4.179 \\
1120 & 960 & $-3.7938 \mathrm{e}-04$ & 0.998 & $-3.7938 \mathrm{e}-04$ & 0.998 & $-6.3794 \mathrm{e}-12$ & 4.098 \\
\hline \hline Time steps & Cells & $I m\left\{e_{\mathcal{M}}(\tilde{u})\right\}$ & Rate & $I m\left\{\tilde{e}_{\mathcal{M}}(\tilde{u}, \tilde{\phi})\right\}$ & Rate & $I m\{a c c(\tilde{u}, \tilde{\phi})\}$ & Rate \\
\hline 35 & 30 & $-5.9726 \mathrm{e}-04$ & & $-5.2703 \mathrm{e}-04$ & & $-7.0226 \mathrm{e}-05$ & \\
70 & 60 & $-1.5054 \mathrm{e}-04$ & 1.988 & $-1.4686 \mathrm{e}-04$ & 1.843 & $-3.6847 \mathrm{e}-06$ & 4.252 \\
140 & 120 & $-3.7770 \mathrm{e}-05$ & 1.995 & $-3.7646 \mathrm{e}-05$ & 1.964 & $-1.2345 \mathrm{e}-07$ & 4.900 \\
280 & 240 & $-9.4581 \mathrm{e}-06$ & 1.998 & $-9.4642 \mathrm{e}-06$ & 1.992 & $6.0800 \mathrm{e}-09$ & 4.344 \\
560 & 480 & $-2.3664 \mathrm{e}-06$ & 1.999 & $-2.3685 \mathrm{e}-06$ & 1.998 & $2.1158 \mathrm{e}-09$ & 1.523 \\
1120 & 960 & $-5.9184 \mathrm{e}-07$ & 1.999 & $-5.9219 \mathrm{e}-07$ & 2.000 & $3.4953 \mathrm{e}-10$ & 2.598 \\
\hline
\end{tabular}


TABle 34. Test: IC2 - Q1 - FS1 R2 - AS2 R1

\begin{tabular}{|r|r|rc|rc|rc|}
\hline Time steps & Cells & $\operatorname{Re}\left\{e_{\mathcal{M}}(\tilde{u})\right\}$ & Rate & $\operatorname{Re}\left\{\tilde{e}_{\mathcal{M}}(\tilde{u}, \tilde{\phi})\right\}$ & Rate & $\operatorname{Re}\{a c c(\tilde{u}, \tilde{\phi})\}$ & Rate \\
\hline 35 & 30 & $-1.1598 \mathrm{e}-02$ & & $-1.1592 \mathrm{e}-02$ & & $-5.6077 \mathrm{e}-06$ & \\
70 & 60 & $-5.9372 \mathrm{e}-03$ & 0.966 & $-5.9369 \mathrm{e}-03$ & 0.965 & $-3.7033 \mathrm{e}-07$ & 3.920 \\
140 & 120 & $-3.0039 \mathrm{e}-03$ & 0.983 & $-3.0039 \mathrm{e}-03$ & 0.983 & $-2.3719 \mathrm{e}-08$ & 3.965 \\
280 & 240 & $-1.5108 \mathrm{e}-03$ & 0.991 & $-1.5108 \mathrm{e}-03$ & 0.991 & $-1.4995 \mathrm{e}-09$ & 3.983 \\
560 & 480 & $-7.5764 \mathrm{e}-04$ & 0.996 & $-7.5764 \mathrm{e}-04$ & 0.996 & $-9.4239 \mathrm{e}-11$ & 3.992 \\
1120 & 960 & $-3.7938 \mathrm{e}-04$ & 0.998 & $-3.7938 \mathrm{e}-04$ & 0.998 & $-5.9084 \mathrm{e}-12$ & 3.995 \\
\hline \hline Time steps & Cells & $I m\left\{e_{\mathcal{M}}(\tilde{u})\right\}$ & Rate & $I m\left\{\tilde{e}_{\mathcal{M}}(\tilde{u}, \tilde{\phi})\right\}$ & Rate & $I m\{a c c(\tilde{u}, \tilde{\phi})\}$ & Rate \\
\hline 35 & 30 & $-5.5248 \mathrm{e}-04$ & & $-6.0947 \mathrm{e}-04$ & & $5.6987 \mathrm{e}-05$ & \\
70 & 60 & $-1.4465 \mathrm{e}-04$ & 1.933 & $-1.4919 \mathrm{e}-04$ & 2.030 & $4.5424 \mathrm{e}-06$ & 3.649 \\
140 & 120 & $-3.7015 \mathrm{e}-05$ & 1.966 & $-3.7412 \mathrm{e}-05$ & 1.996 & $3.9780 \mathrm{e}-07$ & 3.513 \\
280 & 240 & $-9.3625 \mathrm{e}-06$ & 1.983 & $-9.4014 \mathrm{e}-06$ & 1.993 & $3.8853 \mathrm{e}-08$ & 3.356 \\
560 & 480 & $-2.3544 \mathrm{e}-06$ & 1.992 & $-2.3586 \mathrm{e}-06$ & 1.995 & $4.1698 \mathrm{e}-09$ & 3.220 \\
1120 & 960 & $-5.9033 \mathrm{e}-07$ & 1.996 & $-5.9081 \mathrm{e}-07$ & 1.997 & $4.7807 \mathrm{e}-10$ & 3.125 \\
\hline
\end{tabular}

TABle 35. Test: IC2 - Q1 - FS1 R2 - AS2 R2

\begin{tabular}{|r|r|rc|rc|rr|}
\hline Time steps & Cells & $\operatorname{Re}\left\{e_{\mathcal{M}}(\tilde{u})\right\}$ & Rate & $\operatorname{Re}\left\{\tilde{e}_{\mathcal{M}}(\tilde{u}, \tilde{\phi})\right\}$ & Rate & $\operatorname{Re}\{a c c(\tilde{u}, \tilde{\phi})\}$ & Rate \\
\hline 35 & 30 & $-1.1598 \mathrm{e}-02$ & & $-1.1587 \mathrm{e}-02$ & & $-1.0756 \mathrm{e}-05$ & \\
70 & 60 & $-5.9372 \mathrm{e}-03$ & 0.966 & $-5.9367 \mathrm{e}-03$ & 0.965 & $-5.3603 \mathrm{e}-07$ & 4.327 \\
140 & 120 & $-3.0039 \mathrm{e}-03$ & 0.983 & $-3.0039 \mathrm{e}-03$ & 0.983 & $-2.8959 \mathrm{e}-08$ & 4.210 \\
280 & 240 & $-1.5108 \mathrm{e}-03$ & 0.991 & $-1.5108 \mathrm{e}-03$ & 0.991 & $-1.6641 \mathrm{e}-09$ & 4.121 \\
560 & 480 & $-7.5764 \mathrm{e}-04$ & 0.996 & $-7.5764 \mathrm{e}-04$ & 0.996 & $-9.9387 \mathrm{e}-11$ & 4.066 \\
1120 & 960 & $-3.7938 \mathrm{e}-04$ & 0.998 & $-3.7938 \mathrm{e}-04$ & 0.998 & $-6.0514 \mathrm{e}-12$ & 4.038 \\
\hline \hline Time steps & Cells & $I m\left\{e_{\mathcal{M}}(\tilde{u})\right\}$ & Rate & $I m\left\{\tilde{e}_{\mathcal{M}}(\tilde{u}, \tilde{\phi})\right\}$ & Rate & $I m\{a c c(\tilde{u}, \tilde{\phi})\}$ & Rate \\
\hline 35 & 30 & $-5.5248 \mathrm{e}-04$ & & $-5.6607 \mathrm{e}-04$ & & $1.3590 \mathrm{e}-05$ & \\
70 & 60 & $-1.4465 \mathrm{e}-04$ & 1.933 & $-1.4639 \mathrm{e}-04$ & 1.951 & $1.7395 \mathrm{e}-06$ & 2.966 \\
140 & 120 & $-3.7015 \mathrm{e}-05$ & 1.966 & $-3.7235 \mathrm{e}-05$ & 1.975 & $2.2033 \mathrm{e}-07$ & 2.981 \\
280 & 240 & $-9.3625 \mathrm{e}-06$ & 1.983 & $-9.3902 \mathrm{e}-06$ & 1.987 & $2.7699 \mathrm{e}-08$ & 2.992 \\
560 & 480 & $-2.3544 \mathrm{e}-06$ & 1.992 & $-2.3579 \mathrm{e}-06$ & 1.994 & $3.4708 \mathrm{e}-09$ & 2.996 \\
1120 & 960 & $-5.9033 \mathrm{e}-07$ & 1.996 & $-5.9076 \mathrm{e}-07$ & 1.997 & $4.3434 \mathrm{e}-10$ & 2.998 \\
\hline
\end{tabular}


TABLE 36. Test: IC2 - Q1 - FS2 R1 - AS1 R1

\begin{tabular}{|r|r|rc|rc|rc|}
\hline Time steps & Cells & $\operatorname{Re}\left\{e_{\mathcal{M}}(\tilde{u})\right\}$ & Rate & $\operatorname{Re}\left\{\tilde{e}_{\mathcal{M}}(\tilde{u}, \tilde{\phi})\right\}$ & Rate & $\operatorname{Re}\{a c c(\tilde{u}, \tilde{\phi})\}$ & Rate \\
\hline 35 & 30 & $-1.2063 \mathrm{e}-04$ & & $7.7112 \mathrm{e}-04$ & & $-8.9175 \mathrm{e}-04$ & \\
70 & 60 & $-1.4880 \mathrm{e}-05$ & 3.019 & $9.9479 \mathrm{e}-05$ & 2.954 & $-1.1436 \mathrm{e}-04$ & 2.963 \\
140 & 120 & $-1.8449 \mathrm{e}-06$ & 3.012 & $1.2603 \mathrm{e}-05$ & 2.981 & $-1.4448 \mathrm{e}-05$ & 2.985 \\
280 & 240 & $-2.2959 \mathrm{e}-07$ & 3.006 & $1.5851 \mathrm{e}-06$ & 2.991 & $-1.8147 \mathrm{e}-06$ & 2.993 \\
560 & 480 & $-2.8633 \mathrm{e}-08$ & 3.003 & $1.9872 \mathrm{e}-07$ & 2.996 & $-2.2735 \mathrm{e}-07$ & 2.997 \\
1120 & 960 & $-3.5749 \mathrm{e}-09$ & 3.002 & $2.4876 \mathrm{e}-08$ & 2.998 & $-2.8451 \mathrm{e}-08$ & 2.998 \\
\hline \hline Time steps & Cells & $I m\left\{e_{\mathcal{M}}(\tilde{u})\right\}$ & Rate & $I m\left\{\tilde{e}_{\mathcal{M}}(\tilde{u}, \tilde{\phi})\right\}$ & Rate & $I m\{a c c(\tilde{u}, \tilde{\phi})\}$ & Rate \\
\hline 35 & 30 & $2.4561 \mathrm{e}-04$ & & $1.6598 \mathrm{e}-04$ & & $7.9624 \mathrm{e}-05$ & \\
70 & 60 & $6.9088 \mathrm{e}-05$ & 1.830 & $6.3108 \mathrm{e}-05$ & 1.395 & $5.9805 \mathrm{e}-06$ & 3.735 \\
140 & 120 & $1.8141 \mathrm{e}-05$ & 1.929 & $1.7652 \mathrm{e}-05$ & 1.838 & $4.8824 \mathrm{e}-07$ & 3.615 \\
280 & 240 & $4.6378 \mathrm{e}-06$ & 1.968 & $4.5933 \mathrm{e}-06$ & 1.942 & $4.4521 \mathrm{e}-08$ & 3.455 \\
560 & 480 & $1.1719 \mathrm{e}-06$ & 1.985 & $1.1674 \mathrm{e}-06$ & 1.976 & $4.5245 \mathrm{e}-09$ & 3.299 \\
1120 & 960 & $2.9451 \mathrm{e}-07$ & 1.992 & $2.9401 \mathrm{e}-07$ & 1.989 & $5.0025 \mathrm{e}-10$ & 3.177 \\
\hline
\end{tabular}

TABle 37. Test: IC2 - Q1 - FS2 R1 - AS1 R2

\begin{tabular}{|r|r|rc|rc|rr|}
\hline Time steps & Cells & $\operatorname{Re}\left\{e_{\mathcal{M}}(\tilde{u})\right\}$ & Rate & $\operatorname{Re}\left\{\tilde{e}_{\mathcal{M}}(\tilde{u}, \tilde{\phi})\right\}$ & Rate & $\operatorname{Re}\{a c c(\tilde{u}, \tilde{\phi})\}$ & Rate \\
\hline 35 & 30 & $-1.2063 \mathrm{e}-04$ & & $-1.0033 \mathrm{e}-04$ & & $-2.0297 \mathrm{e}-05$ & \\
70 & 60 & $-1.4880 \mathrm{e}-05$ & 3.019 & $-1.4037 \mathrm{e}-05$ & 2.837 & $-8.4293 \mathrm{e}-07$ & 4.590 \\
140 & 120 & $-1.8449 \mathrm{e}-06$ & 3.012 & $-1.8063 \mathrm{e}-06$ & 2.958 & $-3.8663 \mathrm{e}-08$ & 4.446 \\
280 & 240 & $-2.2959 \mathrm{e}-07$ & 3.006 & $-2.2762 \mathrm{e}-07$ & 2.988 & $-1.9689 \mathrm{e}-09$ & 4.295 \\
560 & 480 & $-2.8633 \mathrm{e}-08$ & 3.003 & $-2.8524 \mathrm{e}-08$ & 2.996 & $-1.0895 \mathrm{e}-10$ & 4.176 \\
1120 & 960 & $-3.5749 \mathrm{e}-09$ & 3.002 & $-3.5685 \mathrm{e}-09$ & 2.999 & $-6.3704 \mathrm{e}-12$ & 4.096 \\
\hline \hline Time steps & Cells & $I m\left\{e_{\mathcal{M}}(\tilde{u})\right\}$ & Rate & $I m\left\{\tilde{e}_{\mathcal{M}}(\tilde{u}, \tilde{\phi})\right\}$ & Rate & $I m\{a c c(\tilde{u}, \tilde{\phi})\}$ & Rate \\
\hline 35 & 30 & $2.4561 \mathrm{e}-04$ & & $3.2020 \mathrm{e}-04$ & & $-7.4594 \mathrm{e}-05$ & \\
70 & 60 & $6.9088 \mathrm{e}-05$ & 1.830 & $7.3056 \mathrm{e}-05$ & 2.132 & $-3.9677 \mathrm{e}-06$ & 4.233 \\
140 & 120 & $1.8141 \mathrm{e}-05$ & 1.929 & $1.8282 \mathrm{e}-05$ & 1.999 & $-1.4140 \mathrm{e}-07$ & 4.810 \\
280 & 240 & $4.6378 \mathrm{e}-06$ & 1.968 & $4.6329 \mathrm{e}-06$ & 1.980 & $4.9507 \mathrm{e}-09$ & 4.836 \\
560 & 480 & $1.1719 \mathrm{e}-06$ & 1.985 & $1.1699 \mathrm{e}-06$ & 1.986 & $2.0450 \mathrm{e}-09$ & 1.276 \\
1120 & 960 & $2.9451 \mathrm{e}-07$ & 1.992 & $2.9417 \mathrm{e}-07$ & 1.992 & $3.4510 \mathrm{e}-10$ & 2.567 \\
\hline
\end{tabular}


TABle 38. Test: IC2 - Q1 - FS2 R2 - AS1 R1

\begin{tabular}{|r|r|rc|rc|rc|}
\hline Time steps & Cells & $\operatorname{Re}\left\{e_{\mathcal{M}}(\tilde{u})\right\}$ & Rate & $\operatorname{Re}\left\{\tilde{e}_{\mathcal{M}}(\tilde{u}, \tilde{\phi})\right\}$ & Rate & $\operatorname{Re}\{a c c(\tilde{u}, \tilde{\phi})\}$ & Rate \\
\hline 35 & 30 & $-1.2039 \mathrm{e}-04$ & & $-1.1514 \mathrm{e}-04$ & & $-5.2550 \mathrm{e}-06$ & \\
70 & 60 & $-1.4862 \mathrm{e}-05$ & 3.018 & $-1.4504 \mathrm{e}-05$ & 2.989 & $-3.5884 \mathrm{e}-07$ & 3.872 \\
140 & 120 & $-1.8437 \mathrm{e}-06$ & 3.011 & $-1.8203 \mathrm{e}-06$ & 2.994 & $-2.3353 \mathrm{e}-08$ & 3.942 \\
280 & 240 & $-2.2951 \mathrm{e}-07$ & 3.006 & $-2.2802 \mathrm{e}-07$ & 2.997 & $-1.4880 \mathrm{e}-09$ & 3.972 \\
560 & 480 & $-2.8627 \mathrm{e}-08$ & 3.003 & $-2.8534 \mathrm{e}-08$ & 2.998 & $-9.3877 \mathrm{e}-11$ & 3.986 \\
1120 & 960 & $-3.5745 \mathrm{e}-09$ & 3.002 & $-3.5686 \mathrm{e}-09$ & 2.999 & $-5.8971 \mathrm{e}-12$ & 3.993 \\
\hline \hline Time steps & Cells & $I m\left\{e_{\mathcal{M}}(\tilde{u})\right\}$ & Rate & $I m\left\{\tilde{e}_{\mathcal{M}}(\tilde{u}, \tilde{\phi})\right\}$ & Rate & $\operatorname{Im}\{a c c(\tilde{u}, \tilde{\phi})\}$ & Rate \\
\hline 35 & 30 & $2.9475 \mathrm{e}-04$ & & $2.4213 \mathrm{e}-04$ & & $5.2624 \mathrm{e}-05$ & \\
70 & 60 & $7.5267 \mathrm{e}-05$ & 1.969 & $7.1007 \mathrm{e}-05$ & 1.770 & $4.2595 \mathrm{e}-06$ & 3.627 \\
140 & 120 & $1.8914 \mathrm{e}-05$ & 1.993 & $1.8534 \mathrm{e}-05$ & 1.938 & $3.7985 \mathrm{e}-07$ & 3.487 \\
280 & 240 & $4.7345 \mathrm{e}-06$ & 1.998 & $4.6968 \mathrm{e}-06$ & 1.980 & $3.7723 \mathrm{e}-08$ & 3.332 \\
560 & 480 & $1.1840 \mathrm{e}-06$ & 2.000 & $1.1799 \mathrm{e}-06$ & 1.993 & $4.0990 \mathrm{e}-09$ & 3.202 \\
1120 & 960 & $2.9602 \mathrm{e}-07$ & 2.000 & $2.9555 \mathrm{e}-07$ & 1.997 & $4.7364 \mathrm{e}-10$ & 3.113 \\
\hline
\end{tabular}

TABle 39. Test: IC2 - Q1 - FS2 R2 - AS1 R2

\begin{tabular}{|r|r|rc|rc|rr|}
\hline Time steps & Cells & $\operatorname{Re}\left\{e_{\mathcal{M}}(\tilde{u})\right\}$ & Rate & $\operatorname{Re}\left\{\tilde{e}_{\mathcal{M}}(\tilde{u}, \tilde{\phi})\right\}$ & Rate & $\operatorname{Re}\{a c c(\tilde{u}, \tilde{\phi})\}$ & Rate \\
\hline 35 & 30 & $-1.2039 \mathrm{e}-04$ & & $-1.0964 \mathrm{e}-04$ & & $-1.0756 \mathrm{e}-05$ & \\
70 & 60 & $-1.4862 \mathrm{e}-05$ & 3.018 & $-1.4326 \mathrm{e}-05$ & 2.936 & $-5.3603 \mathrm{e}-07$ & 4.327 \\
140 & 120 & $-1.8437 \mathrm{e}-06$ & 3.011 & $-1.8147 \mathrm{e}-06$ & 2.981 & $-2.8959 \mathrm{e}-08$ & 4.210 \\
280 & 240 & $-2.2951 \mathrm{e}-07$ & 3.006 & $-2.2785 \mathrm{e}-07$ & 2.994 & $-1.6641 \mathrm{e}-09$ & 4.121 \\
560 & 480 & $-2.8627 \mathrm{e}-08$ & 3.003 & $-2.8528 \mathrm{e}-08$ & 2.998 & $-9.9387 \mathrm{e}-11$ & 4.066 \\
1120 & 960 & $-3.5745 \mathrm{e}-09$ & 3.002 & $-3.5685 \mathrm{e}-09$ & 2.999 & $-6.0517 \mathrm{e}-12$ & 4.038 \\
\hline \hline Time steps & Cells & $I m\left\{e_{\mathcal{M}}(\tilde{u})\right\}$ & Rate & $\operatorname{Im}\left\{\tilde{e}_{\mathcal{M}}(\tilde{u}, \tilde{\phi})\right\}$ & Rate & $\operatorname{Im}\{a c c(\tilde{u}, \tilde{\phi})\}$ & Rate \\
\hline 35 & 30 & $2.9475 \mathrm{e}-04$ & & $2.8116 \mathrm{e}-04$ & & $1.3590 \mathrm{e}-05$ & \\
70 & 60 & $7.5267 \mathrm{e}-05$ & 1.969 & $7.3527 \mathrm{e}-05$ & 1.935 & $1.7395 \mathrm{e}-06$ & 2.966 \\
140 & 120 & $1.8914 \mathrm{e}-05$ & 1.993 & $1.8694 \mathrm{e}-05$ & 1.976 & $2.2033 \mathrm{e}-07$ & 2.981 \\
280 & 240 & $4.7345 \mathrm{e}-06$ & 1.998 & $4.7068 \mathrm{e}-06$ & 1.990 & $2.7699 \mathrm{e}-08$ & 2.992 \\
560 & 480 & $1.1840 \mathrm{e}-06$ & 2.000 & $1.1805 \mathrm{e}-06$ & 1.995 & $3.4708 \mathrm{e}-09$ & 2.996 \\
1120 & 960 & $2.9602 \mathrm{e}-07$ & 2.000 & $2.9559 \mathrm{e}-07$ & 1.998 & $4.3434 \mathrm{e}-10$ & 2.998 \\
\hline
\end{tabular}


TABLE 40. Test: IC2 - Q1 - FS2 R1 - AS2 R1

\begin{tabular}{|r|r|rc|rc|rc|}
\hline Time steps & Cells & $\operatorname{Re}\left\{e_{\mathcal{M}}(\tilde{u})\right\}$ & Rate & $\operatorname{Re}\left\{\tilde{e}_{\mathcal{M}}(\tilde{u}, \tilde{\phi})\right\}$ & Rate & $\operatorname{Re}\{a c c(\tilde{u}, \tilde{\phi})\}$ & Rate \\
\hline 35 & 30 & $-1.2063 \mathrm{e}-04$ & & $8.0701 \mathrm{e}-04$ & & $-9.2764 \mathrm{e}-04$ & \\
70 & 60 & $-1.4880 \mathrm{e}-05$ & 3.019 & $1.0177 \mathrm{e}-04$ & 2.987 & $-1.1665 \mathrm{e}-04$ & 2.991 \\
140 & 120 & $-1.8449 \mathrm{e}-06$ & 3.012 & $1.2748 \mathrm{e}-05$ & 2.997 & $-1.4593 \mathrm{e}-05$ & 2.999 \\
280 & 240 & $-2.2959 \mathrm{e}-07$ & 3.006 & $1.5942 \mathrm{e}-06$ & 2.999 & $-1.8238 \mathrm{e}-06$ & 3.000 \\
560 & 480 & $-2.8633 \mathrm{e}-08$ & 3.003 & $1.9929 \mathrm{e}-07$ & 3.000 & $-2.2792 \mathrm{e}-07$ & 3.000 \\
1120 & 960 & $-3.5749 \mathrm{e}-09$ & 3.002 & $2.4911 \mathrm{e}-08$ & 3.000 & $-2.8486 \mathrm{e}-08$ & 3.000 \\
\hline \hline Time steps & Cells & $I m\left\{e_{\mathcal{M}}(\tilde{u})\right\}$ & Rate & $I m\left\{\tilde{e}_{\mathcal{M}}(\tilde{u}, \tilde{\phi})\right\}$ & Rate & $I m\{a c c(\tilde{u}, \tilde{\phi})\}$ & Rate \\
\hline 35 & 30 & $2.4561 \mathrm{e}-04$ & & $1.7433 \mathrm{e}-04$ & & $7.1272 \mathrm{e}-05$ & \\
70 & 60 & $6.9088 \mathrm{e}-05$ & 1.830 & $6.4543 \mathrm{e}-05$ & 1.434 & $4.5451 \mathrm{e}-06$ & 3.971 \\
140 & 120 & $1.8141 \mathrm{e}-05$ & 1.929 & $1.7854 \mathrm{e}-05$ & 1.854 & $2.8642 \mathrm{e}-07$ & 3.988 \\
280 & 240 & $4.6378 \mathrm{e}-06$ & 1.968 & $4.6199 \mathrm{e}-06$ & 1.950 & $1.7967 \mathrm{e}-08$ & 3.995 \\
560 & 480 & $1.1719 \mathrm{e}-06$ & 1.985 & $1.1708 \mathrm{e}-06$ & 1.980 & $1.1249 \mathrm{e}-09$ & 3.998 \\
1120 & 960 & $2.9451 \mathrm{e}-07$ & 1.992 & $2.9444 \mathrm{e}-07$ & 1.991 & $7.0365 \mathrm{e}-11$ & 3.999 \\
\hline
\end{tabular}

TABle 41. Test: IC2 - Q1 - FS2 R1 - AS2 R2

\begin{tabular}{|r|r|rc|rc|rr|}
\hline Time steps & Cells & $\operatorname{Re}\left\{e_{\mathcal{M}}(\tilde{u})\right\}$ & Rate & $\operatorname{Re}\left\{\tilde{e}_{\mathcal{M}}(\tilde{u}, \tilde{\phi})\right\}$ & Rate & $\operatorname{Re}\{a c c(\tilde{u}, \tilde{\phi})\}$ & Rate \\
\hline 35 & 30 & $-1.2063 \mathrm{e}-04$ & & $-1.0640 \mathrm{e}-04$ & & $-1.4231 \mathrm{e}-05$ & \\
70 & 60 & $-1.4880 \mathrm{e}-05$ & 3.019 & $-1.4440 \mathrm{e}-05$ & 2.881 & $-4.4015 \mathrm{e}-07$ & 5.015 \\
140 & 120 & $-1.8449 \mathrm{e}-06$ & 3.012 & $-1.8318 \mathrm{e}-06$ & 2.979 & $-1.3125 \mathrm{e}-08$ & 5.068 \\
280 & 240 & $-2.2959 \mathrm{e}-07$ & 3.006 & $-2.2922 \mathrm{e}-07$ & 2.998 & $-3.6748 \mathrm{e}-10$ & 5.158 \\
560 & 480 & $-2.8633 \mathrm{e}-08$ & 3.003 & $-2.8624 \mathrm{e}-08$ & 3.001 & $-8.7892 \mathrm{e}-12$ & 5.386 \\
1120 & 960 & $-3.5749 \mathrm{e}-09$ & 3.002 & $-3.5747 \mathrm{e}-09$ & 3.001 & $-1.0955 \mathrm{e}-13$ & 6.326 \\
\hline \hline Time steps & Cells & $I m\left\{e_{\mathcal{M}}(\tilde{u})\right\}$ & Rate & $\operatorname{Im}\left\{\tilde{e}_{\mathcal{M}}(\tilde{u}, \tilde{\phi})\right\}$ & Rate & $I m\{a c c(\tilde{u}, \tilde{\phi})\}$ & Rate \\
\hline 35 & 30 & $2.4561 \mathrm{e}-04$ & & $3.3270 \mathrm{e}-04$ & & $-8.7095 \mathrm{e}-05$ & \\
70 & 60 & $6.9088 \mathrm{e}-05$ & 1.830 & $7.4625 \mathrm{e}-05$ & 2.156 & $-5.5369 \mathrm{e}-06$ & 3.975 \\
140 & 120 & $1.8141 \mathrm{e}-05$ & 1.929 & $1.8488 \mathrm{e}-05$ & 2.013 & $-3.4745 \mathrm{e}-07$ & 3.994 \\
280 & 240 & $4.6378 \mathrm{e}-06$ & 1.968 & $4.6596 \mathrm{e}-06$ & 1.988 & $-2.1736 \mathrm{e}-08$ & 3.999 \\
560 & 480 & $1.1719 \mathrm{e}-06$ & 1.985 & $1.1733 \mathrm{e}-06$ & 1.990 & $-1.3587 \mathrm{e}-09$ & 4.000 \\
1120 & 960 & $2.9451 \mathrm{e}-07$ & 1.992 & $2.9460 \mathrm{e}-07$ & 1.994 & $-8.4923 \mathrm{e}-11$ & 4.000 \\
\hline
\end{tabular}


TABLE 42. Test: IC2 - Q1 - FS2 R2 - AS2 R1

\begin{tabular}{|r|r|rc|rc|rc|}
\hline Time steps & Cells & $\operatorname{Re}\left\{e_{\mathcal{M}}(\tilde{u})\right\}$ & Rate & $\operatorname{Re}\left\{\tilde{e}_{\mathcal{M}}(\tilde{u}, \tilde{\phi})\right\}$ & Rate & $\operatorname{Re}\{a c c(\tilde{u}, \tilde{\phi})\}$ & Rate \\
\hline 35 & 30 & $-1.2039 \mathrm{e}-04$ & & $-1.2178 \mathrm{e}-04$ & & $1.3827 \mathrm{e}-06$ & \\
70 & 60 & $-1.4862 \mathrm{e}-05$ & 3.018 & $-1.4920 \mathrm{e}-05$ & 3.029 & $5.8013 \mathrm{e}-08$ & 4.575 \\
140 & 120 & $-1.8437 \mathrm{e}-06$ & 3.011 & $-1.8463 \mathrm{e}-06$ & 3.015 & $2.5606 \mathrm{e}-09$ & 4.502 \\
280 & 240 & $-2.2951 \mathrm{e}-07$ & 3.006 & $-2.2964 \mathrm{e}-07$ & 3.007 & $1.2419 \mathrm{e}-10$ & 4.366 \\
560 & 480 & $-2.8627 \mathrm{e}-08$ & 3.003 & $-2.8634 \mathrm{e}-08$ & 3.004 & $6.6017 \mathrm{e}-12$ & 4.234 \\
1120 & 960 & $-3.5745 \mathrm{e}-09$ & 3.002 & $-3.5749 \mathrm{e}-09$ & 3.002 & $3.7318 \mathrm{e}-13$ & 4.145 \\
\hline \hline Time steps & Cells & $I m\left\{e_{\mathcal{M}}(\tilde{u})\right\}$ & Rate & $\operatorname{Im}\left\{\tilde{e}_{\mathcal{M}}(\tilde{u}, \tilde{\phi})\right\}$ & Rate & $\operatorname{Im}\{a c c(\tilde{u}, \tilde{\phi})\}$ & Rate \\
\hline 35 & 30 & $2.9475 \mathrm{e}-04$ & & $2.5310 \mathrm{e}-04$ & & $4.1648 \mathrm{e}-05$ & \\
70 & 60 & $7.5267 \mathrm{e}-05$ & 1.969 & $7.2671 \mathrm{e}-05$ & 1.800 & $2.5960 \mathrm{e}-06$ & 4.004 \\
140 & 120 & $1.8914 \mathrm{e}-05$ & 1.993 & $1.8752 \mathrm{e}-05$ & 1.954 & $1.6179 \mathrm{e}-07$ & 4.004 \\
280 & 240 & $4.7345 \mathrm{e}-06$ & 1.998 & $4.7244 \mathrm{e}-06$ & 1.989 & $1.0094 \mathrm{e}-08$ & 4.003 \\
560 & 480 & $1.1840 \mathrm{e}-06$ & 2.000 & $1.1834 \mathrm{e}-06$ & 1.997 & $6.3029 \mathrm{e}-10$ & 4.001 \\
1120 & 960 & $2.9602 \mathrm{e}-07$ & 2.000 & $2.9599 \mathrm{e}-07$ & 1.999 & $3.9374 \mathrm{e}-11$ & 4.001 \\
\hline
\end{tabular}

TABle 43. Test: IC2 - Q1 - FS2 R2 - AS2 R2

\begin{tabular}{|r|r|rc|rc|rc|}
\hline Time steps & Cells & $\operatorname{Re}\left\{e_{\mathcal{M}}(\tilde{u})\right\}$ & Rate & $\operatorname{Re}\left\{\tilde{e}_{\mathcal{M}}(\tilde{u}, \tilde{\phi})\right\}$ & Rate & $\operatorname{Re}\{a c c(\tilde{u}, \tilde{\phi})\}$ & Rate \\
\hline 35 & 30 & $-1.2039 \mathrm{e}-04$ & & $-1.1616 \mathrm{e}-04$ & & $-4.2363 \mathrm{e}-06$ & \\
70 & 60 & $-1.4862 \mathrm{e}-05$ & 3.018 & $-1.4741 \mathrm{e}-05$ & 2.978 & $-1.2110 \mathrm{e}-07$ & 5.129 \\
140 & 120 & $-1.8437 \mathrm{e}-06$ & 3.011 & $-1.8406 \mathrm{e}-06$ & 3.002 & $-3.0762 \mathrm{e}-09$ & 5.299 \\
280 & 240 & $-2.2951 \mathrm{e}-07$ & 3.006 & $-2.2946 \mathrm{e}-07$ & 3.004 & $-5.2457 \mathrm{e}-11$ & 5.874 \\
560 & 480 & $-2.8627 \mathrm{e}-08$ & 3.003 & $-2.8629 \mathrm{e}-08$ & 3.003 & $1.0843 \mathrm{e}-12$ & 5.596 \\
1120 & 960 & $-3.5745 \mathrm{e}-09$ & 3.002 & $-3.5748 \mathrm{e}-09$ & 3.002 & $2.1877 \mathrm{e}-13$ & 2.309 \\
\hline \hline Time steps & Cells & $I m\left\{e_{\mathcal{M}}(\tilde{u})\right\}$ & Rate & $\operatorname{Im}\left\{\tilde{e}_{\mathcal{M}}(\tilde{u}, \tilde{\phi})\right\}$ & Rate & $\operatorname{Im}\{a c c(\tilde{u}, \tilde{\phi})\}$ & Rate \\
\hline 35 & 30 & $2.9475 \mathrm{e}-04$ & & $2.9402 \mathrm{e}-04$ & & $7.3251 \mathrm{e}-07$ & \\
70 & 60 & $7.5267 \mathrm{e}-05$ & 1.969 & $7.5251 \mathrm{e}-05$ & 1.966 & $1.5974 \mathrm{e}-08$ & 5.519 \\
140 & 120 & $1.8914 \mathrm{e}-05$ & 1.993 & $1.8914 \mathrm{e}-05$ & 1.992 & $3.8509 \mathrm{e}-10$ & 5.374 \\
280 & 240 & $4.7345 \mathrm{e}-06$ & 1.998 & $4.7345 \mathrm{e}-06$ & 1.998 & $1.0212 \mathrm{e}-11$ & 5.237 \\
560 & 480 & $1.1840 \mathrm{e}-06$ & 2.000 & $1.1840 \mathrm{e}-06$ & 2.000 & $2.9050 \mathrm{e}-13$ & 5.136 \\
1120 & 960 & $2.9602 \mathrm{e}-07$ & 2.000 & $2.9602 \mathrm{e}-07$ & 2.000 & $8.7600 \mathrm{e}-15$ & 5.051 \\
\hline
\end{tabular}


TABLE 44. Test: IC2 - Q1 - FS3 R1 - AS1 R1

\begin{tabular}{|r|r|rc|rc|rc|}
\hline Time steps & Cells & $\operatorname{Re}\left\{e_{\mathcal{M}}(\tilde{u})\right\}$ & Rate & $\operatorname{Re}\left\{\tilde{e}_{\mathcal{M}}(\tilde{u}, \tilde{\phi})\right\}$ & Rate & $\operatorname{Re}\{a c c(\tilde{u}, \tilde{\phi})\}$ & Rate \\
\hline 35 & 30 & $-4.1300 \mathrm{e}-03$ & & $-3.0676 \mathrm{e}-03$ & & $-1.0624 \mathrm{e}-03$ & \\
70 & 60 & $-1.1882 \mathrm{e}-03$ & 1.797 & $-1.0479 \mathrm{e}-03$ & 1.550 & $-1.4031 \mathrm{e}-04$ & 2.921 \\
140 & 120 & $-2.8641 \mathrm{e}-04$ & 2.053 & $-2.6875 \mathrm{e}-04$ & 1.963 & $-1.7662 \mathrm{e}-05$ & 2.990 \\
280 & 240 & $-6.7863 \mathrm{e}-05$ & 2.077 & $-6.5656 \mathrm{e}-05$ & 2.033 & $-2.2067 \mathrm{e}-06$ & 3.001 \\
560 & 480 & $-1.6641 \mathrm{e}-05$ & 2.028 & $-1.6365 \mathrm{e}-05$ & 2.004 & $-2.7586 \mathrm{e}-07$ & 3.000 \\
1120 & 960 & $-4.1239 \mathrm{e}-06$ & 2.013 & $-4.0895 \mathrm{e}-06$ & 2.001 & $-3.4487 \mathrm{e}-08$ & 3.000 \\
\hline \hline Time steps & Cells & $I m\left\{e_{\mathcal{M}}(\tilde{u})\right\}$ & Rate & $I m\left\{\tilde{e}_{\mathcal{M}}(\tilde{u}, \tilde{\phi})\right\}$ & Rate & $I m\{a c c(\tilde{u}, \tilde{\phi})\}$ & Rate \\
\hline 35 & 30 & $-3.0582 \mathrm{e}-04$ & & $-3.5337 \mathrm{e}-04$ & & $4.7554 \mathrm{e}-05$ & \\
70 & 60 & $-2.2720 \mathrm{e}-05$ & 3.751 & $-2.6355 \mathrm{e}-05$ & 3.745 & $3.6351 \mathrm{e}-06$ & 3.709 \\
140 & 120 & $1.4913 \mathrm{e}-05$ & 0.607 & $1.4487 \mathrm{e}-05$ & 0.863 & $4.2651 \mathrm{e}-07$ & 3.091 \\
280 & 240 & $3.7346 \mathrm{e}-06$ & 1.998 & $3.6968 \mathrm{e}-06$ & 1.970 & $3.7844 \mathrm{e}-08$ & 3.494 \\
560 & 480 & $1.0901 \mathrm{e}-06$ & 1.777 & $1.0859 \mathrm{e}-06$ & 1.767 & $4.1617 \mathrm{e}-09$ & 3.185 \\
1120 & 960 & $2.8554 \mathrm{e}-07$ & 1.933 & $2.8506 \mathrm{e}-07$ & 1.930 & $4.7729 \mathrm{e}-10$ & 3.124 \\
\hline
\end{tabular}

TABLE 45. Test: IC2 - Q1 - FS3 R1 - AS1 R2

\begin{tabular}{|r|r|rc|rc|rr|}
\hline Time steps & Cells & $\operatorname{Re}\left\{e_{\mathcal{M}}(\tilde{u})\right\}$ & Rate & $\operatorname{Re}\left\{\tilde{e}_{\mathcal{M}}(\tilde{u}, \tilde{\phi})\right\}$ & Rate & $\operatorname{Re}\{a c c(\tilde{u}, \tilde{\phi})\}$ & Rate \\
\hline 35 & 30 & $-4.1300 \mathrm{e}-03$ & & $-3.9249 \mathrm{e}-03$ & & $-2.0511 \mathrm{e}-04$ & \\
70 & 60 & $-1.1882 \mathrm{e}-03$ & 1.797 & $-1.1609 \mathrm{e}-03$ & 1.757 & $-2.7297 \mathrm{e}-05$ & 2.910 \\
140 & 120 & $-2.8641 \mathrm{e}-04$ & 2.053 & $-2.8314 \mathrm{e}-04$ & 2.036 & $-3.2679 \mathrm{e}-06$ & 3.062 \\
280 & 240 & $-6.7863 \mathrm{e}-05$ & 2.077 & $-6.7468 \mathrm{e}-05$ & 2.069 & $-3.9445 \mathrm{e}-07$ & 3.050 \\
560 & 480 & $-1.6641 \mathrm{e}-05$ & 2.028 & $-1.6593 \mathrm{e}-05$ & 2.024 & $-4.8630 \mathrm{e}-08$ & 3.020 \\
1120 & 960 & $-4.1239 \mathrm{e}-06$ & 2.013 & $-4.1179 \mathrm{e}-06$ & 2.011 & $-6.0432 \mathrm{e}-09$ & 3.008 \\
\hline \hline Time steps & Cells & $I m\left\{e_{\mathcal{M}}(\tilde{u})\right\}$ & Rate & $\operatorname{Im}\left\{\tilde{e}_{\mathcal{M}}(\tilde{u}, \tilde{\phi})\right\}$ & Rate & $I m\{a c c(\tilde{u}, \tilde{\phi})\}$ & Rate \\
\hline 35 & 30 & $-3.0582 \mathrm{e}-04$ & & $-1.9827 \mathrm{e}-04$ & & $-1.0755 \mathrm{e}-04$ & \\
70 & 60 & $-2.2720 \mathrm{e}-05$ & 3.751 & $-1.6362 \mathrm{e}-05$ & 3.599 & $-6.3572 \mathrm{e}-06$ & 4.081 \\
140 & 120 & $-1.4913 \mathrm{e}-05$ & 0.607 & $1.5117 \mathrm{e}-05$ & 0.114 & $-2.0425 \mathrm{e}-07$ & 4.960 \\
280 & 240 & $3.7346 \mathrm{e}-06$ & 1.998 & $3.7364 \mathrm{e}-06$ & 2.016 & $-1.7710 \mathrm{e}-09$ & 6.850 \\
560 & 480 & $1.0901 \mathrm{e}-06$ & 1.777 & $1.0884 \mathrm{e}-06$ & 1.779 & $1.6808 \mathrm{e}-09$ & 0.075 \\
1120 & 960 & $2.8554 \mathrm{e}-07$ & 1.933 & $2.8522 \mathrm{e}-07$ & 1.932 & $3.2209 \mathrm{e}-10$ & 2.384 \\
\hline
\end{tabular}


TABLe 46. Test: IC2 - Q1 - FS3 R2 - AS1 R1

\begin{tabular}{|r|r|rc|rc|rr|}
\hline Time steps & Cells & $\operatorname{Re}\left\{e_{\mathcal{M}}(\tilde{u})\right\}$ & Rate & $\operatorname{Re}\left\{\tilde{e}_{\mathcal{M}}(\tilde{u}, \tilde{\phi})\right\}$ & Rate & $\operatorname{Re}\{a c c(\tilde{u}, \tilde{\phi})\}$ & Rate \\
\hline 35 & 30 & $-4.1296 \mathrm{e}-03$ & & $-3.9393 \mathrm{e}-03$ & & $-1.9024 \mathrm{e}-04$ & \\
70 & 60 & $-1.1881 \mathrm{e}-03$ & 1.797 & $-1.1613 \mathrm{e}-03$ & 1.762 & $-2.6817 \mathrm{e}-05$ & 2.827 \\
140 & 120 & $-2.8641 \mathrm{e}-04$ & 2.053 & $-2.8315 \mathrm{e}-04$ & 2.036 & $-3.2526 \mathrm{e}-06$ & 3.043 \\
280 & 240 & $-6.7863 \mathrm{e}-05$ & 2.077 & $-6.7469 \mathrm{e}-05$ & 2.069 & $-3.9397 \mathrm{e}-07$ & 3.045 \\
560 & 480 & $-1.6641 \mathrm{e}-05$ & 2.028 & $-1.6593 \mathrm{e}-05$ & 2.024 & $-4.8615 \mathrm{e}-08$ & 3.019 \\
1120 & 960 & $-4.1239 \mathrm{e}-06$ & 2.013 & $-4.1179 \mathrm{e}-06$ & 2.011 & $-6.0428 \mathrm{e}-09$ & 3.008 \\
\hline \hline Time steps & Cells & $I m\left\{e_{\mathcal{M}}(\tilde{u})\right\}$ & Rate & $I m\left\{\tilde{e}_{\mathcal{M}}(\tilde{u}, \tilde{\phi})\right\}$ & Rate & $I m\{a c c(\tilde{u}, \tilde{\phi})\}$ & Rate \\
\hline 35 & 30 & $-2.5820 \mathrm{e}-04$ & & $-2.7723 \mathrm{e}-04$ & & $1.9034 \mathrm{e}-05$ & \\
70 & 60 & $-1.6597 \mathrm{e}-05$ & 3.959 & $-1.8485 \mathrm{e}-05$ & 3.907 & $1.8878 \mathrm{e}-06$ & 3.334 \\
140 & 120 & $1.5685 \mathrm{e}-05$ & 0.082 & $1.5367 \mathrm{e}-05$ & 0.267 & $3.1812 \mathrm{e}-07$ & 2.569 \\
280 & 240 & $3.8313 \mathrm{e}-06$ & 2.033 & $3.8002 \mathrm{e}-06$ & 2.016 & $3.1043 \mathrm{e}-08$ & 3.357 \\
560 & 480 & $1.1022 \mathrm{e}-06$ & 1.797 & $1.0984 \mathrm{e}-06$ & 1.791 & $3.7362 \mathrm{e}-09$ & 3.055 \\
1120 & 960 & $2.8705 \mathrm{e}-07$ & 1.941 & $2.8660 \mathrm{e}-07$ & 1.938 & $4.5068 \mathrm{e}-10$ & 3.051 \\
\hline
\end{tabular}

TABLE 47. Test: IC2 - Q1 - FS3 R2 - AS1 R2

\begin{tabular}{|r|r|rc|rc|rr|}
\hline Time steps & Cells & $\operatorname{Re}\left\{e_{\mathcal{M}}(\tilde{u})\right\}$ & Rate & $\operatorname{Re}\left\{\tilde{e}_{\mathcal{M}}(\tilde{u}, \tilde{\phi})\right\}$ & Rate & $\operatorname{Re}\{a c c(\tilde{u}, \tilde{\phi})\}$ & Rate \\
\hline 35 & 30 & $-4.1296 \mathrm{e}-03$ & & $-3.9340 \mathrm{e}-03$ & & $-1.9553 \mathrm{e}-04$ & \\
70 & 60 & $-1.1881 \mathrm{e}-03$ & 1.797 & $-1.1611 \mathrm{e}-03$ & 1.760 & $-2.6989 \mathrm{e}-05$ & 2.857 \\
140 & 120 & $-2.8641 \mathrm{e}-04$ & 2.053 & $-2.8315 \mathrm{e}-04$ & 2.036 & $-3.2582 \mathrm{e}-06$ & 3.050 \\
280 & 240 & $-6.7863 \mathrm{e}-05$ & 2.077 & $-6.7468 \mathrm{e}-05$ & 2.069 & $-3.9414 \mathrm{e}-07$ & 3.047 \\
560 & 480 & $-1.6641 \mathrm{e}-05$ & 2.028 & $-1.6593 \mathrm{e}-05$ & 2.024 & $-4.8620 \mathrm{e}-08$ & 3.019 \\
1120 & 960 & $-4.1239 \mathrm{e}-06$ & 2.013 & $-4.1179 \mathrm{e}-06$ & 2.011 & $-6.0429 \mathrm{e}-09$ & 3.008 \\
\hline \hline Time steps & Cells & $I m\left\{e_{\mathcal{M}}(\tilde{u})\right\}$ & Rate & $I m\left\{\tilde{e}_{\mathcal{M}}(\tilde{u}, \tilde{\phi})\right\}$ & Rate & $I m\{a c c(\tilde{u}, \tilde{\phi})\}$ & Rate \\
\hline 35 & 30 & $-2.5820 \mathrm{e}-04$ & & $-2.3739 \mathrm{e}-04$ & & $-2.0811 \mathrm{e}-05$ & \\
70 & 60 & $-1.6597 \mathrm{e}-05$ & 3.959 & $-1.5922 \mathrm{e}-05$ & 3.898 & $-6.7563 \mathrm{e}-07$ & 4.945 \\
140 & 120 & $1.5685 \mathrm{e}-05$ & 0.082 & $1.5528 \mathrm{e}-05$ & 0.036 & $1.5709 \mathrm{e}-07$ & 2.105 \\
280 & 240 & $3.8313 \mathrm{e}-06$ & 2.033 & $3.8103 \mathrm{e}-06$ & 2.027 & $2.0971 \mathrm{e}-08$ & 2.905 \\
560 & 480 & $1.1022 \mathrm{e}-06$ & 1.797 & $1.0991 \mathrm{e}-06$ & 1.794 & $3.1066 \mathrm{e}-09$ & 2.755 \\
1120 & 960 & $2.8705 \mathrm{e}-07$ & 1.941 & $2.8664 \mathrm{e}-07$ & 1.939 & $4.1132 \mathrm{e}-10$ & 2.917 \\
\hline
\end{tabular}


TABLE 48. Test: IC2 - Q1 - FS3 R1 - AS2 R1

\begin{tabular}{|r|r|rc|rc|rr|}
\hline Time steps & Cells & $\operatorname{Re}\left\{e_{\mathcal{M}}(\tilde{u})\right\}$ & Rate & $\operatorname{Re}\left\{\tilde{e}_{\mathcal{M}}(\tilde{u}, \tilde{\phi})\right\}$ & Rate & $\operatorname{Re}\{a c c(\tilde{u}, \tilde{\phi})\}$ & Rate \\
\hline 35 & 30 & $-4.1300 \mathrm{e}-03$ & & $-3.2144 \mathrm{e}-03$ & & $-9.1560 \mathrm{e}-04$ & \\
70 & 60 & $-1.1882 \mathrm{e}-03$ & 1.797 & $-1.0719 \mathrm{e}-03$ & 1.584 & $-1.1623 \mathrm{e}-04$ & 2.978 \\
140 & 120 & $-2.8641 \mathrm{e}-04$ & 2.053 & $-2.7183 \mathrm{e}-04$ & 1.979 & $-1.4579 \mathrm{e}-05$ & 2.995 \\
280 & 240 & $-6.7863 \mathrm{e}-05$ & 2.077 & $-6.6039 \mathrm{e}-05$ & 2.041 & $-1.8234 \mathrm{e}-06$ & 2.999 \\
560 & 480 & $-1.6641 \mathrm{e}-05$ & 2.028 & $-1.6413 \mathrm{e}-05$ & 2.008 & $-2.2791 \mathrm{e}-07$ & 3.000 \\
1120 & 960 & $-4.1239 \mathrm{e}-06$ & 2.013 & $-4.0955 \mathrm{e}-06$ & 2.003 & $-2.8486 \mathrm{e}-08$ & 3.000 \\
\hline \hline Time steps & Cells & $I m\left\{e_{\mathcal{M}}(\tilde{u})\right\}$ & Rate & $I m\left\{\tilde{e}_{\mathcal{M}}(\tilde{u}, \tilde{\phi})\right\}$ & Rate & $I m\{a c c(\tilde{u}, \tilde{\phi})\}$ & Rate \\
\hline 35 & 30 & $-3.0582 \mathrm{e}-04$ & & $-3.8386 \mathrm{e}-04$ & & $7.8043 \mathrm{e}-05$ & \\
70 & 60 & $-2.2720 \mathrm{e}-05$ & 3.751 & $-2.7664 \mathrm{e}-05$ & 3.795 & $4.9439 \mathrm{e}-06$ & 3.981 \\
140 & 120 & $1.4913 \mathrm{e}-05$ & 0.607 & $1.4605 \mathrm{e}-05$ & 0.922 & $3.0816 \mathrm{e}-07$ & 4.004 \\
280 & 240 & $3.7346 \mathrm{e}-06$ & 1.998 & $3.7154 \mathrm{e}-06$ & 1.975 & $1.9245 \mathrm{e}-08$ & 4.001 \\
560 & 480 & $1.0901 \mathrm{e}-06$ & 1.777 & $1.0889 \mathrm{e}-06$ & 1.771 & $1.2022 \mathrm{e}-09$ & 4.001 \\
1120 & 960 & $2.8554 \mathrm{e}-07$ & 1.933 & $2.8546 \mathrm{e}-07$ & 1.931 & $7.5121 \mathrm{e}-11$ & 4.000 \\
\hline
\end{tabular}

TABle 49. Test: IC2 - Q1 - FS3 R1 - AS2 R2

\begin{tabular}{|r|r|rc|rc|rr|}
\hline Time steps & Cells & $\operatorname{Re}\left\{e_{\mathcal{M}}(\tilde{u})\right\}$ & Rate & $\operatorname{Re}\left\{\tilde{e}_{\mathcal{M}}(\tilde{u}, \tilde{\phi})\right\}$ & Rate & $\operatorname{Re}\{a c c(\tilde{u}, \tilde{\phi})\}$ & Rate \\
\hline 35 & 30 & $-4.1300 \mathrm{e}-03$ & & $-4.1132 \mathrm{e}-03$ & & $-1.6834 \mathrm{e}-05$ & \\
70 & 60 & $-1.1882 \mathrm{e}-03$ & 1.797 & $-1.1876 \mathrm{e}-03$ & 1.792 & $-5.2982 \mathrm{e}-07$ & 4.990 \\
140 & 120 & $-2.8641 \mathrm{e}-04$ & 2.053 & $-2.8639 \mathrm{e}-04$ & 2.052 & $-1.5265 \mathrm{e}-08$ & 5.117 \\
280 & 240 & $-6.7863 \mathrm{e}-05$ & 2.077 & $-6.7862 \mathrm{e}-05$ & 2.077 & $-4.4083 \mathrm{e}-10$ & 5.114 \\
560 & 480 & $-1.6641 \mathrm{e}-05$ & 2.028 & $-1.6641 \mathrm{e}-05$ & 2.028 & $-1.0961 \mathrm{e}-11$ & 5.330 \\
1120 & 960 & $-4.1239 \mathrm{e}-06$ & 2.013 & $-4.1239 \mathrm{e}-06$ & 2.013 & $-1.7694 \mathrm{e}-13$ & 5.953 \\
\hline \hline Time steps & Cells & $I m\left\{e_{\mathcal{M}}(\tilde{u})\right\}$ & Rate & $\operatorname{Im}\left\{\tilde{e}_{\mathcal{M}}(\tilde{u}, \tilde{\phi})\right\}$ & Rate & $I m\{a c c(\tilde{u}, \tilde{\phi})\}$ & Rate \\
\hline 35 & 30 & $-3.0582 \mathrm{e}-04$ & & $-2.2452 \mathrm{e}-04$ & & $-8.1296 \mathrm{e}-05$ & \\
70 & 60 & $-2.2720 \mathrm{e}-05$ & 3.751 & $-1.7536 \mathrm{e}-05$ & 3.678 & $-5.1835 \mathrm{e}-06$ & 3.971 \\
140 & 120 & $1.4913 \mathrm{e}-05$ & 0.607 & $1.5240 \mathrm{e}-05$ & 0.202 & $-3.2684 \mathrm{e}-07$ & 3.987 \\
280 & 240 & $3.7346 \mathrm{e}-06$ & 1.998 & $3.7551 \mathrm{e}-06$ & 2.021 & $-2.0503 \mathrm{e}-08$ & 3.995 \\
560 & 480 & $1.0901 \mathrm{e}-06$ & 1.777 & $1.0914 \mathrm{e}-06$ & 1.783 & $-1.2829 \mathrm{e}-09$ & 3.998 \\
1120 & 960 & $2.8554 \mathrm{e}-07$ & 1.933 & $2.8562 \mathrm{e}-07$ & 1.934 & $-8.0213 \mathrm{e}-11$ & 3.999 \\
\hline
\end{tabular}


TABLE 50. Test: IC2 - Q1 - FS3 R2 - AS2 R1

\begin{tabular}{|r|r|rc|rc|rc|}
\hline Time steps & Cells & $\operatorname{Re}\left\{e_{\mathcal{M}}(\tilde{u})\right\}$ & Rate & $\operatorname{Re}\left\{\tilde{e}_{\mathcal{M}}(\tilde{u}, \tilde{\phi})\right\}$ & Rate & $\operatorname{Re}\{a c c(\tilde{u}, \tilde{\phi})\}$ & Rate \\
\hline 35 & 30 & $-4.1296 \mathrm{e}-03$ & & $-4.1282 \mathrm{e}-03$ & & $-1.4123 \mathrm{e}-06$ & \\
70 & 60 & $-1.1881 \mathrm{e}-03$ & 1.797 & $-1.1881 \mathrm{e}-03$ & 1.797 & $-3.6051 \mathrm{e}-08$ & 5.292 \\
140 & 120 & $-2.8641 \mathrm{e}-04$ & 2.053 & $-2.8641 \mathrm{e}-04$ & 2.053 & $3.8479 \mathrm{e}-10$ & 6.550 \\
280 & 240 & $-6.7863 \mathrm{e}-05$ & 2.077 & $-6.7863 \mathrm{e}-05$ & 2.077 & $5.0073 \mathrm{e}-11$ & 2.942 \\
560 & 480 & $-1.6641 \mathrm{e}-05$ & 2.028 & $-1.6641 \mathrm{e}-05$ & 2.028 & $4.4212 \mathrm{e}-12$ & 3.502 \\
1120 & 960 & $-4.1239 \mathrm{e}-06$ & 2.013 & $-4.1239 \mathrm{e}-06$ & 2.013 & $3.0563 \mathrm{e}-13$ & 3.855 \\
\hline \hline Time steps & Cells & $I m\left\{e_{\mathcal{M}}(\tilde{u})\right\}$ & Rate & $I m\left\{\tilde{e}_{\mathcal{M}}(\tilde{u}, \tilde{\phi})\right\}$ & Rate & $I m\{a c c(\tilde{u}, \tilde{\phi})\}$ & Rate \\
\hline 35 & 30 & $-2.5820 \mathrm{e}-04$ & & $-3.0509 \mathrm{e}-04$ & & $4.6891 \mathrm{e}-05$ & \\
70 & 60 & $-1.6597 \mathrm{e}-05$ & 3.959 & $-1.9567 \mathrm{e}-05$ & 3.963 & $2.9691 \mathrm{e}-06$ & 3.981 \\
140 & 120 & $1.5685 \mathrm{e}-05$ & 0.082 & $1.5501 \mathrm{e}-05$ & 0.336 & $1.8355 \mathrm{e}-07$ & 4.016 \\
280 & 240 & $3.8313 \mathrm{e}-06$ & 2.033 & $3.8199 \mathrm{e}-06$ & 2.021 & $1.1369 \mathrm{e}-08$ & 4.013 \\
560 & 480 & $1.1022 \mathrm{e}-06$ & 1.797 & $1.1015 \mathrm{e}-06$ & 1.794 & $7.0754 \mathrm{e}-10$ & 4.006 \\
1120 & 960 & $2.8705 \mathrm{e}-07$ & 1.941 & $2.8701 \mathrm{e}-07$ & 1.940 & $4.4129 \mathrm{e}-11$ & 4.003 \\
\hline
\end{tabular}

TABle 51. Test: IC2 - Q1 - FS3 R2 - AS2 R2

\begin{tabular}{|r|r|rc|rc|rr|}
\hline Time steps & Cells & $\operatorname{Re}\left\{e_{\mathcal{M}}(\tilde{u})\right\}$ & Rate & $\operatorname{Re}\left\{\tilde{e}_{\mathcal{M}}(\tilde{u}, \tilde{\phi})\right\}$ & Rate & $\operatorname{Re}\{a c c(\tilde{u}, \tilde{\phi})\}$ & Rate \\
\hline 35 & 30 & $-4.1296 \mathrm{e}-03$ & & $-4.1228 \mathrm{e}-03$ & & $-6.8097 \mathrm{e}-06$ & \\
70 & 60 & $-1.1881 \mathrm{e}-03$ & 1.797 & $-1.1879 \mathrm{e}-03$ & 1.795 & $-2.1055 \mathrm{e}-07$ & 5.015 \\
140 & 120 & $-2.8641 \mathrm{e}-04$ & 2.053 & $-2.8640 \mathrm{e}-04$ & 2.052 & $-5.2249 \mathrm{e}-09$ & 5.333 \\
280 & 240 & $-6.7863 \mathrm{e}-05$ & 2.077 & $-6.7862 \mathrm{e}-05$ & 2.077 & $-1.2583 \mathrm{e}-10$ & 5.376 \\
560 & 480 & $-1.6641 \mathrm{e}-05$ & 2.028 & $-1.6641 \mathrm{e}-05$ & 2.028 & $-1.0877 \mathrm{e}-12$ & 6.854 \\
1120 & 960 & $-4.1239 \mathrm{e}-06$ & 2.013 & $-4.1239 \mathrm{e}-06$ & 2.013 & $1.5131 \mathrm{e}-13$ & 2.846 \\
\hline \hline Time steps & Cells & $I m\left\{e_{\mathcal{M}}(\tilde{u})\right\}$ & Rate & $I m\left\{\tilde{e}_{\mathcal{M}}(\tilde{u}, \tilde{\phi})\right\}$ & Rate & $I m\{a c c(\tilde{u}, \tilde{\phi})\}$ & Rate \\
\hline 35 & 30 & $-2.5820 \mathrm{e}-04$ & & $-2.6332 \mathrm{e}-04$ & & $5.1161 \mathrm{e}-06$ & \\
70 & 60 & $-1.6597 \mathrm{e}-05$ & 3.959 & $-1.6942 \mathrm{e}-05$ & 3.958 & $3.4463 \mathrm{e}-07$ & 3.892 \\
140 & 120 & $1.5685 \mathrm{e}-05$ & 0.082 & $1.5664 \mathrm{e}-05$ & 0.113 & $2.0623 \mathrm{e}-08$ & 4.063 \\
280 & 240 & $3.8313 \mathrm{e}-06$ & 2.033 & $3.8300 \mathrm{e}-06$ & 2.032 & $1.2371 \mathrm{e}-09$ & 4.059 \\
560 & 480 & $1.1022 \mathrm{e}-06$ & 1.797 & $1.1021 \mathrm{e}-06$ & 1.797 & $7.6035 \mathrm{e}-11$ & 4.024 \\
1120 & 960 & $2.8705 \mathrm{e}-07$ & 1.941 & $2.8705 \mathrm{e}-07$ & 1.941 & $4.7168 \mathrm{e}-12$ & 4.011 \\
\hline
\end{tabular}

4.1.5. Results using a $C^{1}$ initial condition. These results are the same in terms of convergence behavior as for the smooth initial conditions.

4.1.6. Results using an $H^{1}$ initial condition. It turns out these tests are also very similar, probably because the adjoint solution is still smooth. A few examples are provided here that illustrate an important consideration for adjoint error estimation. That is, given a forward solution that is not smooth, one can expect to get a good estimate of the error so long as the adjoint solution is sufficiently smooth. This can be seen in the proof of Theorem 3.1, since the errors in approximating the adjoint variable can be made small to account for larger errors in the approximation of the forward solution (since they multiply each other) occurring in the term $\operatorname{acc}(\tilde{u}, \tilde{\phi})$. 
Tables 52 - 54 provide evidence of this. These results show that the error in the imaginary part of the Fourier coefficient is not converging at the usual asymptotic rate of 2.0 observed in other tests (on these grids). However, the relative accuracy is about the same or better than in other similar tests. We will return to this point with different, non-smooth QOI's.

TABLE 52. Test: IC4 - Q1 - FS3 R1 - AS1 R1

\begin{tabular}{|r|r|rc|rc|rc|}
\hline Time steps & Cells & $\operatorname{Re}\left\{e_{\mathcal{M}}(\tilde{u})\right\}$ & Rate & $\operatorname{Re}\left\{\tilde{e}_{\mathcal{M}}(\tilde{u}, \tilde{\phi})\right\}$ & Rate & $\operatorname{Re}\{a c c(\tilde{u}, \tilde{\phi})\}$ & Rate \\
\hline 35 & 30 & $-3.0731 \mathrm{e}-03$ & & $-1.5607 \mathrm{e}-03$ & & $-1.5124 \mathrm{e}-03$ & \\
70 & 60 & $-7.5086 \mathrm{e}-04$ & 2.033 & $-5.5599 \mathrm{e}-04$ & 1.489 & $-1.9487 \mathrm{e}-04$ & 2.956 \\
140 & 120 & $-1.8093 \mathrm{e}-04$ & 2.053 & $-1.5631 \mathrm{e}-04$ & 1.831 & $-2.4620 \mathrm{e}-05$ & 2.985 \\
280 & 240 & $-4.4215 \mathrm{e}-05$ & 2.033 & $-4.1124 \mathrm{e}-05$ & 1.926 & $-3.0915 \mathrm{e}-06$ & 2.993 \\
560 & 480 & $-1.0925 \mathrm{e}-05$ & 2.017 & $-1.0538 \mathrm{e}-05$ & 1.964 & $-3.8733 \mathrm{e}-07$ & 2.997 \\
1120 & 960 & $-2.7192 \mathrm{e}-06$ & 2.006 & $-2.6707 \mathrm{e}-06$ & 1.980 & $-4.8482 \mathrm{e}-08$ & 2.998 \\
\hline \hline Time steps & Cells & $I m\left\{e_{\mathcal{M}}(\tilde{u})\right\}$ & Rate & $\operatorname{Im}\left\{\tilde{e}_{\mathcal{M}}(\tilde{u}, \tilde{\phi})\right\}$ & Rate & $\operatorname{Im}\{a c c(\tilde{u}, \tilde{\phi})\}$ & Rate \\
\hline 35 & 30 & $3.2973 \mathrm{e}-05$ & & $-7.1848 \mathrm{e}-05$ & & $1.0482 \mathrm{e}-04$ & \\
70 & 60 & $4.7168 \mathrm{e}-05$ & -0.517 & $3.9529 \mathrm{e}-05$ & 0.862 & $7.6392 \mathrm{e}-06$ & 3.778 \\
140 & 120 & $1.7856 \mathrm{e}-05$ & 1.401 & $1.7242 \mathrm{e}-05$ & 1.197 & $6.1395 \mathrm{e}-07$ & 3.637 \\
280 & 240 & $5.4552 \mathrm{e}-06$ & 1.711 & $5.3988 \mathrm{e}-06$ & 1.675 & $5.6440 \mathrm{e}-08$ & 3.443 \\
560 & 480 & $1.5459 \mathrm{e}-06$ & 1.819 & $1.5399 \mathrm{e}-06$ & 1.810 & $5.9513 \mathrm{e}-09$ & 3.245 \\
1120 & 960 & $4.2272 \mathrm{e}-07$ & 1.871 & $4.2202 \mathrm{e}-07$ & 1.867 & $6.9548 \mathrm{e}-10$ & 3.097 \\
\hline
\end{tabular}

TABle 53. Test: IC4 - Q1 - FS3 R2 - AS1 R1

\begin{tabular}{|r|r|rc|rc|rc|}
\hline Time steps & Cells & $\operatorname{Re}\left\{e_{\mathcal{M}}(\tilde{u})\right\}$ & Rate & $\operatorname{Re}\left\{\tilde{e}_{\mathcal{M}}(\tilde{u}, \tilde{\phi})\right\}$ & Rate & $\operatorname{Re}\{a c c(\tilde{u}, \tilde{\phi})\}$ & Rate \\
\hline 35 & 30 & $-3.0726 \mathrm{e}-03$ & & $-2.9394 \mathrm{e}-03$ & & $-1.3328 \mathrm{e}-04$ & \\
70 & 60 & $-7.5083 \mathrm{e}-04$ & 2.033 & $-7.3425 \mathrm{e}-04$ & 2.001 & $-1.6576 \mathrm{e}-05$ & 3.007 \\
140 & 120 & $-1.8092 \mathrm{e}-04$ & 2.053 & $-1.7889 \mathrm{e}-04$ & 2.037 & $-2.0305 \mathrm{e}-06$ & 3.029 \\
280 & 240 & $-4.4215 \mathrm{e}-05$ & 2.033 & $-4.3964 \mathrm{e}-05$ & 2.025 & $-2.5112 \mathrm{e}-07$ & 3.015 \\
560 & 480 & $-1.0925 \mathrm{e}-05$ & 2.017 & $-1.0894 \mathrm{e}-05$ & 2.013 & $-3.1294 \mathrm{e}-08$ & 3.004 \\
1120 & 960 & $-2.7192 \mathrm{e}-06$ & 2.006 & $-2.7153 \mathrm{e}-06$ & 2.004 & $-3.9187 \mathrm{e}-09$ & 2.997 \\
\hline \hline Time steps & Cells & $I m\left\{e_{\mathcal{M}}(\tilde{u})\right\}$ & Rate & $I m\left\{\tilde{e}_{\mathcal{M}}(\tilde{u}, \tilde{\phi})\right\}$ & Rate & $\operatorname{Im}\{a c c(\tilde{u}, \tilde{\phi})\}$ & Rate \\
\hline 35 & 30 & $1.0887 \mathrm{e}-04$ & & $4.7249 \mathrm{e}-05$ & & $6.1619 \mathrm{e}-05$ & \\
70 & 60 & $5.6812 \mathrm{e}-05$ & 0.938 & $5.1892 \mathrm{e}-05$ & -0.135 & $4.9209 \mathrm{e}-06$ & 3.646 \\
140 & 120 & $1.9066 \mathrm{e}-05$ & 1.575 & $1.8623 \mathrm{e}-05$ & 1.478 & $4.4359 \mathrm{e}-07$ & 3.472 \\
280 & 240 & $5.6067 \mathrm{e}-06$ & 1.766 & $5.5609 \mathrm{e}-06$ & 1.744 & $4.5778 \mathrm{e}-08$ & 3.276 \\
560 & 480 & $1.5648 \mathrm{e}-06$ & 1.841 & $1.5595 \mathrm{e}-06$ & 1.834 & $5.2844 \mathrm{e}-09$ & 3.115 \\
1120 & 960 & $4.2508 \mathrm{e}-07$ & 1.880 & $4.2443 \mathrm{e}-07$ & 1.878 & $6.5378 \mathrm{e}-10$ & 3.015 \\
\hline
\end{tabular}


TABle 54. Test: IC4 - Q1 - FS3 R2 - AS2 R2

\begin{tabular}{|r|r|rc|rc|rr|}
\hline Time steps & Cells & $\operatorname{Re}\left\{e_{\mathcal{M}}(\tilde{u})\right\}$ & Rate & $\operatorname{Re}\left\{\tilde{e}_{\mathcal{M}}(\tilde{u}, \tilde{\phi})\right\}$ & Rate & $\operatorname{Re}\{\operatorname{acc}(\tilde{u}, \tilde{\phi})\}$ & Rate \\
\hline 35 & 30 & $-3.0726 \mathrm{e}-03$ & & $-3.0643 \mathrm{e}-03$ & & $-8.3604 \mathrm{e}-06$ & \\
70 & 60 & $-7.5083 \mathrm{e}-04$ & 2.033 & $-7.5058 \mathrm{e}-04$ & 2.029 & $-2.4833 \mathrm{e}-07$ & 5.073 \\
140 & 120 & $-1.8092 \mathrm{e}-04$ & 2.053 & $-1.8092 \mathrm{e}-04$ & 2.053 & $-6.8646 \mathrm{e}-09$ & 5.177 \\
280 & 240 & $-4.4215 \mathrm{e}-05$ & 2.033 & $-4.4215 \mathrm{e}-05$ & 2.033 & $-1.5725 \mathrm{e}-10$ & 5.448 \\
560 & 480 & $-1.0925 \mathrm{e}-05$ & 2.017 & $-1.0925 \mathrm{e}-05$ & 2.017 & $-1.1101 \mathrm{e}-12$ & 7.146 \\
1120 & 960 & $-2.7192 \mathrm{e}-06$ & 2.006 & $-2.7192 \mathrm{e}-06$ & 2.006 & $2.4152 \mathrm{e}-13$ & 2.200 \\
\hline \hline Time steps & Cells & $I m\left\{e_{\mathcal{M}}(\tilde{u})\right\}$ & Rate & $\operatorname{Im}\left\{\tilde{e}_{\mathcal{M}}(\tilde{u}, \tilde{\phi})\right\}$ & Rate & $\operatorname{Im}\{a c c(\tilde{u}, \tilde{\phi})\}$ & Rate \\
\hline 35 & 30 & $1.0887 \mathrm{e}-04$ & & $1.0473 \mathrm{e}-04$ & & $4.1342 \mathrm{e}-06$ & \\
70 & 60 & $5.6812 \mathrm{e}-05$ & 0.938 & $5.6589 \mathrm{e}-05$ & 0.888 & $2.2361 \mathrm{e}-07$ & 4.209 \\
140 & 120 & $1.9066 \mathrm{e}-05$ & 1.575 & $1.9053 \mathrm{e}-05$ & 1.570 & $1.3049 \mathrm{e}-08$ & 4.099 \\
280 & 240 & $5.6067 \mathrm{e}-06$ & 1.766 & $5.6059 \mathrm{e}-06$ & 1.765 & $7.9287 \mathrm{e}-10$ & 4.041 \\
560 & 480 & $1.5648 \mathrm{e}-06$ & 1.841 & $1.5648 \mathrm{e}-06$ & 1.841 & $4.9059 \mathrm{e}-11$ & 4.014 \\
1120 & 960 & $4.2508 \mathrm{e}-07$ & 1.880 & $4.2508 \mathrm{e}-07$ & 1.880 & $3.0624 \mathrm{e}-12$ & 4.002 \\
\hline
\end{tabular}

4.1.7. Results using a discontinuous initial condition. Two results are provided here, in Tables 55 and 56. Strictly speaking, Theorem 3.1 does not apply here, due to the discontinuity in the forward solution. However, it is clear that after the first grid refinement is performed (then the discontinuities are on cell interfaces at the initial and final times), the imaginary part of the computable error estimate has an accuracy limited by the reconstruction used for the adjoint data. In Table 55 the accuracy converges to zero at rate 3 . This would be consistent with Theorem 3.1, if we could apply the theory, and shows that the piece-wise linear reconstruction of the adjoint data is suboptimal since the finite volume method used is second order. Indeed, in Table 56 a quadratic reconstruction is used and the accuracy of the computable error estimate is improved. Although the theory may not apply here directly as it stands, we may conclude from this example that in practice one should choose $p_{\phi} \geq s_{\phi}$, which is the same conclusion one should derive from Theorem 3.1 with smooth solutions.

TABLE 55. Test: IC5 - Q1 - FS2 R2 - AS2 R1

\begin{tabular}{|r|r|rc|rc|rr|}
\hline Time steps & Cells & $\operatorname{Re}\left\{e_{\mathcal{M}}(\tilde{u})\right\}$ & Rate & $\operatorname{Re}\left\{\tilde{e}_{\mathcal{M}}(\tilde{u}, \tilde{\phi})\right\}$ & Rate & $\operatorname{Re}\{\operatorname{acc}(\tilde{u}, \tilde{\phi})\}$ & Rate \\
\hline 35 & 30 & $-8.7975 \mathrm{e}-04$ & & $-8.7695 \mathrm{e}-04$ & & $-2.7994 \mathrm{e}-06$ & \\
70 & 60 & $2.5444 \mathrm{e}-04$ & 1.790 & $2.5362 \mathrm{e}-04$ & 1.790 & $8.2244 \mathrm{e}-07$ & 1.767 \\
140 & 120 & $6.8194 \mathrm{e}-05$ & 1.900 & $6.8146 \mathrm{e}-05$ & 1.896 & $4.7481 \mathrm{e}-08$ & 4.114 \\
280 & 240 & $1.7616 \mathrm{e}-05$ & 1.953 & $1.7613 \mathrm{e}-05$ & 1.952 & $2.8386 \mathrm{e}-09$ & 4.064 \\
560 & 480 & $4.4747 \mathrm{e}-06$ & 1.977 & $4.4745 \mathrm{e}-06$ & 1.977 & $1.7328 \mathrm{e}-10$ & 4.034 \\
1120 & 960 & $1.1275 \mathrm{e}-06$ & 1.989 & $1.1275 \mathrm{e}-06$ & 1.989 & $1.0693 \mathrm{e}-11$ & 4.018 \\
\hline \hline Time steps & Cells & $I m\left\{e_{\mathcal{M}}(\tilde{u})\right\}$ & Rate & $\operatorname{Im}\left\{\tilde{e}_{\mathcal{M}}(\tilde{u}, \tilde{\phi})\right\}$ & Rate & $\operatorname{Im}\{a c c(\tilde{u}, \tilde{\phi})\}$ & Rate \\
\hline 35 & 30 & $7.2403 \mathrm{e}-04$ & & $3.2372 \mathrm{e}-03$ & & $-2.5132 \mathrm{e}-03$ & \\
70 & 60 & $1.8540 \mathrm{e}-04$ & 1.965 & $1.9439 \mathrm{e}-04$ & 4.058 & $-8.9926 \mathrm{e}-06$ & 8.127 \\
140 & 120 & $4.6557 \mathrm{e}-05$ & 1.994 & $4.8073 \mathrm{e}-05$ & 2.016 & $-1.5159 \mathrm{e}-06$ & 2.569 \\
280 & 240 & $1.1652 \mathrm{e}-05$ & 1.998 & $1.1866 \mathrm{e}-05$ & 2.018 & $-2.1379 \mathrm{e}-07$ & 2.826 \\
560 & 480 & $2.9138 \mathrm{e}-06$ & 2.000 & $2.9420 \mathrm{e}-06$ & 2.012 & $-2.8238 \mathrm{e}-08$ & 2.921 \\
1120 & 960 & $7.2850 \mathrm{e}-07$ & 2.000 & $7.3213 \mathrm{e}-07$ & 2.007 & $-3.6242 \mathrm{e}-09$ & 2.962 \\
\hline
\end{tabular}


TABle 56. Test: IC5 - Q1 - FS2 R2 - AS2 R2

\begin{tabular}{|r|r|rc|rc|rr|}
\hline Time steps & Cells & $\operatorname{Re}\left\{e_{\mathcal{M}}(\tilde{u})\right\}$ & Rate & $\operatorname{Re}\left\{\tilde{e}_{\mathcal{M}}(\tilde{u}, \tilde{\phi})\right\}$ & Rate & $\operatorname{Re}\{a c c(\tilde{u}, \tilde{\phi})\}$ & Rate \\
\hline 35 & 30 & $-8.7975 \mathrm{e}-04$ & & $-8.6246 \mathrm{e}-04$ & & $-1.7297 \mathrm{e}-05$ & \\
70 & 60 & $2.5444 \mathrm{e}-04$ & 1.790 & $2.5412 \mathrm{e}-04$ & 1.763 & $3.1927 \mathrm{e}-07$ & 5.760 \\
140 & 120 & $6.8194 \mathrm{e}-05$ & 1.900 & $6.8164 \mathrm{e}-05$ & 1.898 & $3.0006 \mathrm{e}-08$ & 3.411 \\
280 & 240 & $1.7616 \mathrm{e}-05$ & 1.953 & $1.7614 \mathrm{e}-05$ & 1.952 & $2.1875 \mathrm{e}-09$ & 3.778 \\
560 & 480 & $4.4747 \mathrm{e}-06$ & 1.977 & $4.4746 \mathrm{e}-06$ & 1.977 & $1.4645 \mathrm{e}-10$ & 3.901 \\
1120 & 960 & $1.1275 \mathrm{e}-06$ & 1.989 & $1.1275 \mathrm{e}-06$ & 1.989 & $9.4936 \mathrm{e}-12$ & 3.947 \\
\hline \hline Time steps & Cells & $I m\left\{e_{\mathcal{M}}(\tilde{u})\right\}$ & Rate & $I m\left\{\tilde{e}_{\mathcal{M}}(\tilde{u}, \tilde{\phi})\right\}$ & Rate & $\operatorname{Im}\{a c c(\tilde{u}, \tilde{\phi})\}$ & Rate \\
\hline 35 & 30 & $7.2403 \mathrm{e}-04$ & & $3.4027 \mathrm{e}-03$ & & $-2.6787 \mathrm{e}-03$ & \\
70 & 60 & $1.8540 \mathrm{e}-04$ & 1.965 & $1.8553 \mathrm{e}-04$ & 4.197 & $-1.2968 \mathrm{e}-07$ & 14.334 \\
140 & 120 & $4.6557 \mathrm{e}-05$ & 1.994 & $4.6566 \mathrm{e}-05$ & 1.994 & $-9.6823 \mathrm{e}-09$ & 3.743 \\
280 & 240 & $1.1652 \mathrm{e}-05$ & 1.998 & $1.1653 \mathrm{e}-05$ & 1.999 & $-6.4028 \mathrm{e}-10$ & 3.919 \\
560 & 480 & $2.9138 \mathrm{e}-06$ & 2.000 & $2.9138 \mathrm{e}-06$ & 2.000 & $-4.0890 \mathrm{e}-11$ & 3.969 \\
1120 & 960 & $7.2850 \mathrm{e}-07$ & 2.000 & $7.2850 \mathrm{e}-07$ & 2.000 & $-2.5786 \mathrm{e}-12$ & 3.987 \\
\hline
\end{tabular}

4.2. Evaluation of the solution at a point. The quantity of interest is evaluation of $u\left(x=x^{*}, t=T\right)$, where $x^{*}=(1+\pi / 16) / 2 \in(1 / 2,1)$ is chosen to lie near the middle of $\Omega$ but is irrational, so that it will not lie at a cell center or cell interface. In this case the adjoint data is a distribution and the theory developed herein does not apply. In order to assess the practical limitations of using the computable error estimate proposed, some examples are studied here for this quantity of interest. This QOI could be considered a worst-case choice in terms of smoothness, since one expects most QOIs to correspond to adjoint data that is at least piece-wise smooth.

It should be noted that the solution value at a point can be approximated to arbitrary accuracy by integrating the solution against an appropriate Gaussian. In this case, the error should be reliably estimated by resolving the Gaussian with an appropriate grid. Alternatively, it may be worth instead using an $H^{1}$ hat function, since it is piece-wise linear and in theory can be approximated exactly using polynomials. This is not attempted in the current study.

4.2.1. Evaluation at a point with a smooth function. The results for this section are in Tables 57- 60. As expected the results are unpredictable, but it was observed here and in other tests that the error and the accuracy of the computable error estimate tend to be smaller using the higher order finite volume methods. In all the tests run, it was observed that the computable error estimate matched the true error in size, and usually the first 1-2 digits were correct. Also, most of the tests showed that the relative accuracy was generally improving as the grid was refined. 
TABLE 57. Test: IC1 - Q2 - FS1 R1 - AS1 R1

\begin{tabular}{|r|r|rr|rr|rr|}
\hline Time steps & Cells & $e_{\mathcal{M}}(\tilde{u})$ & Rate & $\tilde{e}_{\mathcal{M}}(\tilde{u}, \tilde{\phi})$ & Rate & $\operatorname{acc}(\tilde{u}, \tilde{\phi})$ & Rate \\
\hline 35 & 30 & $6.8336 \mathrm{e}-02$ & & $7.2395 \mathrm{e}-02$ & & $-4.0591 \mathrm{e}-03$ & \\
70 & 60 & $3.6448 \mathrm{e}-02$ & 0.907 & $3.7049 \mathrm{e}-02$ & 0.966 & $-6.0018 \mathrm{e}-04$ & 2.758 \\
140 & 120 & $1.8779 \mathrm{e}-02$ & 0.957 & $1.8760 \mathrm{e}-02$ & 0.982 & $1.8487 \mathrm{e}-05$ & 5.021 \\
280 & 240 & $9.5079 \mathrm{e}-03$ & 0.982 & $9.4420 \mathrm{e}-03$ & 0.991 & $6.5917 \mathrm{e}-05$ & -1.834 \\
560 & 480 & $4.7635 \mathrm{e}-03$ & 0.997 & $4.7368 \mathrm{e}-03$ & 0.995 & $2.6750 \mathrm{e}-05$ & 1.301 \\
1120 & 960 & $2.3902 \mathrm{e}-03$ & 0.995 & $2.3836 \mathrm{e}-03$ & 0.991 & $6.6572 \mathrm{e}-06$ & 2.007 \\
\hline
\end{tabular}

TABLE 58. Test: IC1 - Q2 - FS1 R1 - AS2 R2

\begin{tabular}{|r|r|rr|rr|rr|}
\hline Time steps & Cells & $e_{\mathcal{M}}(\tilde{u})$ & Rate & $\tilde{e}_{\mathcal{M}}(\tilde{u}, \tilde{\phi})$ & Rate & $a c c(\tilde{u}, \tilde{\phi})$ & Rate \\
\hline 35 & 30 & $6.8336 \mathrm{e}-02$ & & $7.5606 \mathrm{e}-02$ & & $-7.2699 \mathrm{e}-03$ & \\
70 & 60 & $3.6448 \mathrm{e}-02$ & 0.907 & $3.7900 \mathrm{e}-02$ & 0.996 & $-1.4518 \mathrm{e}-03$ & 2.324 \\
140 & 120 & $1.8779 \mathrm{e}-02$ & 0.957 & $1.8979 \mathrm{e}-02$ & 0.998 & $-2.0005 \mathrm{e}-04$ & 2.859 \\
280 & 240 & $9.5079 \mathrm{e}-03$ & 0.982 & $9.4973 \mathrm{e}-03$ & 0.999 & $1.0647 \mathrm{e}-05$ & 4.232 \\
560 & 480 & $4.7635 \mathrm{e}-03$ & 0.997 & $4.7507 \mathrm{e}-03$ & 0.999 & $1.2859 \mathrm{e}-05$ & -0.272 \\
1120 & 960 & $2.3902 \mathrm{e}-03$ & 0.995 & $2.3871 \mathrm{e}-03$ & 0.993 & $3.1590 \mathrm{e}-06$ & 2.025 \\
\hline
\end{tabular}

TABLE 59. Test: IC1 - Q2 - FS2 R2 - AS2 R2

\begin{tabular}{|r|r|rl|rr|rr|}
\hline Time steps & Cells & $e_{\mathcal{M}}(\tilde{u})$ & Rate & $\tilde{e}_{\mathcal{M}}(\tilde{u}, \tilde{\phi})$ & Rate & $a c c(\tilde{u}, \tilde{\phi})$ & Rate \\
\hline 35 & 30 & $1.6854 \mathrm{e}-03$ & & $1.9178 \mathrm{e}-03$ & & $-2.3242 \mathrm{e}-04$ & \\
70 & 60 & $3.8379 \mathrm{e}-04$ & 2.135 & $4.1263 \mathrm{e}-04$ & 2.217 & $-2.8844 \mathrm{e}-05$ & 3.010 \\
140 & 120 & $9.1664 \mathrm{e}-05$ & 2.066 & $9.4562 \mathrm{e}-05$ & 2.126 & $-2.8979 \mathrm{e}-06$ & 3.315 \\
280 & 240 & $2.2484 \mathrm{e}-05$ & 2.027 & $2.2575 \mathrm{e}-05$ & 2.067 & $-9.0864 \mathrm{e}-08$ & 4.995 \\
560 & 480 & $5.5661 \mathrm{e}-06$ & 2.014 & $5.5119 \mathrm{e}-06$ & 2.034 & $5.4230 \mathrm{e}-08$ & 0.745 \\
1120 & 960 & $1.3545 \mathrm{e}-06$ & 2.039 & $1.3495 \mathrm{e}-06$ & 2.030 & $5.0283 \mathrm{e}-09$ & 3.431 \\
\hline
\end{tabular}

TABle 60. Test: IC1 - Q2 - FS3 R2 - AS2 R2

\begin{tabular}{|r|r|rr|rr|rr|}
\hline Time steps & Cells & $e_{\mathcal{M}}(\tilde{u})$ & Rate & $\tilde{e}_{\mathcal{M}}(\tilde{u}, \tilde{\phi})$ & Rate & $a c c(\tilde{u}, \tilde{\phi})$ & Rate \\
\hline 35 & 30 & $-1.4330 \mathrm{e}-02$ & & $-1.6294 \mathrm{e}-02$ & & $1.9641 \mathrm{e}-03$ & \\
70 & 60 & $-1.6270 \mathrm{e}-04$ & 6.461 & $-3.0567 \mathrm{e}-04$ & 5.736 & $1.4296 \mathrm{e}-04$ & 3.780 \\
140 & 120 & $-5.7599 \mathrm{e}-04$ & -1.824 & $-6.3074 \mathrm{e}-04$ & -1.045 & $5.4746 \mathrm{e}-05$ & 1.385 \\
280 & 240 & $-3.0955 \mathrm{e}-05$ & 4.218 & $-2.5477 \mathrm{e}-05$ & 4.630 & $-5.4772 \mathrm{e}-06$ & 3.321 \\
560 & 480 & $-1.5485 \mathrm{e}-05$ & 0.999 & $-1.4801 \mathrm{e}-05$ & 0.784 & $-6.8365 \mathrm{e}-07$ & 3.002 \\
1120 & 960 & $-4.2803 \mathrm{e}-06$ & 1.855 & $-4.3062 \mathrm{e}-06$ & 1.781 & $2.5940 \mathrm{e}-08$ & 4.720 \\
\hline
\end{tabular}

4.2.2. Evaluation at a point with a $C^{7}$ function. In general the results here are similar to Section 4.2.1. A notable difference is that in some of the tests here the error in the solution value was not as well approximated. A typical example is shown in Table 62. The best results were observed when the adjoint solver was of higher order than the forward solver, e.g. Table 61 . It may be worth exploring if the results in Table 62 could be improved upon using a third order adjoint solver. 
TABle 61. Test: IC2 - Q2 - FS1 R1 - AS2 R2

\begin{tabular}{|r|r|rr|rr|rr|}
\hline Time steps & Cells & $e_{\mathcal{M}}(\tilde{u})$ & Rate & $\tilde{e}_{\mathcal{M}}(\tilde{u}, \tilde{\phi})$ & Rate & $a c c(\tilde{u}, \tilde{\phi})$ & Rate \\
\hline 35 & 30 & $-1.6239 \mathrm{e}-01$ & & $-3.6687 \mathrm{e}-02$ & & $-1.2570 \mathrm{e}-01$ & \\
70 & 60 & $-1.1384 \mathrm{e}-01$ & 0.512 & $-9.6592 \mathrm{e}-02$ & -1.397 & $-1.7245 \mathrm{e}-02$ & 2.866 \\
140 & 120 & $-7.0997 \mathrm{e}-02$ & 0.681 & $-6.9224 \mathrm{e}-02$ & 0.481 & $-1.7725 \mathrm{e}-03$ & 3.282 \\
280 & 240 & $-4.0357 \mathrm{e}-02$ & 0.815 & $-4.0186 \mathrm{e}-02$ & 0.785 & $-1.7088 \mathrm{e}-04$ & 3.375 \\
560 & 480 & $-2.1558 \mathrm{e}-02$ & 0.905 & $-2.1610 \mathrm{e}-02$ & 0.895 & $5.1724 \mathrm{e}-05$ & 1.724 \\
1120 & 960 & $-1.1186 \mathrm{e}-02$ & 0.947 & $-1.1189 \mathrm{e}-02$ & 0.950 & $3.7875 \mathrm{e}-06$ & 3.772 \\
\hline
\end{tabular}

TABLE 62. Test: IC2 - Q2 - FS2 R2 - AS2 R2

\begin{tabular}{|r|r|rr|rr|rr|}
\hline Time steps & Cells & $e_{\mathcal{M}}(\tilde{u})$ & Rate & $\tilde{e}_{\mathcal{M}}(\tilde{u}, \tilde{\phi})$ & Rate & $a c c(\tilde{u}, \tilde{\phi})$ & Rate \\
\hline 35 & 30 & $-5.4004 \mathrm{e}-02$ & & $8.0109 \mathrm{e}-03$ & & $-6.2015 \mathrm{e}-02$ & \\
70 & 60 & $-8.8903 \mathrm{e}-03$ & 2.603 & $-3.4946 \mathrm{e}-03$ & 1.197 & $-5.3956 \mathrm{e}-03$ & 3.523 \\
140 & 120 & $-1.2111 \mathrm{e}-03$ & 2.876 & $-9.3318 \mathrm{e}-04$ & 1.905 & $-2.7792 \mathrm{e}-04$ & 4.279 \\
280 & 240 & $-1.4110 \mathrm{e}-04$ & 3.102 & $-1.3385 \mathrm{e}-04$ & 2.802 & $-7.2478 \mathrm{e}-06$ & 5.261 \\
560 & 480 & $-1.3447 \mathrm{e}-05$ & 3.391 & $-1.7763 \mathrm{e}-05$ & 2.914 & $4.3162 \mathrm{e}-06$ & 0.748 \\
1120 & 960 & $-5.8330 \mathrm{e}-07$ & 4.527 & $-9.7294 \mathrm{e}-07$ & 4.190 & $3.8964 \mathrm{e}-07$ & 3.470 \\
\hline
\end{tabular}

4.2.3. Evaluation at a point with a $C^{1}$ function. No useful information was found in this case beyond that already presented in Sections 4.2.1-4.2.2.

4.2.4. Evaluation at a point with an $H^{1}$ function. The forward solution, being piece-wise linear, is represented very well using piece-wise linear (or better) reconstructions. Though the accuracy of the computable error estimate (Table 63) is poor relative to the size of the true error, the error is itself near roundoff after a few grid refinements.

TABLE 63. Test: IC4 - Q2 - FS2 R1 - AS1 R1

\begin{tabular}{|r|r|rr|rr|rr|}
\hline Time steps & Cells & $e_{\mathcal{M}}(\tilde{u})$ & Rate & $\tilde{e}_{\mathcal{M}}(\tilde{u}, \tilde{\phi})$ & Rate & $a c c(\tilde{u}, \tilde{\phi})$ & Rate \\
\hline 35 & 30 & $-6.8646 \mathrm{e}-03$ & & $2.0494 \mathrm{e}-02$ & & $-2.7359 \mathrm{e}-02$ & \\
70 & 60 & $5.3351 \mathrm{e}-04$ & 3.686 & $-2.6186 \mathrm{e}-03$ & 2.968 & $3.1521 \mathrm{e}-03$ & 3.118 \\
140 & 120 & $3.9475 \mathrm{e}-06$ & 7.078 & $-7.8088 \mathrm{e}-05$ & 5.068 & $8.2035 \mathrm{e}-05$ & 5.264 \\
280 & 240 & $-7.9709 \mathrm{e}-10$ & 12.274 & $-2.1432 \mathrm{e}-07$ & 8.509 & $2.1353 \mathrm{e}-07$ & 8.586 \\
560 & 480 & $1.3323 \mathrm{e}-15$ & 19.190 & $-2.4022 \mathrm{e}-12$ & 16.445 & $2.4035 \mathrm{e}-12$ & 16.439 \\
1120 & 960 & $3.1086 \mathrm{e}-15$ & -1.222 & $-9.7145 \mathrm{e}-17$ & 14.594 & $3.2058 \mathrm{e}-15$ & 9.550 \\
\hline
\end{tabular}

4.2.5. Evaluation of a discontinuous function at a point. These results were observed to be essentially the same in terms of convergence behavior and accuracy on fine grids as in Section 4.2.4.

4.3. Solution moment of order zero. This QOI is just a diagnostic, since the finite volume methods preserve the integral of the solution. Thus the error should be roundoff sized in all cases. Indeed this is what was observed. 
4.4. Solution moment of order one. The adjoint solution is discontinuous in this case since it is forced to be periodic in $x$, hence as $x$ varies from slightly less than 1 to slightly more than 1 the adjoint solution at the final time changes value from 1 to 0 instantaneously. In this case there is a different behavior observed between the linear and nonlinear methods for the forward problem. In Table 64 the forward and adjoint solvers are first order upwind. It is not clear what the asymptotic behavior of $\operatorname{acc}(\tilde{u}, \tilde{\phi})$ is in this case, but the data suggests that the true asymptotic behavior may not be represented over this (coarse) range of grid sizes. Comparing with Table 65, we see that at the finest grid size the accuracy of the computable error estimate is larger using the second order adjoint solver. However, the data also suggests the asymptotic behavior with the second order adjoint solver may be different at grid sizes beyond what is shown here. Thus no conclusion may be drawn without further investigation.

Tables 66-67 show the analogous results using the second order upwind method for the forward problem. In this case $\operatorname{acc}(\tilde{u}, \tilde{\phi})$ is smaller using the second order upwind method for the adjoint problem than using the first order upwind method. Based solely on the data shown it seems likely this would also be the case at smaller grid sizes.

Tables 68-69 display results using the nonlinear TVD method for the forward problem. The accuracy of the computable error estimate is poor relative to the size of the error in the quantity of interest. These results are also poor compared to the former tests using linear methods for the forward problem. These relatively poor results were observed also using linear reconstructions of the finite volume data for both forward and adjoint problems. In contrast to the tests in Sections 4.1-4.3, here the accuracy of the computable error estimate was not as good when the TVD method was used for the forward problem as when either of the linear methods was used.

While the discontinuity of the forward solution introduces a challenge for adjoint error estimation, it should be noted that in this section and in the next, the discontinuity is introduced in order to avoid extending the theory to include non-periodic boundary conditions. If this extension were made, the moments of the solution could potentially be represented using an adjoint problem with a more smooth solution. There is an adjoint analysis performed in the book of Marchuk, Agoshkov and Shutyaev [18] that handles non-periodic boundary conditions for hyperbolic conservation laws in $1 \mathrm{D}$.

TABLE 64. Test: IC1 - Q4 - FS1 R1 - AS1 R1

\begin{tabular}{|r|r|rr|rr|rr|}
\hline Time steps & Cells & $e_{\mathcal{M}}(\tilde{u})$ & Rate & $\tilde{e}_{\mathcal{M}}(\tilde{u}, \tilde{\phi})$ & Rate & $a c c(\tilde{u}, \tilde{\phi})$ & Rate \\
\hline 35 & 30 & $-6.7983 \mathrm{e}-04$ & & $-7.6068 \mathrm{e}-04$ & & $8.0848 \mathrm{e}-05$ & \\
70 & 60 & $-1.7799 \mathrm{e}-04$ & 1.933 & $-1.8261 \mathrm{e}-04$ & 2.059 & $4.6237 \mathrm{e}-06$ & 4.128 \\
140 & 120 & $-4.5545 \mathrm{e}-05$ & 1.966 & $-4.5767 \mathrm{e}-05$ & 1.996 & $2.2131 \mathrm{e}-07$ & 4.385 \\
280 & 240 & $-1.1520 \mathrm{e}-05$ & 1.983 & $-1.1525 \mathrm{e}-05$ & 1.989 & $4.9744 \mathrm{e}-09$ & 5.475 \\
560 & 480 & $-2.8970 \mathrm{e}-06$ & 1.992 & $-2.8962 \mathrm{e}-06$ & 1.993 & $-8.0808 \mathrm{e}-10$ & 2.622 \\
1120 & 960 & $-7.2638 \mathrm{e}-07$ & 1.996 & $-7.2619 \mathrm{e}-07$ & 1.996 & $-1.9074 \mathrm{e}-10$ & 2.083 \\
\hline
\end{tabular}


TABle 65. Test: IC1 - Q4 - FS1 R1 - AS2 R2

\begin{tabular}{|r|r|rr|rr|rr|}
\hline Time steps & Cells & $e_{\mathcal{M}}(\tilde{u})$ & Rate & $\tilde{e}_{\mathcal{M}}(\tilde{u}, \tilde{\phi})$ & Rate & $a c c(\tilde{u}, \tilde{\phi})$ & Rate \\
\hline 35 & 30 & $-6.7983 \mathrm{e}-04$ & & $-6.5087 \mathrm{e}-04$ & & $-2.8959 \mathrm{e}-05$ & \\
70 & 60 & $-1.7799 \mathrm{e}-04$ & 1.933 & $-1.8087 \mathrm{e}-04$ & 1.847 & $2.8849 \mathrm{e}-06$ & 3.327 \\
140 & 120 & $-4.5545 \mathrm{e}-05$ & 1.966 & $-4.6333 \mathrm{e}-05$ & 1.965 & $7.8812 \mathrm{e}-07$ & 1.872 \\
280 & 240 & $-1.1520 \mathrm{e}-05$ & 1.983 & $-1.1646 \mathrm{e}-05$ & 1.992 & $1.2574 \mathrm{e}-07$ & 2.648 \\
560 & 480 & $-2.8970 \mathrm{e}-06$ & 1.992 & $-2.9144 \mathrm{e}-06$ & 1.999 & $1.7431 \mathrm{e}-08$ & 2.851 \\
1120 & 960 & $-7.2638 \mathrm{e}-07$ & 1.996 & $-7.2867 \mathrm{e}-07$ & 2.000 & $2.2865 \mathrm{e}-09$ & 2.931 \\
\hline
\end{tabular}

TABLE 66. Test: IC1 - Q4 - FS2 R2 - AS1 R1

\begin{tabular}{|r|r|rr|rr|rr|}
\hline Time steps & Cells & $e_{\mathcal{M}}(\tilde{u})$ & Rate & $\tilde{e}_{\mathcal{M}}(\tilde{u}, \tilde{\phi})$ & Rate & $a c c(\tilde{u}, \tilde{\phi})$ & Rate \\
\hline 35 & 30 & $3.6269 \mathrm{e}-04$ & & $2.9902 \mathrm{e}-04$ & & $6.3672 \mathrm{e}-05$ & \\
70 & 60 & $9.2613 \mathrm{e}-05$ & 1.969 & $8.7452 \mathrm{e}-05$ & 1.774 & $5.1615 \mathrm{e}-06$ & 3.625 \\
140 & 120 & $2.3273 \mathrm{e}-05$ & 1.993 & $2.2811 \mathrm{e}-05$ & 1.939 & $4.6218 \mathrm{e}-07$ & 3.481 \\
280 & 240 & $5.8257 \mathrm{e}-06$ & 1.998 & $5.7796 \mathrm{e}-06$ & 1.981 & $4.6087 \mathrm{e}-08$ & 3.326 \\
560 & 480 & $1.4569 \mathrm{e}-06$ & 2.000 & $1.4519 \mathrm{e}-06$ & 1.993 & $5.0229 \mathrm{e}-09$ & 3.198 \\
1120 & 960 & $3.6425 \mathrm{e}-07$ & 2.000 & $3.6367 \mathrm{e}-07$ & 1.997 & $5.8150 \mathrm{e}-10$ & 3.111 \\
\hline
\end{tabular}

TABle 67. Test: IC1 - Q4 - FS2 R2 - AS2 R2

\begin{tabular}{|r|r|rc|rc|rr|}
\hline Time steps & Cells & $e_{\mathcal{M}}(\tilde{u})$ & Rate & $\tilde{e}_{\mathcal{M}}(\tilde{u}, \tilde{\phi})$ & Rate & $a c c(\tilde{u}, \tilde{\phi})$ & Rate \\
\hline 35 & 30 & $3.6269 \mathrm{e}-04$ & & $3.6311 \mathrm{e}-04$ & & $-4.1341 \mathrm{e}-07$ & \\
70 & 60 & $9.2613 \mathrm{e}-05$ & 1.969 & $9.2678 \mathrm{e}-05$ & 1.970 & $-6.4838 \mathrm{e}-08$ & 2.673 \\
140 & 120 & $2.3273 \mathrm{e}-05$ & 1.993 & $2.3278 \mathrm{e}-05$ & 1.993 & $-4.8411 \mathrm{e}-09$ & 3.743 \\
280 & 240 & $5.8257 \mathrm{e}-06$ & 1.998 & $5.8260 \mathrm{e}-06$ & 1.998 & $-3.2014 \mathrm{e}-10$ & 3.919 \\
560 & 480 & $1.4569 \mathrm{e}-06$ & 2.000 & $1.4569 \mathrm{e}-06$ & 2.000 & $-2.0445 \mathrm{e}-11$ & 3.969 \\
1120 & 960 & $3.6425 \mathrm{e}-07$ & 2.000 & $3.6425 \mathrm{e}-07$ & 2.000 & $-1.2899 \mathrm{e}-12$ & 3.986 \\
\hline
\end{tabular}

TABLE 68. Test: IC1 - Q4 - FS3 R2 - AS1 R1

\begin{tabular}{|r|r|rr|rr|rr|}
\hline Time steps & Cells & $e_{\mathcal{M}}(\tilde{u})$ & Rate & $\tilde{e}_{\mathcal{M}}(\tilde{u}, \tilde{\phi})$ & Rate & $a c c(\tilde{u}, \tilde{\phi})$ & Rate \\
\hline 35 & 30 & $-7.1250 \mathrm{e}-05$ & & $2.6581 \mathrm{e}-04$ & & $-3.3706 \mathrm{e}-04$ & \\
70 & 60 & $-3.4832 \mathrm{e}-06$ & 4.354 & $6.9189 \mathrm{e}-05$ & 1.942 & $-7.2672 \mathrm{e}-05$ & 2.214 \\
140 & 120 & $2.2199 \mathrm{e}-06$ & 0.650 & $1.7463 \mathrm{e}-05$ & 1.986 & $-1.5244 \mathrm{e}-05$ & 2.253 \\
280 & 240 & $1.1985 \mathrm{e}-06$ & 0.889 & $4.4705 \mathrm{e}-06$ & 1.966 & $-3.2721 \mathrm{e}-06$ & 2.220 \\
560 & 480 & $4.3131 \mathrm{e}-07$ & 1.474 & $1.1576 \mathrm{e}-06$ & 1.949 & $-7.2625 \mathrm{e}-07$ & 2.172 \\
1120 & 960 & $1.3456 \mathrm{e}-07$ & 1.681 & $3.0071 \mathrm{e}-07$ & 1.945 & $-1.6616 \mathrm{e}-07$ & 2.128 \\
\hline
\end{tabular}


TABle 69. Test: IC1 - Q4 - FS3 R2 - AS2 R2

\begin{tabular}{|r|r|rl|rr|rr|}
\hline Time steps & Cells & $e_{\mathcal{M}}(\tilde{u})$ & Rate & $\tilde{e}_{\mathcal{M}}(\tilde{u}, \tilde{\phi})$ & Rate & $a c c(\tilde{u}, \tilde{\phi})$ & Rate \\
\hline 35 & 30 & $-7.1250 \mathrm{e}-05$ & & $5.2348 \mathrm{e}-05$ & & $-1.2360 \mathrm{e}-04$ & \\
70 & 60 & $-3.4832 \mathrm{e}-06$ & 4.354 & $7.4468 \mathrm{e}-06$ & 2.813 & $-1.0930 \mathrm{e}-05$ & 3.499 \\
140 & 120 & $2.2199 \mathrm{e}-06$ & 0.650 & $1.1633 \mathrm{e}-06$ & 2.678 & $1.0566 \mathrm{e}-06$ & 3.371 \\
280 & 240 & $1.1985 \mathrm{e}-06$ & 0.889 & $2.6976 \mathrm{e}-07$ & 2.108 & $9.2871 \mathrm{e}-07$ & 0.186 \\
560 & 480 & $4.3131 \mathrm{e}-07$ & 1.474 & $8.2350 \mathrm{e}-08$ & 1.712 & $3.4897 \mathrm{e}-07$ & 1.412 \\
1120 & 960 & $1.3456 \mathrm{e}-07$ & 1.681 & $2.6059 \mathrm{e}-08$ & 1.660 & $1.0850 \mathrm{e}-07$ & 1.685 \\
\hline
\end{tabular}

4.5. Solution moment of order two. This QOI also corresponds to a discontinuous adjoint solution. The results are similar to Section 4.4, except that the error in the quantity of interest does not exhibit the superconvergence behavior with first order upwind. The accuracy of the computable error estimate is still poor when the forward solution is estimated using the TVD method. Examples are provided in Tables 70-74.

TABLE 70. Test: IC1 - Q5 - FS1 R1 - AS1 R1

\begin{tabular}{|r|r|rr|rr|rr|}
\hline Time steps & Cells & $e_{\mathcal{M}}(\tilde{u})$ & Rate & $\tilde{e}_{\mathcal{M}}(\tilde{u}, \tilde{\phi})$ & Rate & $a c c(\tilde{u}, \tilde{\phi})$ & Rate \\
\hline 35 & 30 & $-5.2225 \mathrm{e}-03$ & & $-4.7629 \mathrm{e}-03$ & & $-4.5965 \mathrm{e}-04$ & \\
70 & 60 & $-2.5034 \mathrm{e}-03$ & 1.061 & $-2.4103 \mathrm{e}-03$ & 0.983 & $-9.3150 \mathrm{e}-05$ & 2.303 \\
140 & 120 & $-1.2221 \mathrm{e}-03$ & 1.035 & $-1.2030 \mathrm{e}-03$ & 1.003 & $-1.9128 \mathrm{e}-05$ & 2.284 \\
280 & 240 & $-6.0327 \mathrm{e}-04$ & 1.018 & $-5.9910 \mathrm{e}-04$ & 1.006 & $-4.1704 \mathrm{e}-06$ & 2.197 \\
560 & 480 & $-2.9964 \mathrm{e}-04$ & 1.010 & $-2.9868 \mathrm{e}-04$ & 1.004 & $-9.6035 \mathrm{e}-07$ & 2.119 \\
1120 & 960 & $-1.4932 \mathrm{e}-04$ & 1.005 & $-1.4909 \mathrm{e}-04$ & 1.002 & $-2.2944 \mathrm{e}-07$ & 2.065 \\
\hline
\end{tabular}

TABLE 71. Test: IC1 - Q5 - FS1 R1 - AS2 R2

\begin{tabular}{|r|r|rr|rr|rr|}
\hline Time steps & Cells & $e_{\mathcal{M}}(\tilde{u})$ & Rate & $\tilde{e}_{\mathcal{M}}(\tilde{u}, \tilde{\phi})$ & Rate & $a c c(\tilde{u}, \tilde{\phi})$ & Rate \\
\hline 35 & 30 & $-5.2225 \mathrm{e}-03$ & & $-5.1856 \mathrm{e}-03$ & & $-3.6941 \mathrm{e}-05$ & \\
70 & 60 & $-2.5034 \mathrm{e}-03$ & 1.061 & $-2.5060 \mathrm{e}-03$ & 1.049 & $2.5578 \mathrm{e}-06$ & 3.852 \\
140 & 120 & $-1.2221 \mathrm{e}-03$ & 1.035 & $-1.2229 \mathrm{e}-03$ & 1.035 & $7.7334 \mathrm{e}-07$ & 1.726 \\
280 & 240 & $-6.0327 \mathrm{e}-04$ & 1.018 & $-6.0339 \mathrm{e}-04$ & 1.019 & $1.2499 \mathrm{e}-07$ & 2.629 \\
560 & 480 & $-2.9964 \mathrm{e}-04$ & 1.010 & $-2.9966 \mathrm{e}-04$ & 1.010 & $1.7391 \mathrm{e}-08$ & 2.845 \\
1120 & 960 & $-1.4932 \mathrm{e}-04$ & 1.005 & $-1.4932 \mathrm{e}-04$ & 1.005 & $2.2841 \mathrm{e}-09$ & 2.929 \\
\hline
\end{tabular}

TABLE 72. Test: IC1 - Q5 - FS2 R2 - AS2 R2

\begin{tabular}{|r|r|rr|rr|rr|}
\hline Time steps & Cells & $e_{\mathcal{M}}(\tilde{u})$ & Rate & $\tilde{e}_{\mathcal{M}}(\tilde{u}, \tilde{\phi})$ & Rate & $a c c(\tilde{u}, \tilde{\phi})$ & Rate \\
\hline 35 & 30 & $3.1553 \mathrm{e}-04$ & & $3.1761 \mathrm{e}-04$ & & $-2.0767 \mathrm{e}-06$ & \\
70 & 60 & $8.6792 \mathrm{e}-05$ & 1.862 & $8.6905 \mathrm{e}-05$ & 1.870 & $-1.1233 \mathrm{e}-07$ & 4.208 \\
140 & 120 & $2.2551 \mathrm{e}-05$ & 1.944 & $2.2557 \mathrm{e}-05$ & 1.946 & $-6.0469 \mathrm{e}-09$ & 4.215 \\
280 & 240 & $5.7358 \mathrm{e}-06$ & 1.975 & $5.7361 \mathrm{e}-06$ & 1.975 & $-3.4070 \mathrm{e}-10$ & 4.150 \\
560 & 480 & $1.4457 \mathrm{e}-06$ & 1.988 & $1.4457 \mathrm{e}-06$ & 1.988 & $-2.0020 \mathrm{e}-11$ & 4.089 \\
1120 & 960 & $3.6285 \mathrm{e}-07$ & 1.994 & $3.6285 \mathrm{e}-07$ & 1.994 & $-1.2041 \mathrm{e}-12$ & 4.055 \\
\hline
\end{tabular}


TABLE 73. Test: IC1 - Q5 - FS3 R1 - AS1 R1

\begin{tabular}{|r|r|rr|rr|rr|}
\hline Time steps & Cells & $e_{\mathcal{M}}(\tilde{u})$ & Rate & $\tilde{e}_{\mathcal{M}}(\tilde{u}, \tilde{\phi})$ & Rate & $a c c(\tilde{u}, \tilde{\phi})$ & Rate \\
\hline 35 & 30 & $-5.8340 \mathrm{e}-04$ & & $2.0174 \mathrm{e}-04$ & & $-7.8514 \mathrm{e}-04$ & \\
70 & 60 & $-1.2970 \mathrm{e}-04$ & 2.169 & $1.7422 \mathrm{e}-05$ & 3.533 & $-1.4712 \mathrm{e}-04$ & 2.416 \\
140 & 120 & $-2.9113 \mathrm{e}-05$ & 2.155 & $-2.2690 \mathrm{e}-06$ & 2.941 & $-2.6844 \mathrm{e}-05$ & 2.454 \\
280 & 240 & $-6.6101 \mathrm{e}-06$ & 2.139 & $-1.5775 \mathrm{e}-06$ & 0.524 & $-5.0326 \mathrm{e}-06$ & 2.415 \\
560 & 480 & $-1.5182 \mathrm{e}-06$ & 2.122 & $-5.2780 \mathrm{e}-07$ & 1.580 & $-9.9041 \mathrm{e}-07$ & 2.345 \\
1120 & 960 & $-3.5256 \mathrm{e}-07$ & 2.106 & $-1.4675 \mathrm{e}-07$ & 1.847 & $-2.0581 \mathrm{e}-07$ & 2.267 \\
\hline
\end{tabular}

TABle 74. Test: IC1 - Q5 - FS3 R2 - AS2 R2

\begin{tabular}{|r|r|rr|rr|rr|}
\hline Time steps & Cells & $e_{\mathcal{M}}(\tilde{u})$ & Rate & $\tilde{e}_{\mathcal{M}}(\tilde{u}, \tilde{\phi})$ & Rate & $a c c(\tilde{u}, \tilde{\phi})$ & Rate \\
\hline 35 & 30 & $-5.8304 \mathrm{e}-04$ & & $-4.5371 \mathrm{e}-04$ & & $-1.2933 \mathrm{e}-04$ & \\
70 & 60 & $-1.2967 \mathrm{e}-04$ & 2.169 & $-1.1780 \mathrm{e}-04$ & 1.945 & $-1.1874 \mathrm{e}-05$ & 3.445 \\
140 & 120 & $-2.9112 \mathrm{e}-05$ & 2.155 & $-2.9996 \mathrm{e}-05$ & 1.974 & $8.8401 \mathrm{e}-07$ & 3.748 \\
280 & 240 & $-6.6100 \mathrm{e}-06$ & 2.139 & $-7.5090 \mathrm{e}-06$ & 1.998 & $8.9895 \mathrm{e}-07$ & -0.024 \\
560 & 480 & $-1.5182 \mathrm{e}-06$ & 2.122 & $-1.8623 \mathrm{e}-06$ & 2.011 & $3.4413 \mathrm{e}-07$ & 1.385 \\
1120 & 960 & $-3.5256 \mathrm{e}-07$ & 2.106 & $-4.6030 \mathrm{e}-07$ & 2.016 & $1.0774 \mathrm{e}-07$ & 1.675 \\
\hline
\end{tabular}

4.6. Solution energy. Since this quantity of interest is nonlinear, a linearization was applied as described by (4.13)-(4.14). Clearly the additional error committed, described by (4.14), limits the accuracy of the computable error estimate to at best

$$
\mathcal{O}\left(\|u(T)-\tilde{u}(T)\|_{\Omega}^{2}\right)
$$

Unfortunately, Theorem 3.1 does not hold since the adjoint solution is defined in terms of the approximation $\tilde{u}$, which is not continuous. However, since the discontinuities of the adjoint solution in space at each discrete time occur precisely at the cell interfaces there is reason to believe the computable error estimate may still converge to the true error.

The first order upwind method is used to estimate the forward solution (smooth) in Tables 75 - 76. The former set of results shows second order accuracy of the computable error estimate. It is not surprising the same accuracy is achieved with the second order adjoint solver since the forward solver is still first order, so by the above arguments second order accuracy is the best that can be expected.

The second order upwind method was used to estimate the forward solution in Tables 77 - 78. Perhaps surprisingly, the computable error estimate has fourth order accuracy using the first order adjoint solver, and fifth order accuracy using the second order adjoint solver. An order of convergence could potentially be attributed to the superconvergence of the true error in this case. However, comparing these results with Tables 79 - 80 we see that these seemingly high convergence rates disappear when the forward solution is estimated using the TVD method. In these cases the accuracy of the computable error estimate is nominally third order, using both first and second order adjoint solvers. In this case the accuracy appears to be somehow limited by the roughness of the adjoint solution and the use of a limited finite volume solver on a smooth solution. However, in all cases it is clear that the computable error estimate is converging to the true error. 
TABLE 75. Test: IC1 - Q6 - FS1 R1 - AS1 R1

\begin{tabular}{|r|r|rr|rr|rr|}
\hline Time steps & Cells & $e_{\mathcal{M}}(\tilde{u})$ & Rate & $\tilde{e}_{\mathcal{M}}(\tilde{u}, \tilde{\phi})$ & Rate & $a c c(\tilde{u}, \tilde{\phi})$ & Rate \\
\hline 35 & 30 & $4.2811 \mathrm{e}-02$ & & $3.5949 \mathrm{e}-02$ & & $6.8620 \mathrm{e}-03$ & \\
70 & 60 & $2.2424 \mathrm{e}-02$ & 0.933 & $2.0976 \mathrm{e}-02$ & 0.777 & $1.4472 \mathrm{e}-03$ & 2.245 \\
140 & 120 & $1.1477 \mathrm{e}-02$ & 0.966 & $1.1156 \mathrm{e}-02$ & 0.911 & $3.2136 \mathrm{e}-04$ & 2.171 \\
280 & 240 & $5.8062 \mathrm{e}-03$ & 0.983 & $5.7314 \mathrm{e}-03$ & 0.961 & $7.4836 \mathrm{e}-05$ & 2.102 \\
560 & 480 & $2.9202 \mathrm{e}-03$ & 0.992 & $2.9022 \mathrm{e}-03$ & 0.982 & $1.7992 \mathrm{e}-05$ & 2.056 \\
1120 & 960 & $1.4644 \mathrm{e}-03$ & 0.996 & $1.4600 \mathrm{e}-03$ & 0.991 & $4.4067 \mathrm{e}-06$ & 2.030 \\
\hline
\end{tabular}

TABle 76. Test: IC1 - Q6 - FS1 R1 - AS2 R2

\begin{tabular}{|r|r|rr|rr|rr|}
\hline Time steps & Cells & $e_{\mathcal{M}}(\tilde{u})$ & Rate & $\tilde{e}_{\mathcal{M}}(\tilde{u}, \tilde{\phi})$ & Rate & $a c c(\tilde{u}, \tilde{\phi})$ & Rate \\
\hline 35 & 30 & $4.2811 \mathrm{e}-02$ & & $4.0735 \mathrm{e}-02$ & & $2.0759 \mathrm{e}-03$ & \\
70 & 60 & $2.2424 \mathrm{e}-02$ & 0.933 & $2.1894 \mathrm{e}-02$ & 0.896 & $5.2955 \mathrm{e}-04$ & 1.971 \\
140 & 120 & $1.1477 \mathrm{e}-02$ & 0.966 & $1.1342 \mathrm{e}-02$ & 0.949 & $1.3496 \mathrm{e}-04$ & 1.972 \\
280 & 240 & $5.8062 \mathrm{e}-03$ & 0.983 & $5.7721 \mathrm{e}-03$ & 0.975 & $3.4115 \mathrm{e}-05$ & 1.984 \\
560 & 480 & $2.9202 \mathrm{e}-03$ & 0.992 & $2.9116 \mathrm{e}-03$ & 0.987 & $8.5780 \mathrm{e}-06$ & 1.992 \\
1120 & 960 & $1.4644 \mathrm{e}-03$ & 0.996 & $1.4622 \mathrm{e}-03$ & 0.994 & $2.1507 \mathrm{e}-06$ & 1.996 \\
\hline
\end{tabular}

TABLE 77. Test: IC1 - Q6 - FS2 R2 - AS1 R1

\begin{tabular}{|r|r|rr|rr|rr|}
\hline Time steps & Cells & $e_{\mathcal{M}}(\tilde{u})$ & Rate & $\tilde{e}_{\mathcal{M}}(\tilde{u}, \tilde{\phi})$ & Rate & $a c c(\tilde{u}, \tilde{\phi})$ & Rate \\
\hline 35 & 30 & $4.6383 \mathrm{e}-04$ & & $4.4255 \mathrm{e}-04$ & & $2.1275 \mathrm{e}-05$ & \\
70 & 60 & $5.7364 \mathrm{e}-05$ & 3.015 & $5.5900 \mathrm{e}-05$ & 2.985 & $1.4643 \mathrm{e}-06$ & 3.861 \\
140 & 120 & $7.1216 \mathrm{e}-06$ & 3.010 & $7.0262 \mathrm{e}-06$ & 2.992 & $9.5441 \mathrm{e}-08$ & 3.939 \\
280 & 240 & $8.8687 \mathrm{e}-07$ & 3.005 & $8.8079 \mathrm{e}-07$ & 2.996 & $6.0821 \mathrm{e}-09$ & 3.972 \\
560 & 480 & $1.1064 \mathrm{e}-07$ & 3.003 & $1.1026 \mathrm{e}-07$ & 2.998 & $3.8371 \mathrm{e}-10$ & 3.986 \\
1120 & 960 & $1.3817 \mathrm{e}-08$ & 3.001 & $1.3792 \mathrm{e}-08$ & 2.999 & $2.4103 \mathrm{e}-11$ & 3.993 \\
\hline
\end{tabular}

TABLE 78. Test: IC1 - Q6 - FS2 R2 - AS2 R2

\begin{tabular}{|r|r|rr|rr|rr|}
\hline Time steps & Cells & $e_{\mathcal{M}}(\tilde{u})$ & Rate & $\tilde{e}_{\mathcal{M}}(\tilde{u}, \tilde{\phi})$ & Rate & $a c c(\tilde{u}, \tilde{\phi})$ & Rate \\
\hline 35 & 30 & $4.6383 \mathrm{e}-04$ & & $4.4603 \mathrm{e}-04$ & & $1.7798 \mathrm{e}-05$ & \\
70 & 60 & $5.7364 \mathrm{e}-05$ & 3.015 & $5.6809 \mathrm{e}-05$ & 2.973 & $5.5497 \mathrm{e}-07$ & 5.003 \\
140 & 120 & $7.1216 \mathrm{e}-06$ & 3.010 & $7.1044 \mathrm{e}-06$ & 2.999 & $1.7272 \mathrm{e}-08$ & 5.006 \\
280 & 240 & $8.8687 \mathrm{e}-07$ & 3.005 & $8.8633 \mathrm{e}-07$ & 3.003 & $5.3829 \mathrm{e}-10$ & 5.004 \\
560 & 480 & $1.1064 \mathrm{e}-07$ & 3.003 & $1.1063 \mathrm{e}-07$ & 3.002 & $1.6766 \mathrm{e}-11$ & 5.005 \\
1120 & 960 & $1.3817 \mathrm{e}-08$ & 3.001 & $1.3816 \mathrm{e}-08$ & 3.001 & $4.6585 \mathrm{e}-13$ & 5.170 \\
\hline
\end{tabular}


TABLE 79. Test: IC1 - Q6 - FS3 R2 - AS1 R1

\begin{tabular}{|r|r|rr|rr|rr|}
\hline Time steps & Cells & $e_{\mathcal{M}}(\tilde{u})$ & Rate & $\tilde{e}_{\mathcal{M}}(\tilde{u}, \tilde{\phi})$ & Rate & $a c c(\tilde{u}, \tilde{\phi})$ & Rate \\
\hline 35 & 30 & $3.6937 \mathrm{e}-03$ & & $3.4979 \mathrm{e}-03$ & & $1.9577 \mathrm{e}-04$ & \\
70 & 60 & $8.5363 \mathrm{e}-04$ & 2.113 & $8.3111 \mathrm{e}-04$ & 2.073 & $2.2524 \mathrm{e}-05$ & 3.120 \\
140 & 120 & $2.0445 \mathrm{e}-04$ & 2.062 & $2.0189 \mathrm{e}-04$ & 2.041 & $2.5571 \mathrm{e}-06$ & 3.139 \\
280 & 240 & $5.0006 \mathrm{e}-05$ & 2.032 & $4.9710 \mathrm{e}-05$ & 2.022 & $2.9528 \mathrm{e}-07$ & 3.114 \\
560 & 480 & $1.2367 \mathrm{e}-05$ & 2.016 & $1.2332 \mathrm{e}-05$ & 2.011 & $3.4902 \mathrm{e}-08$ & 3.081 \\
1120 & 960 & $3.0753 \mathrm{e}-06$ & 2.008 & $3.0711 \mathrm{e}-06$ & 2.006 & $4.2149 \mathrm{e}-09$ & 3.050 \\
\hline
\end{tabular}

TABLE 80. Test: IC1 - Q6 - FS3 R2 - AS2 R2

\begin{tabular}{|r|r|rr|rr|rr|}
\hline Time steps & Cells & $e_{\mathcal{M}}(\tilde{u})$ & Rate & $\tilde{e}_{\mathcal{M}}(\tilde{u}, \tilde{\phi})$ & Rate & $a c c(\tilde{u}, \tilde{\phi})$ & Rate \\
\hline 35 & 30 & $3.6937 \mathrm{e}-03$ & & $3.5462 \mathrm{e}-03$ & & $1.4749 \mathrm{e}-04$ & \\
70 & 60 & $8.5363 \mathrm{e}-04$ & 2.113 & $8.4012 \mathrm{e}-04$ & 2.078 & $1.3508 \mathrm{e}-05$ & 3.449 \\
140 & 120 & $2.0445 \mathrm{e}-04$ & 2.062 & $2.0314 \mathrm{e}-04$ & 2.048 & $1.3094 \mathrm{e}-06$ & 3.367 \\
280 & 240 & $5.0006 \mathrm{e}-05$ & 2.032 & $4.9875 \mathrm{e}-05$ & 2.026 & $1.3092 \mathrm{e}-07$ & 3.322 \\
560 & 480 & $1.2367 \mathrm{e}-05$ & 2.016 & $1.2353 \mathrm{e}-05$ & 2.013 & $1.3320 \mathrm{e}-08$ & 3.297 \\
1120 & 960 & $3.0753 \mathrm{e}-06$ & 2.008 & $3.0740 \mathrm{e}-06$ & 2.007 & $1.3663 \mathrm{e}-09$ & 3.285 \\
\hline
\end{tabular}

4.7. Solution average value over a patch. In this section the quantity of interest is the average of the solution at the final time over the patch $0.25 \leq x \leq 0.75$. Thus the adjoint solution is discontinuous and it is expected the accuracy of the computable error estimate will suffer in some cases. An alternative quantity of interest will be explored that is a $C^{1}$ approximation to $\psi_{7}(x)$ for comparison. This is accomplished by defining a cubic polynomial that transitions monotonically from 0 to 2 over the small interval $0.24<x<0.25$ and another cubic polynomial that transitions monotonically from 2 back to 0 over $0.75<x<0.76$. The value of the kernel is then set to 2 on $0.25<x<0.75$ and zero outside of $0.24<x<0.76$. The polynomials have a derivative equal to zero at the endpoints of the intervals to ensure the resulting approximation of the step function is $C^{1}$. In the tables below the results using the approximate step function are denoted by substituting $\tilde{Q} 7$ for Q7 in the labels.

The results in this section are all generated using the smooth, sinusoidal initial condition for the forward problem. In Tables 81 - 83 we see that the asymptotic behavior of the true error in the QOI and the accuracy of the computable error estimate is what would be expected for a smooth adjoint solution. Also, the results using the second order upwind forward solver, in Tables 84 - 86, resemble the results in tests using smooth solutions in Section 4.1.2. The tests using the nonlinear forward solver, in Tables 87-89, show that there is a difficulty in achieving a high accuracy of the computable error estimate with the three adjoint solvers tested.

The analogous tests using the smoothed step function for the adjoint data are shown in Tables 90 - 98. Essentially there is not any improvement in the accuracy of the computable error estimate, except in the case when using the second order upwind solver for the adjoint problem and either first order upwind or the TVD method for the forward problem. In these cases the improvement in accuracy is overshadowed by the fact that the error in approximating the step function is on 
the order of $3.8 \cdot 10^{-4}$. However, a close examination of the convergence rates of the accuracy of the computable error estimate shows that these rates are often higher using the smoothed step function compared to the exact step.

We may conclude that the failure of this approach to improving the results is due to two reasons. First, the accuracy of the computable error estimate using the exact step function is often quite good, so that no improvement can easily be made. Second, to achieve an improvement in accuracy of the computable error estimate using the approximated step function, the transition region must be adequately resolved. As this region is made smaller (thus making the exact result using the approximated step function closer to true error with the exact step function), it becomes more expensive to resolve the transitional behavior and it seems this expense makes the approach untenable, at least using uniform grid refinements. It remains to be seen if this approach would work using a special grid refinement technique.

TABle 81. Test: IC1 - Q7 - FS1 R1 - AS1 R1

\begin{tabular}{|r|r|rl|rr|rr|}
\hline Time steps & Cells & $e_{\mathcal{M}}(\tilde{u})$ & Rate & $\tilde{e}_{\mathcal{M}}(\tilde{u}, \tilde{\phi})$ & Rate & $a c c(\tilde{u}, \tilde{\phi})$ & Rate \\
\hline 35 & 30 & $6.0242 \mathrm{e}-02$ & & $5.0201 \mathrm{e}-02$ & & $1.0041 \mathrm{e}-02$ & \\
70 & 60 & $2.9221 \mathrm{e}-02$ & 1.044 & $2.8019 \mathrm{e}-02$ & 0.841 & $1.2019 \mathrm{e}-03$ & 3.062 \\
140 & 120 & $1.4785 \mathrm{e}-02$ & 0.983 & $1.4545 \mathrm{e}-02$ & 0.946 & $2.3976 \mathrm{e}-04$ & 2.326 \\
280 & 240 & $7.4361 \mathrm{e}-03$ & 0.991 & $7.3841 \mathrm{e}-03$ & 0.978 & $5.2043 \mathrm{e}-05$ & 2.204 \\
560 & 480 & $3.7290 \mathrm{e}-03$ & 0.996 & $3.7170 \mathrm{e}-03$ & 0.990 & $1.2005 \mathrm{e}-05$ & 2.116 \\
1120 & 960 & $1.8673 \mathrm{e}-03$ & 0.998 & $1.8644 \mathrm{e}-03$ & 0.995 & $2.8742 \mathrm{e}-06$ & 2.062 \\
\hline
\end{tabular}

TABLE 82. Test: IC1 - Q7 - FS1 R1 - AS2 R2

\begin{tabular}{|r|r|rc|rc|rr|}
\hline Time steps & Cells & $e_{\mathcal{M}}(\tilde{u})$ & Rate & $\tilde{e}_{\mathcal{M}}(\tilde{u}, \tilde{\phi})$ & Rate & $a c c(\tilde{u}, \tilde{\phi})$ & Rate \\
\hline 35 & 30 & $6.0242 \mathrm{e}-02$ & & $5.6881 \mathrm{e}-02$ & & $3.3612 \mathrm{e}-03$ & \\
70 & 60 & $2.9221 \mathrm{e}-02$ & 1.044 & $2.9245 \mathrm{e}-02$ & 0.960 & $-2.3770 \mathrm{e}-05$ & 7.144 \\
140 & 120 & $1.4785 \mathrm{e}-02$ & 0.983 & $1.4788 \mathrm{e}-02$ & 0.984 & $-3.2654 \mathrm{e}-06$ & 2.864 \\
280 & 240 & $7.4361 \mathrm{e}-03$ & 0.991 & $7.4365 \mathrm{e}-03$ & 0.992 & $-4.1996 \mathrm{e}-07$ & 2.959 \\
560 & 480 & $3.7290 \mathrm{e}-03$ & 0.996 & $3.7291 \mathrm{e}-03$ & 0.996 & $-5.3020 \mathrm{e}-08$ & 2.986 \\
1120 & 960 & $1.8673 \mathrm{e}-03$ & 0.998 & $1.8673 \mathrm{e}-03$ & 0.998 & $-6.6537 \mathrm{e}-09$ & 2.994 \\
\hline
\end{tabular}

TABLE 83. Test: IC1 - Q7 - FS1 R1 - AS3 R2

\begin{tabular}{|r|r|rr|rr|rr|}
\hline Time steps & Cells & $e_{\mathcal{M}}(\tilde{u})$ & Rate & $\tilde{e}_{\mathcal{M}}(\tilde{u}, \tilde{\phi})$ & Rate & $\operatorname{acc}(\tilde{u}, \tilde{\phi})$ & Rate \\
\hline 35 & 30 & $6.0242 \mathrm{e}-02$ & & $5.5731 \mathrm{e}-02$ & & $4.5108 \mathrm{e}-03$ & \\
70 & 60 & $2.9221 \mathrm{e}-02$ & 1.044 & $2.8959 \mathrm{e}-02$ & 0.944 & $2.6271 \mathrm{e}-04$ & 4.102 \\
140 & 120 & $1.4785 \mathrm{e}-02$ & 0.983 & $1.4728 \mathrm{e}-02$ & 0.975 & $5.6609 \mathrm{e}-05$ & 2.214 \\
280 & 240 & $7.4361 \mathrm{e}-03$ & 0.991 & $7.4242 \mathrm{e}-03$ & 0.988 & $1.1913 \mathrm{e}-05$ & 2.248 \\
560 & 480 & $3.7290 \mathrm{e}-03$ & 0.996 & $3.7265 \mathrm{e}-03$ & 0.994 & $2.4655 \mathrm{e}-06$ & 2.273 \\
1120 & 960 & $1.8673 \mathrm{e}-03$ & 0.998 & $1.8667 \mathrm{e}-03$ & 0.997 & $5.0463 \mathrm{e}-07$ & 2.289 \\
\hline
\end{tabular}


TABLE 84. Test: IC1 - Q7 - FS2 R2 - AS1 R1

\begin{tabular}{|r|r|rc|rc|rr|}
\hline Time steps & Cells & $e_{\mathcal{M}}(\tilde{u})$ & Rate & $\tilde{e}_{\mathcal{M}}(\tilde{u}, \tilde{\phi})$ & Rate & $a c c(\tilde{u}, \tilde{\phi})$ & Rate \\
\hline 35 & 30 & $4.0583 \mathrm{e}-03$ & & $5.6566 \mathrm{e}-04$ & & $3.4926 \mathrm{e}-03$ & \\
70 & 60 & $7.1983 \mathrm{e}-05$ & 5.817 & $7.1450 \mathrm{e}-05$ & 2.985 & $5.3266 \mathrm{e}-07$ & 12.679 \\
140 & 120 & $9.0014 \mathrm{e}-06$ & 2.999 & $8.9616 \mathrm{e}-06$ & 2.995 & $3.9813 \mathrm{e}-08$ & 3.742 \\
280 & 240 & $1.1251 \mathrm{e}-06$ & 3.000 & $1.1224 \mathrm{e}-06$ & 2.997 & $2.6909 \mathrm{e}-09$ & 3.887 \\
560 & 480 & $1.4062 \mathrm{e}-07$ & 3.000 & $1.4044 \mathrm{e}-07$ & 2.999 & $1.7450 \mathrm{e}-10$ & 3.947 \\
1120 & 960 & $1.7576 \mathrm{e}-08$ & 3.000 & $1.7564 \mathrm{e}-08$ & 2.999 & $1.1115 \mathrm{e}-11$ & 3.973 \\
\hline
\end{tabular}

TABle 85. Test: IC1 - Q7 - FS2 R2 - AS2 R2

\begin{tabular}{|r|r|rr|rr|rr|}
\hline Time steps & Cells & $e_{\mathcal{M}}(\tilde{u})$ & Rate & $\tilde{e}_{\mathcal{M}}(\tilde{u}, \tilde{\phi})$ & Rate & $a c c(\tilde{u}, \tilde{\phi})$ & Rate \\
\hline 35 & 30 & $4.0583 \mathrm{e}-03$ & & $5.7067 \mathrm{e}-04$ & & $3.4876 \mathrm{e}-03$ & \\
70 & 60 & $7.1983 \mathrm{e}-05$ & 5.817 & $7.2621 \mathrm{e}-05$ & 2.974 & $-6.3853 \mathrm{e}-07$ & 12.415 \\
140 & 120 & $9.0014 \mathrm{e}-06$ & 2.999 & $9.0614 \mathrm{e}-06$ & 3.003 & $-6.0011 \mathrm{e}-08$ & 3.411 \\
280 & 240 & $1.1251 \mathrm{e}-06$ & 3.000 & $1.1294 \mathrm{e}-06$ & 3.004 & $-4.3749 \mathrm{e}-09$ & 3.778 \\
560 & 480 & $1.4062 \mathrm{e}-07$ & 3.000 & $1.4091 \mathrm{e}-07$ & 3.003 & $-2.9290 \mathrm{e}-10$ & 3.901 \\
1120 & 960 & $1.7576 \mathrm{e}-08$ & 3.000 & $1.7595 \mathrm{e}-08$ & 3.002 & $-1.8971 \mathrm{e}-11$ & 3.949 \\
\hline
\end{tabular}

TABLE 86. Test: IC1 - Q7 - FS2 R2 - AS3 R2

\begin{tabular}{|r|r|rr|rr|rr|}
\hline Time steps & Cells & $e_{\mathcal{M}}(\tilde{u})$ & Rate & $\tilde{e}_{\mathcal{M}}(\tilde{u}, \tilde{\phi})$ & Rate & $a c c(\tilde{u}, \tilde{\phi})$ & Rate \\
\hline 35 & 30 & $4.0583 \mathrm{e}-03$ & & $5.5658 \mathrm{e}-04$ & & $3.5017 \mathrm{e}-03$ & \\
70 & 60 & $7.1983 \mathrm{e}-05$ & 5.817 & $7.1772 \mathrm{e}-05$ & 2.955 & $2.1065 \mathrm{e}-07$ & 14.021 \\
140 & 120 & $9.0014 \mathrm{e}-06$ & 2.999 & $9.0175 \mathrm{e}-06$ & 2.993 & $-1.6133 \mathrm{e}-08$ & 3.707 \\
280 & 240 & $1.1251 \mathrm{e}-06$ & 3.000 & $1.1272 \mathrm{e}-06$ & 3.000 & $-2.1182 \mathrm{e}-09$ & 2.929 \\
560 & 480 & $1.4062 \mathrm{e}-07$ & 3.000 & $1.4079 \mathrm{e}-07$ & 3.001 & $-1.7687 \mathrm{e}-10$ & 3.582 \\
1120 & 960 & $1.7576 \mathrm{e}-08$ & 3.000 & $1.7589 \mathrm{e}-08$ & 3.001 & $-1.3002 \mathrm{e}-11$ & 3.766 \\
\hline
\end{tabular}

TABLE 87. Test: IC1 - Q7 - FS3 R2 - AS1 R1

\begin{tabular}{|r|r|rl|rr|rr|}
\hline Time steps & Cells & $e_{\mathcal{M}}(\tilde{u})$ & Rate & $\tilde{e}_{\mathcal{M}}(\tilde{u}, \tilde{\phi})$ & Rate & $a c c(\tilde{u}, \tilde{\phi})$ & Rate \\
\hline 35 & 30 & $5.1898 \mathrm{e}-03$ & & $2.4244 \mathrm{e}-03$ & & $2.7654 \mathrm{e}-03$ & \\
70 & 60 & $2.3752 \mathrm{e}-04$ & 4.450 & $3.7720 \mathrm{e}-04$ & 2.684 & $-1.3967 \mathrm{e}-04$ & 4.307 \\
140 & 120 & $3.5230 \mathrm{e}-05$ & 2.753 & $6.2353 \mathrm{e}-05$ & 2.597 & $-2.7122 \mathrm{e}-05$ & 2.364 \\
280 & 240 & $5.2916 \mathrm{e}-06$ & 2.735 & $1.0382 \mathrm{e}-05$ & 2.586 & $-5.0900 \mathrm{e}-06$ & 2.414 \\
560 & 480 & $8.0323 \mathrm{e}-07$ & 2.720 & $1.7466 \mathrm{e}-06$ & 2.571 & $-9.4341 \mathrm{e}-07$ & 2.432 \\
1120 & 960 & $1.2282 \mathrm{e}-07$ & 2.709 & $2.9640 \mathrm{e}-07$ & 2.559 & $-1.7358 \mathrm{e}-07$ & 2.442 \\
\hline
\end{tabular}


TABle 88. Test: IC1 - Q7 - FS3 R2 - AS2 R2

\begin{tabular}{|r|r|rr|rr|rr|}
\hline Time steps & Cells & $e_{\mathcal{M}}(\tilde{u})$ & Rate & $\tilde{e}_{\mathcal{M}}(\tilde{u}, \tilde{\phi})$ & Rate & $a c c(\tilde{u}, \tilde{\phi})$ & Rate \\
\hline 35 & 30 & $5.1898 \mathrm{e}-03$ & & $1.4789 \mathrm{e}-03$ & & $3.7108 \mathrm{e}-03$ & \\
70 & 60 & $2.3752 \mathrm{e}-04$ & 4.450 & $2.3437 \mathrm{e}-04$ & 2.658 & $3.1536 \mathrm{e}-06$ & 10.201 \\
140 & 120 & $3.5230 \mathrm{e}-05$ & 2.753 & $3.4941 \mathrm{e}-05$ & 2.746 & $2.8870 \mathrm{e}-07$ & 3.449 \\
280 & 240 & $5.2916 \mathrm{e}-06$ & 2.735 & $5.3806 \mathrm{e}-06$ & 2.699 & $-8.9063 \mathrm{e}-08$ & 1.697 \\
560 & 480 & $8.0323 \mathrm{e}-07$ & 2.720 & $8.3412 \mathrm{e}-07$ & 2.689 & $-3.0891 \mathrm{e}-08$ & 1.528 \\
1120 & 960 & $1.2282 \mathrm{e}-07$ & 2.709 & $1.2933 \mathrm{e}-07$ & 2.689 & $-6.5069 \mathrm{e}-09$ & 2.247 \\
\hline
\end{tabular}

TABle 89. Test: IC1 - Q7 - FS3 R2 - AS3 R2

\begin{tabular}{|r|r|rc|rc|rr|}
\hline Time steps & Cells & $e_{\mathcal{M}}(\tilde{u})$ & Rate & $\tilde{e}_{\mathcal{M}}(\tilde{u}, \tilde{\phi})$ & Rate & $a c c(\tilde{u}, \tilde{\phi})$ & Rate \\
\hline 35 & 30 & $5.1898 \mathrm{e}-03$ & & $1.7221 \mathrm{e}-03$ & & $3.4677 \mathrm{e}-03$ & \\
70 & 60 & $2.3752 \mathrm{e}-04$ & 4.450 & $2.8199 \mathrm{e}-04$ & 2.610 & $-4.4465 \mathrm{e}-05$ & 6.285 \\
140 & 120 & $3.5230 \mathrm{e}-05$ & 2.753 & $4.3768 \mathrm{e}-05$ & 2.688 & $-8.5376 \mathrm{e}-06$ & 2.381 \\
280 & 240 & $5.2916 \mathrm{e}-06$ & 2.735 & $6.8833 \mathrm{e}-06$ & 2.669 & $-1.5917 \mathrm{e}-06$ & 2.423 \\
560 & 480 & $8.0323 \mathrm{e}-07$ & 2.720 & $1.0849 \mathrm{e}-06$ & 2.665 & $-2.8170 \mathrm{e}-07$ & 2.498 \\
1120 & 960 & $1.2282 \mathrm{e}-07$ & 2.709 & $1.7078 \mathrm{e}-07$ & 2.667 & $-4.7960 \mathrm{e}-08$ & 2.554 \\
\hline
\end{tabular}

TABLE 90. Test: IC1 - $\tilde{Q} 7$ - FS1 R1 - AS1 R1

\begin{tabular}{|r|r|rr|rr|rr|}
\hline Time steps & Cells & $e_{\mathcal{M}}(\tilde{u})$ & Rate & $\tilde{e}_{\mathcal{M}}(\tilde{u}, \tilde{\phi})$ & Rate & $a c c(\tilde{u}, \tilde{\phi})$ & Rate \\
\hline 35 & 30 & $5.7057 \mathrm{e}-02$ & & $5.0201 \mathrm{e}-02$ & & $6.8562 \mathrm{e}-03$ & \\
70 & 60 & $2.9205 \mathrm{e}-02$ & 0.966 & $2.7973 \mathrm{e}-02$ & 0.844 & $1.2319 \mathrm{e}-03$ & 2.477 \\
140 & 120 & $1.4776 \mathrm{e}-02$ & 0.983 & $1.4533 \mathrm{e}-02$ & 0.945 & $2.4324 \mathrm{e}-04$ & 2.340 \\
280 & 240 & $7.4317 \mathrm{e}-03$ & 0.991 & $7.3793 \mathrm{e}-03$ & 0.978 & $5.2444 \mathrm{e}-05$ & 2.214 \\
560 & 480 & $3.7268 \mathrm{e}-03$ & 0.996 & $3.7148 \mathrm{e}-03$ & 0.990 & $1.2051 \mathrm{e}-05$ & 2.122 \\
1120 & 960 & $1.8661 \mathrm{e}-03$ & 0.998 & $1.8633 \mathrm{e}-03$ & 0.995 & $2.8791 \mathrm{e}-06$ & 2.065 \\
\hline
\end{tabular}

TABle 91. Test: IC1 - $\tilde{Q} 7$ - FS1 R1 - AS2 R2

\begin{tabular}{|r|r|rl|rr|rr|}
\hline Time steps & Cells & $e_{\mathcal{M}}(\tilde{u})$ & Rate & $\tilde{e}_{\mathcal{M}}(\tilde{u}, \tilde{\phi})$ & Rate & $\operatorname{acc}(\tilde{u}, \tilde{\phi})$ & Rate \\
\hline 35 & 30 & $5.7057 \mathrm{e}-02$ & & $5.6881 \mathrm{e}-02$ & & $1.7660 \mathrm{e}-04$ & \\
70 & 60 & $2.9205 \mathrm{e}-02$ & 0.966 & $2.9197 \mathrm{e}-02$ & 0.962 & $8.2511 \mathrm{e}-06$ & 4.420 \\
140 & 120 & $1.4776 \mathrm{e}-02$ & 0.983 & $1.4776 \mathrm{e}-02$ & 0.983 & $4.2044 \mathrm{e}-07$ & 4.295 \\
280 & 240 & $7.4317 \mathrm{e}-03$ & 0.991 & $7.4317 \mathrm{e}-03$ & 0.991 & $1.5360 \mathrm{e}-08$ & 4.775 \\
560 & 480 & $3.7268 \mathrm{e}-03$ & 0.996 & $3.7268 \mathrm{e}-03$ & 0.996 & $8.8711 \mathrm{e}-10$ & 4.114 \\
1120 & 960 & $1.8661 \mathrm{e}-03$ & 0.998 & $1.8661 \mathrm{e}-03$ & 0.998 & $-3.2131 \mathrm{e}-11$ & 4.787 \\
\hline
\end{tabular}


TABle 92. Test: IC1 - $\tilde{Q} 7$ - FS1 R1 - AS3 R2

\begin{tabular}{|r|r|rr|rr|rr|}
\hline Time steps & Cells & $e_{\mathcal{M}}(\tilde{u})$ & Rate & $\tilde{e}_{\mathcal{M}}(\tilde{u}, \tilde{\phi})$ & Rate & $a c c(\tilde{u}, \tilde{\phi})$ & Rate \\
\hline 35 & 30 & $5.7057 \mathrm{e}-02$ & & $5.5708 \mathrm{e}-02$ & & $1.3491 \mathrm{e}-03$ & \\
70 & 60 & $2.9205 \mathrm{e}-02$ & 0.966 & $2.8931 \mathrm{e}-02$ & 0.945 & $2.7400 \mathrm{e}-04$ & 2.300 \\
140 & 120 & $1.4776 \mathrm{e}-02$ & 0.983 & $1.4719 \mathrm{e}-02$ & 0.975 & $5.6800 \mathrm{e}-05$ & 2.270 \\
280 & 240 & $7.4317 \mathrm{e}-03$ & 0.991 & $7.4202 \mathrm{e}-03$ & 0.988 & $1.1552 \mathrm{e}-05$ & 2.298 \\
560 & 480 & $3.7268 \mathrm{e}-03$ & 0.996 & $3.7246 \mathrm{e}-03$ & 0.994 & $2.2411 \mathrm{e}-06$ & 2.366 \\
1120 & 960 & $1.8661 \mathrm{e}-03$ & 0.998 & $1.8657 \mathrm{e}-03$ & 0.997 & $3.9960 \mathrm{e}-07$ & 2.488 \\
\hline
\end{tabular}

TABLE 93. Test: IC1 - $\tilde{Q} 7$ - FS2 R2 - AS1 R1

\begin{tabular}{|r|r|rc|rr|rr|}
\hline Time steps & Cells & $e_{\mathcal{M}}(\tilde{u})$ & Rate & $\tilde{e}_{\mathcal{M}}(\tilde{u}, \tilde{\phi})$ & Rate & $a c c(\tilde{u}, \tilde{\phi})$ & Rate \\
\hline 35 & 30 & $5.9847 \mathrm{e}-04$ & & $5.6566 \mathrm{e}-04$ & & $3.2808 \mathrm{e}-05$ & \\
70 & 60 & $7.3022 \mathrm{e}-05$ & 3.035 & $7.1333 \mathrm{e}-05$ & 2.987 & $1.6888 \mathrm{e}-06$ & 4.280 \\
140 & 120 & $9.0703 \mathrm{e}-06$ & 3.009 & $8.9541 \mathrm{e}-06$ & 2.994 & $1.1628 \mathrm{e}-07$ & 3.860 \\
280 & 240 & $1.1332 \mathrm{e}-06$ & 3.001 & $1.1216 \mathrm{e}-06$ & 2.997 & $1.1552 \mathrm{e}-08$ & 3.331 \\
560 & 480 & $1.4087 \mathrm{e}-07$ & 3.008 & $1.4036 \mathrm{e}-07$ & 2.998 & $5.0970 \mathrm{e}-10$ & 4.502 \\
1120 & 960 & $1.7517 \mathrm{e}-08$ & 3.007 & $1.7554 \mathrm{e}-08$ & 2.999 & $-3.6883 \mathrm{e}-11$ & 3.789 \\
\hline
\end{tabular}

TABLE 94. Test: IC1 - $\tilde{Q} 7$ - FS2 R2 - AS2 R2

\begin{tabular}{|r|r|rr|rr|rr|}
\hline Time steps & Cells & $e_{\mathcal{M}}(\tilde{u})$ & Rate & $\tilde{e}_{\mathcal{M}}(\tilde{u}, \tilde{\phi})$ & Rate & $a c c(\tilde{u}, \tilde{\phi})$ & Rate \\
\hline 35 & 30 & $5.9847 \mathrm{e}-04$ & & $5.7067 \mathrm{e}-04$ & & $2.7804 \mathrm{e}-05$ & \\
70 & 60 & $7.3022 \mathrm{e}-05$ & 3.035 & $7.2502 \mathrm{e}-05$ & 2.977 & $5.1956 \mathrm{e}-07$ & 5.742 \\
140 & 120 & $9.0703 \mathrm{e}-06$ & 3.009 & $9.0538 \mathrm{e}-06$ & 3.001 & $1.6536 \mathrm{e}-08$ & 4.974 \\
280 & 240 & $1.1332 \mathrm{e}-06$ & 3.001 & $1.1287 \mathrm{e}-06$ & 3.004 & $4.4908 \mathrm{e}-09$ & 1.881 \\
560 & 480 & $1.4087 \mathrm{e}-07$ & 3.008 & $1.4082 \mathrm{e}-07$ & 3.003 & $4.2591 \mathrm{e}-11$ & 6.720 \\
1120 & 960 & $1.7517 \mathrm{e}-08$ & 3.007 & $1.7584 \mathrm{e}-08$ & 3.002 & $-6.6952 \mathrm{e}-11$ & -0.653 \\
\hline
\end{tabular}

TABLE 95. Test: IC1 - $\tilde{Q} 7$ - FS2 R2 - AS3 R2

\begin{tabular}{|r|r|rl|rr|rr|}
\hline Time steps & Cells & $e_{\mathcal{M}}(\tilde{u})$ & Rate & $\tilde{e}_{\mathcal{M}}(\tilde{u}, \tilde{\phi})$ & Rate & $a c c(\tilde{u}, \tilde{\phi})$ & Rate \\
\hline 35 & 30 & $5.9847 \mathrm{e}-04$ & & $5.5634 \mathrm{e}-04$ & & $4.2135 \mathrm{e}-05$ & \\
70 & 60 & $7.3022 \mathrm{e}-05$ & 3.035 & $7.1709 \mathrm{e}-05$ & 2.956 & $1.3129 \mathrm{e}-06$ & 5.004 \\
140 & 120 & $9.0703 \mathrm{e}-06$ & 3.009 & $9.0122 \mathrm{e}-06$ & 2.992 & $5.8161 \mathrm{e}-08$ & 4.497 \\
280 & 240 & $1.1332 \mathrm{e}-06$ & 3.001 & $1.1266 \mathrm{e}-06$ & 3.000 & $6.6172 \mathrm{e}-09$ & 3.136 \\
560 & 480 & $1.4087 \mathrm{e}-07$ & 3.008 & $1.4072 \mathrm{e}-07$ & 3.001 & $1.4711 \mathrm{e}-10$ & 5.491 \\
1120 & 960 & $1.7517 \mathrm{e}-08$ & 3.007 & $1.7579 \mathrm{e}-08$ & 3.001 & $-6.2178 \mathrm{e}-11$ & 1.242 \\
\hline
\end{tabular}


TABLE 96. Test: IC1 - Q̃7 - FS3 R2 - AS1 R1

\begin{tabular}{|r|r|rr|rr|rr|}
\hline Time steps & Cells & $e_{\mathcal{M}}(\tilde{u})$ & Rate & $\tilde{e}_{\mathcal{M}}(\tilde{u}, \tilde{\phi})$ & Rate & $a c c(\tilde{u}, \tilde{\phi})$ & Rate \\
\hline 35 & 30 & $1.6222 \mathrm{e}-03$ & & $2.4244 \mathrm{e}-03$ & & $-8.0217 \mathrm{e}-04$ & \\
70 & 60 & $2.4562 \mathrm{e}-04$ & 2.723 & $3.8487 \mathrm{e}-04$ & 2.655 & $-1.3925 \mathrm{e}-04$ & 2.526 \\
140 & 120 & $3.7873 \mathrm{e}-05$ & 2.697 & $6.4018 \mathrm{e}-05$ & 2.588 & $-2.6146 \mathrm{e}-05$ & 2.413 \\
280 & 240 & $6.2361 \mathrm{e}-06$ & 2.602 & $1.0855 \mathrm{e}-05$ & 2.560 & $-4.6193 \mathrm{e}-06$ & 2.501 \\
560 & 480 & $1.1323 \mathrm{e}-06$ & 2.461 & $1.8990 \mathrm{e}-06$ & 2.515 & $-7.6670 \mathrm{e}-07$ & 2.591 \\
1120 & 960 & $2.3084 \mathrm{e}-07$ & 2.294 & $3.4806 \mathrm{e}-07$ & 2.448 & $-1.1722 \mathrm{e}-07$ & 2.709 \\
\hline
\end{tabular}

TABLE 97. Test: IC1 - $\tilde{Q} 7$ - FS3 R2 - AS2 R2

\begin{tabular}{|r|r|rr|rr|rr|}
\hline Time steps & Cells & $e_{\mathcal{M}}(\tilde{u})$ & Rate & $\tilde{e}_{\mathcal{M}}(\tilde{u}, \tilde{\phi})$ & Rate & $a c c(\tilde{u}, \tilde{\phi})$ & Rate \\
\hline 35 & 30 & $1.6222 \mathrm{e}-03$ & & $1.4789 \mathrm{e}-03$ & & $1.4329 \mathrm{e}-04$ & \\
70 & 60 & $2.4562 \mathrm{e}-04$ & 2.723 & $2.5115 \mathrm{e}-04$ & 2.558 & $-5.5294 \mathrm{e}-06$ & 4.696 \\
140 & 120 & $3.7873 \mathrm{e}-05$ & 2.697 & $3.9053 \mathrm{e}-05$ & 2.685 & $-1.1801 \mathrm{e}-06$ & 2.228 \\
280 & 240 & $6.2361 \mathrm{e}-06$ & 2.602 & $6.4481 \mathrm{e}-06$ & 2.598 & $-2.1193 \mathrm{e}-07$ & 2.477 \\
560 & 480 & $1.1323 \mathrm{e}-06$ & 2.461 & $1.1477 \mathrm{e}-06$ & 2.490 & $-1.5431 \mathrm{e}-08$ & 3.780 \\
1120 & 960 & $2.3084 \mathrm{e}-07$ & 2.294 & $2.3001 \mathrm{e}-07$ & 2.319 & $8.2722 \mathrm{e}-10$ & 4.221 \\
\hline
\end{tabular}

TABLE 98. Test: IC1 - $\tilde{Q} 7$ - FS3 R2 - AS3 R2

\begin{tabular}{|r|r|rc|rc|rr|}
\hline Time steps & Cells & $e_{\mathcal{M}}(\tilde{u})$ & Rate & $\tilde{e}_{\mathcal{M}}(\tilde{u}, \tilde{\phi})$ & Rate & $\operatorname{acc}(\tilde{u}, \tilde{\phi})$ & Rate \\
\hline 35 & 30 & $1.6222 \mathrm{e}-03$ & & $1.7400 \mathrm{e}-03$ & & $-1.1781 \mathrm{e}-04$ & \\
70 & 60 & $2.4562 \mathrm{e}-04$ & 2.723 & $2.9345 \mathrm{e}-04$ & 2.568 & $-4.7830 \mathrm{e}-05$ & 1.300 \\
140 & 120 & $3.7873 \mathrm{e}-05$ & 2.697 & $4.7192 \mathrm{e}-05$ & 2.636 & $-9.3192 \mathrm{e}-06$ & 2.360 \\
280 & 240 & $6.2361 \mathrm{e}-06$ & 2.602 & $7.8519 \mathrm{e}-06$ & 2.587 & $-1.6158 \mathrm{e}-06$ & 2.528 \\
560 & 480 & $1.1323 \mathrm{e}-06$ & 2.461 & $1.3411 \mathrm{e}-06$ & 2.550 & $-2.0882 \mathrm{e}-07$ & 2.952 \\
1120 & 960 & $2.3084 \mathrm{e}-07$ & 2.294 & $2.5059 \mathrm{e}-07$ & 2.420 & $-1.9753 \mathrm{e}-08$ & 3.402 \\
\hline
\end{tabular}

\section{Conclusions And Future WORK}

Numerous tests support the claims of Theorem 3.1, i.e. the rate of convergence of the computable error estimate to the true error in a quantity of interest may be predicted to some minimum order when the solution to the linear advection problem 3.1 and the associated adjoint problem (3.11) have smooth solutions. The results also show the theory to be overly pessimistic in many cases. However, the theory shows that if the goal is to reliably estimate the error in quantities of interest, there are only two cases to consider when the solutions are smooth.

The first case is when the true error in a quantity of interest converges at the standard rate associated with the forward solver method. In this case only a first order accurate adjoint solver is required to guarantee that the effectivity index (Section 4.1.2) converges to 1 . This is desirable in the absence of a computable upper bound for the error.

The second case is due to the fact that superconvergence is sometimes observed for the true error. This needs to be accounted for when estimating the adjoint 
variable, since the error cancellation does not necessarily carry through to improve the accuracy of the computable error estimate. Therefore a higher order adjoint solver may be needed to guarantee that the effectivity index converges to 1 in this second case.

The numerical tests suggest that when the forward solver involves limiters, the superconvergence property for the true error does not occur. In this case a first order adjoint solver is sufficient (again, with smooth solutions) to guarantee the accuracy of the computable error estimate is higher order than the convergence rate of the true error. Thus if the theory were extended to accomodate shocks, it is likely that the superconvergence issue would not impact real applications, where limited schemes would be used.

The theory does not hold when discontinuities are present in either the forward or adjoint solutions. Indeed, the derivation of the computable error estimate does not make sense in this case. The computations show that the adjoint error estimation technique in this report will not be reliable in this context until the theory is extended, and in particular a computable error estimate is derived with guaranteed asymptotic properties in the presence of discontinuities. In practice, more sophisticated methods of reconstruction may be necessary than those presented in this report to properly treat shocks. A recent example of such an idea is provided in [23].

We have studied an approach to calculate the average of the solution over a patch using a discontinuous step function and a smoother approximation of the step function, for which some asymptotic results should hold. The results have shown that the improvement in the accuracy of the computable error estimate using the approximate step function is outweighed by the error committed in making the approximation of the step function. The sharp transition was still not adequately resolved and generally the error using the exact step function was reasonably small in the first place, though the accuracy of the error estimate was not always very good. This problem may be more clearly resolved using some adaptive mesh routine.

It would be useful to characterize precisely when the ratio of the computable error estimate to the true error converges to 1 , which cannot currently be predicted. It is possible this type of result could only be proved for certain quantities of interest. For example, if the quantity of interest is evaluation at a point, there is no reason to believe there is a relationship between this local error and the global error that determines the accuracy of the computable error estimate. Thus the ratio of the two could behave unpredictably. However, a more likely quantity of interest for applications like an average over a patch or Fourier coefficient may exhibit a convergence rate approximately the same as for the global error in the forward solution. It seems more likely this will not be possible without developing a different computable estimate of the error, in particular one that bounds the error above.

\section{REFERENCES}

[1] Ainsworth, M. and Oden, J. T., A posteriori error estimation in finite element analysis, Comput. Methods Appl. Mech. Engrg., Vol. 142, 1997, pp. 1-88.

[2] Atkinson, K. E., An Introduction to Numerical Analysis, Second edition, John Wiley and Sons, 1988.

[3] Barter, G.E., Shock capturing with PDE-based artificial viscosity for an adaptive, higherorder discontinuous Galerkin finite element method, PhD Thesis at MIT, 2008. 
[4] Barth, T.J., A posteriori error estimation and mesh adaptivity for finite volume and finite element methods, Adaptive Mesh Refinement-Theory and Applications, Lecture Notes in CSE, Vol. 41, part II, 2005, pp. 183-202.

[5] Barth, T.J., Space-Time Error Representation and Estimation in Navier-Stokes Calculations, Complex Effects in LES, Vol. 56, 2007, pp. 29-48.

[6] Barth, T. J. and Larson, M. G., A Posteriori Error Estimates for Higher Order Godunov Finite Volume Methods on Unstructured Meshes, NASA Tech. Rept. NAS-02-001, 2002.

[7] Bensow, R.E. and Larson, M.G., Residual based VMS subgrid modeling for vortex flows, Computer Methods in Applied Mech. and Engr., Vol. 199, No 13-16, 2010, pp. 802-809.

[8] Cockburn, B., Lin, S. -Y. and Shu, C. -W., TVB Runge-Kutta Local Projection Discontinuous Galerkin Finite Element Method for Conservation Laws III: One Dimensional Systems, Jour. of Comp. Phys., Vol. 84, No. 1, 1989, pp. 90-113.

[9] Cockburn, B. and Shu, C. -W., The Runge-Kutta Discontinuous Galerkin Method for Conservation Laws V: Multidimensional Systems, Jour. of Comp. Phys., Vol. 141, No. 2, 1998, pp. 199-224.

[10] Estep, D., Pernice, M., Pham, D., Tavener, S. and Wang, H., A posteriori error analysis of a cell-centered finite volume method for semilinear elliptic problems, Journal of Computational and Applied Mathematics, Vol. 233, No. 2, 2009, pp. 459-472.

[11] Fidkowski, K.J. and Darmofal, D.L., A triangular cut-cell adaptive method for high-order discretizations of the compressible Navier-Stokes equations, JCP, Vol. 225, No. 2, 2007, pp. 1653-1672.

[12] Fidkowski, K.J. and Darmofal, D.L., Output-based error estimation and mesh adaptation in computational fluid dynamics: overview and recent results, Proceedings of the 47th AIAA Aerospace Sciences Meeting, 2009.

[13] Fidkowski, K.J. and Roe, P.L., An entropy adjoint approach to mesh refinement, SIAM J. Sci. Comp., 2010.

[14] Giles, M. B. and Süli, E., Adjoint methods for PDEs: a posteriori error analysis and postprocessing by duality, Acta Numerica, 2002, pp. 145-236.

[15] Hartmann, R., Multitarget error estimation and adaptivity in aerodynamic flow simulations, SIAM J. Sci. Comp., 2008.

[16] Hartmann, R. and Houston, P., Adaptive discontinuous Galerkin finite element methods for the compressible Euler equations, JCP, Vol. 183, No. 2, 2002, pp. 508-532.

[17] Hartmann, R. and Houston, P., Adaptive discontinuous Galerkin finite element methods for nonlinear hyperbolic conservation laws, SIAM Jour. Sci. Comp., Vol. 24, No. 3, 2002, pp. 979-1004.

[18] Marchuk, G. I., Agoshkov, V. I. and Shutyaev, V. P., Adjoint Equations and Perturbation Algorithms, CRC Press, USA, 1996.

[19] Oberkampf, W.L., Trucano, T.G. and Hirsch, C., Verification, validation and predictive capability in computational engineering and physics, Appl. Mech. Rev., Vol. 57, No. 5, 2004.

[20] Roy, C.J., Review of code and solution verification procedures for computational simulation, JCP, Vol. 205, No. 1, 2005, pp. 131-156.

[21] Shu, C. -W., Essentially non-oscillatory and weighted essentially non-oscillatory schemes for hyperbolic conservation laws, in Advanced Numerical Approximation of Nonlinear Hyperbolic Equations, Lecture Notes in Mathematics, Vol. 1697/1998, 1998, pp. 325-432.

[22] Steiner, C. and Noelle, S., On adaptive timestepping for weakly instationary solutions of hyperbolic conservation laws via adjoint error control, International Journal for Numerical Methods in Biomedical Engineering, Vol. 26, No. 6, 2010.

[23] Zhang, X. and Shu, C. -W., On maximum-principle-satisfying high order schemes for scalar conservation laws, Journal of Computational Physics, Vol. 229, 2010, pp. 3091-3120. 
(J. Connors) Lawrence Livermore National Laboratory, Center for Applied Scientific Computing, Livermore, CA, 94551, USA., This work was Performed under the auspices of the U.S. Department of Energy by Lawrence Livermore National Laboratory unDer Contract DE-AC52-07NA27344 and was Funded By the UnCERTAinty Quantification Strategic Initiative Laboratory Directed Research and Development Project at LLNL UNDER PROJECT TRACKING CODE 10-SI-013. LLNL-TR-477651.

E-mail address, J. Connors: connors4@llnl.gov

(J. Banks) Lawrence Livermore National Laboratory, Center for Applied Scientific Computing, Livermore, CA, 94551, USA.

E-mail address, J. Banks: banks20@1lnl.gov

(J. Hittinger) Lawrence Livermore National Laboratory, Center for Applied ScienTific Computing, Livermore, CA, 94551, USA.

E-mail address, J. Hittinger: hittinger1@llnl.gov

(C. Woodward) Lawrence Livermore National Laboratory, Center for Applied Scientific Computing, Livermore, CA, 94551, USA.

E-mail address, C. Woodward: woodward6@1lnl.gov 